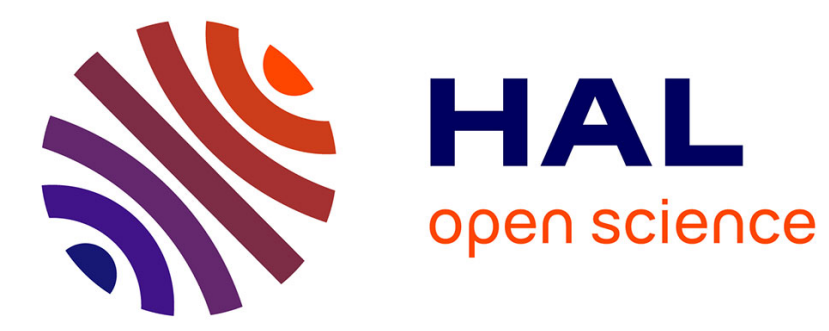

\title{
Hydrodynamic forces on a clean spherical bubble translating in a wall-bounded linear shear flow
}

\author{
Pengyu Shi, Roland Rzehak, Dirk Lucas, Jacques Magnaudet
}

\section{To cite this version:}

Pengyu Shi, Roland Rzehak, Dirk Lucas, Jacques Magnaudet. Hydrodynamic forces on a clean spherical bubble translating in a wall-bounded linear shear flow. Physical Review Fluids, 2020, 5 (7), pp.073601. 10.1103/PhysRevFluids.5.073601 . hal-02933780

\section{HAL Id: hal-02933780 \\ https://hal.science/hal-02933780}

Submitted on 8 Sep 2020

HAL is a multi-disciplinary open access archive for the deposit and dissemination of scientific research documents, whether they are published or not. The documents may come from teaching and research institutions in France or abroad, or from public or private research centers.
L'archive ouverte pluridisciplinaire HAL, est destinée au dépôt et à la diffusion de documents scientifiques de niveau recherche, publiés ou non, émanant des établissements d'enseignement et de recherche français ou étrangers, des laboratoires publics ou privés. 


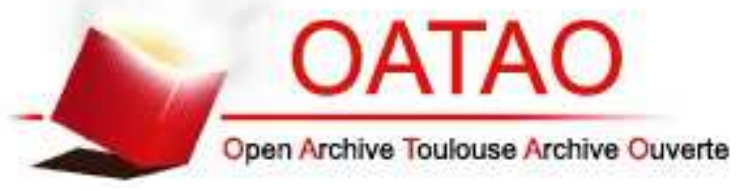

\section{Open Archive Toulouse Archive Ouverte}

OATAO is an open access repository that collects the work of Toulouse researchers and makes it freely available over the web where possible

This is an author's version published in: https://oatao.univ-toulouse.fr/26529

\section{Official URL:}

https://doi.org/10.1103/PhysRevFluids.5.073601

\section{To cite this version:}

Shi, Pengyu and Rzehak, Roland and Lucas, Dirk and Magnaudet, Jacques Hydrodynamic forces on a clean spherical bubble translating in a wall-bounded linear shear flow. (2020) Physical Review Fluids, 5 (7). 073601. ISSN 2469-990X

Any correspondence concerning this service should be sent to the repository administrator: tech-oatao@listes-diff.inp-toulouse.fr 


\title{
Hydrodynamic forces on a clean spherical bubble translating in a wall-bounded linear shear flow
}

\author{
Pengyu Shi $\odot,{ }^{1,2}$ Roland Rzehak, ${ }^{1}$ Dirk Lucas $\odot,{ }^{1}$ and Jacques Magnaudet ${ }^{3, *}$ \\ ${ }^{1}$ Helmholtz-Zentrum Dresden-Rossendorf, Institute of Fluid Dynamics, \\ Bautzner Landstrasse 400, D-01328 Dresden, Germany \\ ${ }^{2}$ Technische Universität Dresden, Faculty of Mechanical Engineering, Institute of Power Engineering, \\ D-01062 Dresden, Germany \\ ${ }^{3}$ Institut de Mécanique des Fluides de Toulouse (IMFT), Université de Toulouse, CNRS, \\ 31400 Toulouse, France
}

(Received 26 March 2020; accepted 9 June 2020; published 1 July 2020)

\begin{abstract}
The three-dimensional flow around a spherical clean bubble translating steadily in a wall-bounded linear shear flow is studied numerically. The present work is concerned with the drag and lift forces experienced by the bubble over a wide range of Reynolds number $\left(0.1 \leqslant \operatorname{Re} \leqslant 10^{3}\right.$, Re being based on the bubble diameter and relative velocity with respect to the ambient fluid), wall distance $\left(1.5 \leqslant L_{\mathrm{R}} \leqslant 8, L_{\mathrm{R}}\right.$ being the distance from the bubble center to the wall normalized by the bubble radius), and relative shear rate $(-0.5 \leqslant \mathrm{Sr} \leqslant 0.5$, Sr being the ratio between the velocity difference across the bubble and the relative velocity). Based on the above range of parameters, situations where the bubble is repelled from or attracted to the wall are both covered. The flow structure and vorticity field are analyzed to obtain qualitative insight into the interaction mechanisms at work. The drag and lift forces are computed as well. Their variations agree well with theoretical predictions available in the limit of low-but-finite Reynolds number and, when the fluid is at rest, in the potential flow limit. Numerical results and analytical expressions are combined to provide accurate semiempirical expressions for the drag and lift forces at arbitrary Reynolds number and separation distance.
\end{abstract}

DOI: 10.1103/PhysRevFluids.5.073601

\section{INTRODUCTION}

Determining the hydrodynamic force acting on bubbles, drops, and particles moving parallel to a wall in a shear flow is a problem of fundamental importance, as this configuration is involved in a variety of technical and natural systems. The presence of the wall tends to increase the drag force and more importantly causes a transverse lift force acting on the body. This transverse force may be directed towards the wall or away from it, depending on the detailed flow conditions. The lift force, although often much smaller in magnitude than the drag force, plays a crucial role in the accumulation of bubbles either close to or away from the wall [1]. This force also appears in the closure relations involved in point-particle-based Eulerian-Lagrangian approaches (e.g., Ref. [2] for bubbles) or in Eulerian-Eulerian approaches based on the interpenetrating continua concept (e.g., Ref. [3] for bubbly flows).

Asymptotic theories, laboratory experiments and direct numerical simulation (DNS) have been employed to determine near-wall hydrodynamic loads under various flow conditions. Asymptotic theories are suitable for studying low-Reynolds-number configurations (see Ref. [4] for a brief

\footnotetext{
*Corresponding author: jmagnaud@imft.fr
} 


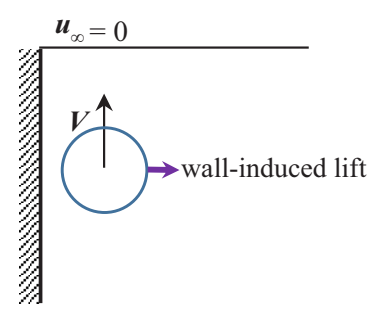

(a)

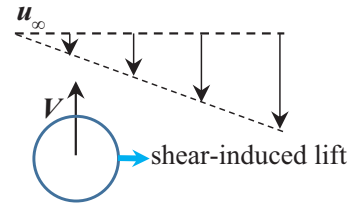

(b)

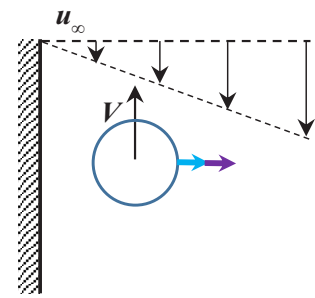

(c)

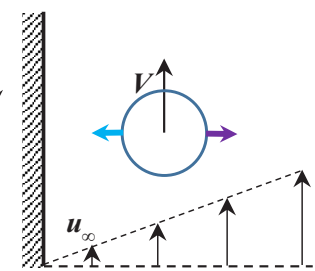

(d)

FIG. 1. Wall- and shear-induced contributions to the lift force acting on a spherical bubble in the lowto-moderate Reynolds-number regime. (a) Stagnant fluid in the presence of a wall, where only the vortical wall-induced lift exists and the bubble always leads the fluid. (b) Unbounded linear shear flow, where only the shear-induced lift exists; the force points away from the wall when the bubble lags behind the fluid, and its direction reverses if it leads the fluid. [(c), (d)] Wall-bounded linear shear flow. The bubble lags the fluid in (c), where the two effects cooperate, while it leads the fluid in (d), making the two effects combine in an antagonistic manner.

review). They may also be employed at very large Reynolds number for clean bubbles with a prescribed shape [5]. Asymptotic solutions are explicit but are valid only within a limited range of flow conditions. Experimental and DNS studies do not suffer from the same limitations and allow, in principle, arbitrary Reynolds numbers to be considered in a range of flow configurations. However, owing to mechanical limitations of the experimental devices [6,7] and numerical difficulties arising in DNS [8,9], few studies to date provide reliable data describing the variations of the lift force acting on a clean bubble moving in the vicinity of a wall as a function of the relevant parameters. In particular, no systematic investigation of shear- and wall-induced effects has been reported in the range of intermediate Reynolds numbers where inertial effects are dominant while bubbles still maintain an approximately spherical shape.

As discussed in recent reviews [10,11] and illustrated in Fig. 1, the lift force acting on a sphere translating in a wall-bounded shear flow at low-to-moderate Reynolds number arises from two primary mechanisms. The first of these is the interaction between the wall and the vorticity produced at the body surface due to its translation with respect to the ambient fluid (hereafter referred to as the wall effect). When the undisturbed fluid is stagnant, [Fig. 1(a)], this contribution reduces to a wall-normal lift force (so-called vortical wall-induced lift) pointing into the fluid [12,13]. The second mechanism is the distortion of the vorticity associated with the ambient shear by the three-dimensional body (hereafter referred to as the shear effect), which results in the generation of a nonzero vorticity component in the direction of the relative motion, concentrated within two counterrotating trailing vortices. When the body is far enough from the wall [Fig. 1(b)], this contribution reduces to the shear-induced lift force in an unbounded flow, the asymptotic expression of which was derived in Ref. [14] and [15] for a rigid sphere and a drop of arbitrary viscosity, respectively. In wall-bounded linear shear flows, both mechanisms are active. However, they do not combine linearly, as the entire flow disturbance is governed by the Oseen equation, which prevents the linear superposition of individual disturbances. Moreover, the body may either lag or lead the fluid, which, as illustrated in Figs. 1(c) and 1(d), results in a cooperative or antagonistic combination of the wall- and shear-induced lift effects. In the low-to-moderate Reynolds-number regime, bubbles and drops behave similarly to rigid spherical particles at the surface of which the no-slip condition applies, although the magnitude of the various contributions to the hydrodynamic force differs according to the viscosity ratio of the inner and outer fluids.

Things become different when the translational Reynolds number Re is large. This is because the vorticity produced at the surface of a clean bubble does not go on increasing with Re, unlike the case of a solid sphere at the surface of which the no-slip condition applies and the surface vorticity grows like $\mathrm{Re}^{1 / 2}$ when $\mathrm{Re}$ is large [16]. Because of this saturation of the surface vorticity, 


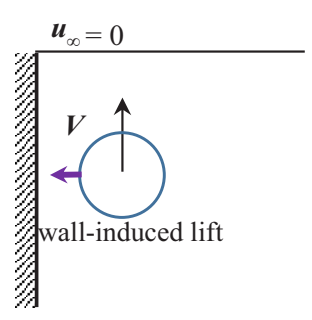

(a)

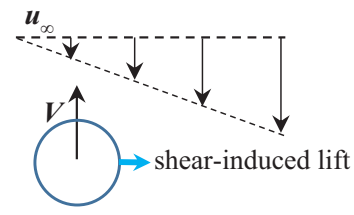

(b)

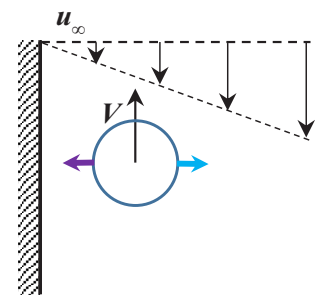

(c)

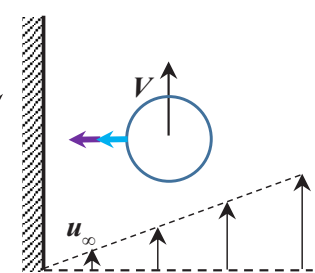

(d)

FIG. 2. Wall- and shear-induced contributions to the lift force acting on a spherical bubble in the moderateto-large Reynolds-number regime. (a) Stagnant fluid in the presence of a wall, where only the irrotational (Bernoulli) transverse mechanism exists. (b) Same as in Fig. 1(b). [(c), (d)] Wall-bounded linear shear flow. The bubble lags the fluid in (c), where the two effects act in an antagonistic manner, while it leads the fluid in (d), where they cooperate.

the magnitude of the vortical wall-induced lift force at a given distance from the wall decreases as the Reynolds number increases in the case of a clean spherical bubble. Then a second, inviscid mechanism takes over. Indeed, according to potential flow theory, a sphere translating parallel to a symmetry plane is attracted toward this plane because the fluid velocity reaches a maximum in the gap, thus inducing a pressure gradient directed away from the symmetry plane, owing to Bernoulli's theorem [17-20]. Hence, there is a critical gap-dependent Reynolds number beyond which the transverse force acting on the bubble switches from repulsive (corresponding to the above vortical wall-induced lift mechanism) to attractive (corresponding to the above irrotational mechanism) [6]. No such reversal of the transverse force takes place in the case of a solid sphere, as the vortical wall-induced mechanism remains dominant however large the Reynolds number. Compared to the low-to-moderate Re range, in the situation where the irrotational wall-induced lift mechanism is dominant [Fig. 2(a)], the wall- and-shear-induced lift forces acting on a clean bubble combine differently. As illustrated in Fig. 2(c), the two effects are now antagonistic (resp. cooperative) if the bubble lags (resp. leads) the fluid.

Owing to its fore-aft symmetry, a sphere may experience a lift force only in the presence of finite inertial effects [21]. In the low-but-finite Reynolds-number regime, when the distance $r$ to the sphere center increases, the magnitude of the translation-induced inertia term becomes comparable to the viscous term at a distance $r \sim O\left(\widetilde{L}_{u}\right)$, where $\widetilde{L}_{u}=v /\left|U_{\text {rel }}\right|$ is the Oseen length, $v$ and $U_{\text {rel }}$ denoting the fluid kinematic viscosity and relative velocity between the body and fluid, respectively. Similarly, the shear-induced inertia term becomes comparable to the viscous term at a distance $r \sim O\left(\widetilde{L}_{\omega}\right)$, where $\widetilde{L}_{\omega}=(\nu / \omega)^{1 / 2}$ is the Saffman length, $\omega=\|\omega\|$ denoting the norm of the vorticity $\omega$ in the undisturbed flow. The parameter $\varepsilon=\widetilde{L}_{u} / \widetilde{L}_{\omega}$ determines whether the dominant inertial effect arises from shear $(\varepsilon>1)$ or slip $(\varepsilon<1)$. When the flow is bounded by a flat wall, the distance $\widetilde{L}$ separating the sphere from the wall may be compared to the two inertial length scales through the ratios $L_{u}=\widetilde{L} / \widetilde{L}_{u}$ and $L_{\omega}=\widetilde{L} / \widetilde{L}_{\omega}$. Provided that $\min \left(L_{u}, L_{\omega}\right)<1$ (strictly speaking $\ll 1$ ), the wall is located in the inner region of the disturbance, while it stands in the outer region if $\max \left(L_{u}, L_{\omega}\right)>1$ (strictly speaking $\gg 1$ ). These two situations and the various length scales involved are illustrated in Fig. 3.

The wall-induced lift force acting on drops and bubbles in the low-Reynolds-number regime was considered in Ref. [4], where its asymptotic expression was obtained for situations where the wall lies in the inner region of the disturbance. The case where the wall lies in the outer region was worked out in Ref. [13] for clean bubbles, adapting the singular perturbation analysis developed in Ref. [12] for a rigid sphere. In both cases, the wall-induced lift force was predicted to decrease with increasing the inertial wall distance $L_{u}$. The wall lies usually in the outer region when the translational Reynolds number (based on the particle diameter and relative velocity with respect to the ambient fluid) is larger than unity. Experiments performed in still fluid [6] revealed that the 


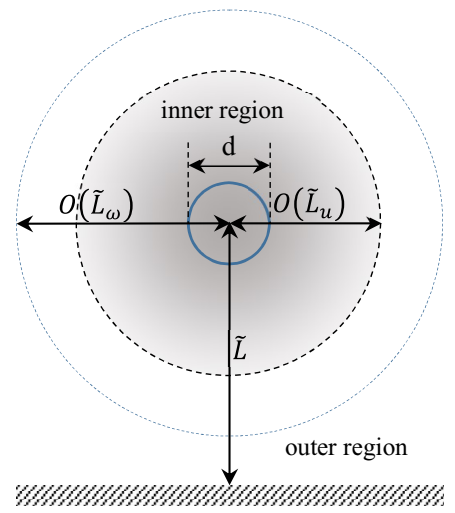

(a)

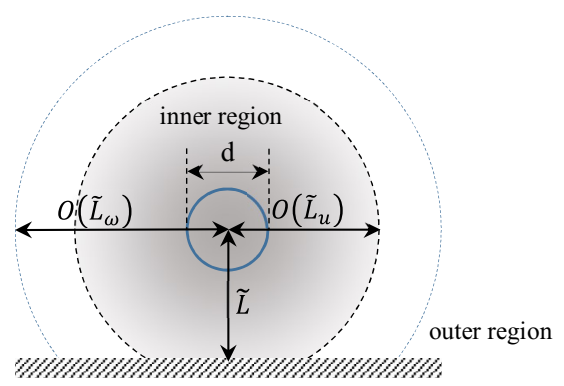

(b)

FIG. 3. Sketch of the various length scales and regions of the low-Re flow disturbance around a sphere with diameter $\mathrm{d}$ standing a distance $\widetilde{L}$ from the wall in a situation such that $\widetilde{L}_{u}<\widetilde{L}_{\omega}$, i.e., $\varepsilon<1$. The inner and outer regions correspond to distances $r$ from the sphere center such that $r<\min \left(\widetilde{L}_{u}, \widetilde{L}_{\omega}\right)$ and $r>\max \left(\widetilde{L}_{u}, \widetilde{L}_{\omega}\right)$, respectively. The wall lies in the outer region in (a), where $\widetilde{L}>\max \left(\widetilde{L}_{u}, \widetilde{L}_{\omega}\right)$, and in the inner region in (b), where $\widetilde{L}<\min \left(\widetilde{L}_{u}, \widetilde{L}_{\omega}\right)$.

vortical mechanism responsible for the wall-induced lift force dominates the interaction process for $\operatorname{Re} \lesssim 30$, while the irrotational mechanism becomes dominant at higher Re, making bubbles tend to cluster at the wall.

The analytical expression for the low-Reynolds-number shear-induced lift force acting on a rigid sphere moving in an unbounded flow domain was derived by Saffman [14,22] in the strong shear limit $(\varepsilon \gg 1)$, using matched asymptotic expansions. Extension of Saffman's expression to finite $\varepsilon$, i.e., to conditions under which advective effects due to the sphere translation cannot be neglected with respect to those due to the shear, was later conducted independently in Refs. [23] and [24] using a similar approach. The generalization of the above results to spherical drops of arbitrary viscosity was achieved in Ref. [15]. This analysis indicates that the shear-induced lift force acting on a clean bubble (i.e., a spherical drop with a vanishing inner viscosity) differs from that on a rigid sphere by only a numerical factor of $(2 / 3)^{2}$. For moderate Reynolds numbers, the vorticity generated at the bubble surface by the shear-free boundary condition and the vorticity present in the ambient shear flow both contribute to the lift force [8]. The latter becomes dominant for $\operatorname{Re} \gtrsim 10^{2}$ and the resulting lift force then approaches the inviscid prediction obtained by Auton [25].

Shear- and wall-induced lift effects combine in wall-bounded shear flows. In situations where the Reynolds number is small and the wall lies in the inner region of the disturbance, the resulting lift force acting on deforming drops of arbitrary viscosity was derived in Ref. [4] through a regular perturbation analysis. In this case, both inertia and deformation contribute to produce a nonzero lift force. The wall may lie in the outer region of the disturbance while the Reynolds number is still small, in which case the lift force may still be evaluated analytically using matched asymptotic expansions. This problem was first considered in Ref. [26] in the strong shear limit for a rigid sphere, and revisited independently in Refs. [27] and [28] where the solution was extended to finite $\varepsilon$. Both studies concluded that the presence of the wall consistently reduces the shear-induced lift force, which agrees with the behavior predicted in Ref. [4] in the case the wall lies in the inner region. In Refs. [27] and [28] the solution for the lift force was obtained in the form of a threedimensional integral in Fourier space. In Ref. [7] it was assumed that, similar to the solutions derived in Ref. [4], the lift force may still be expressed as a superposition of the wall- and the (unbounded fluid) shear-induced lift forces in physical space when the wall stands in the outer region, provided some modification due to the shear (resp. the presence of the wall) is introduced in the wall-induced 
(resp. shear-induced) contribution. This procedure yields an explicit approximate expression for the lift force as a function of the various physical parameters.

At finite Reynolds number, the wall lies usually in the outer region. Investigations in this case rely on experimental and numerical studies. Situations with bubbles rising at low-to-moderate Reynolds number along the wall of a wall-bounded shear flow, with the bubble either lagging or leading the fluid, were investigated experimentally in Ref. [7]. This allowed the authors to explore the cooperative and antagonistic interactions between the wall- and shear-induced lift effects. Using an appropriate drag law modified by the presence of the wall and the influence of inertial effects, the resulting lift force was obtained from the measured migration velocity. A semiempirical prediction for that force comparing well with the experimental data for $\mathrm{Re}<1.5$ was proposed. We are not aware of any experimental or numerical study for clean spherical bubbles moving at moderate to high Reynolds number in the same configuration.

The development of reliable approximate expressions with a wider range of applicability for the transverse force requires the acquisition of new data and an identification of the underlying mechanisms involved in the different flow regimes. In particular the proposal of Ref. [29] to conduct simulations in a moving coordinate frame to account for the translation of the body with respect to the wall possesses further potential to make progress in this direction. We apply this idea in the present work, in which we report results of a systematic numerical study performed with clean spherical bubbles translating in a wall-bounded linear shear flow with the bubble either leading or lagging the fluid. The results provided in this paper may have an important bearing on the numerical prediction of wall-bounded bubbly flows, especially because they are of direct use to improve the socalled "point-particle" models in which wall effects are usually ignored. Such improved models for bubble Lagrangian tracking may then be used to investigate situations as diverse as turbulent wallbounded bubbly flows [30], bubbly flows in vertical pipes and channels [31-34], drag reduction or enhancement by microbubbles in turbulent boundary layers [35-40], or lateral migration of bubbles in wall-bounded shear flows [41].

The paper is organized as follows. In Sec. II we formulate the problem and state more precisely the scope of the present work together with basic definitions used throughout the paper. Section III provides a short description of the numerical procedure and preliminary tests. Available expressions for the drag and lift forces available in the literature and based on either theoretical or semiempirical grounds are summarized in Sec. IV. Physical features and hydrodynamic mechanisms revealed by the flow field are discussed in Sec. V, while results for the hydrodynamic forces are presented and analyzed in Sec. VI. A summary of the results is provided in Sec. VII.

\section{STATEMENT OF THE PROBLEM}

Throughout the present work, we make use of a Cartesian coordinate system $(O x y z)$ with the origin located at the center of the bubble, as illustrated in Fig. 4. We assume that the bubble moves with a velocity $\boldsymbol{V}=V \boldsymbol{e}_{z}$ parallel to a single planar wall. The wall is located at $x=-\widetilde{L}$ and $\boldsymbol{e}_{x}$ denotes the wall-normal unit vector pointing into the fluid. In the reference frame translating with the bubble, the undisturbed flow is a one-dimensional linear shear flow with a velocity profile $\boldsymbol{u}_{\infty}=$ $[\gamma(\widetilde{L}+x)-V] \boldsymbol{e}_{z}$ and a nonzero spanwise vorticity $\boldsymbol{\omega}_{\infty}=-\gamma \boldsymbol{e}_{y}$. The relative (or slip) velocity of the fluid with respect to the bubble is then $\boldsymbol{U}_{\text {rel }}=(\gamma \widetilde{L}-V) \boldsymbol{e}_{z}$. The fluid velocity and pressure fields in the presence of the bubble are denoted by $\boldsymbol{u}$ and $p$, respectively, and $\boldsymbol{\omega}=\boldsymbol{\nabla} \times \boldsymbol{u}$ denotes the vorticity.

The suspending fluid is assumed to be Newtonian and the flow is considered incompressible. The continuity and Navier-Stokes equations thus take the form

$$
\begin{aligned}
\nabla \cdot \boldsymbol{u} & =0 \\
\frac{\partial \boldsymbol{u}}{\partial t}+\boldsymbol{u} \cdot \nabla \boldsymbol{u} & =-\frac{1}{\rho} \nabla p+\nabla \cdot \boldsymbol{\tau}
\end{aligned}
$$




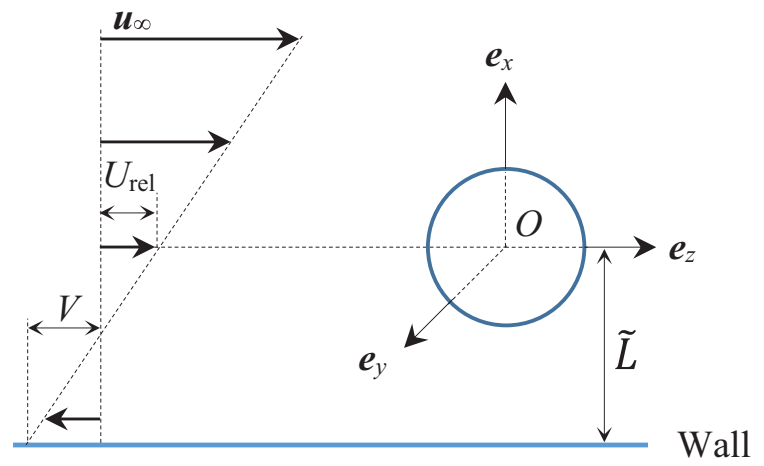

FIG. 4. Schematic of a bubble moving in a wall-bounded linear shear flow.

where $\boldsymbol{\tau}=v\left[\nabla \boldsymbol{u}+{ }^{T} \nabla \boldsymbol{u}\right]$ is the viscous part of the stress tensor $\boldsymbol{\Sigma}=-p \boldsymbol{I}+\rho \boldsymbol{\tau}$ and $\rho$ is the fluid density, $\boldsymbol{I}$ denoting the Kronecker delta. In the reference frame attached to the bubble center, boundary conditions at the wall and in the far field are

$$
\boldsymbol{u}= \begin{cases}-V \boldsymbol{e}_{z} & \text { for } x=-\widetilde{L} \\ \boldsymbol{u}_{\infty}=[\gamma(\widetilde{L}+x)-V] \boldsymbol{e}_{z} & \text { for } \quad r \rightarrow \infty\end{cases}
$$

where $r=\left(x^{2}+y^{2}+z^{2}\right)^{1 / 2}$ denotes the distance to the bubble center. On the bubble surface, the normal velocity must vanish, owing to the nonpenetration condition. Moreover, the dynamic viscosity of the gas within the bubble is assumed to be much smaller than that of the suspending liquid and the bubble surface is considered to be free of any surfactant, so that the outer fluid obeys a shear-free condition at the interface. Last, we assume surface tension to be strong enough for the capillary force to be able to maintain the bubble spherical whatever the local strength of the hydrodynamic stresses. Under such conditions, the boundary conditions at the bubble surface are

$$
\left.\begin{array}{l}
\boldsymbol{u} \cdot \boldsymbol{n}=0 \\
\boldsymbol{n} \times(\boldsymbol{\tau} \cdot \boldsymbol{n})=0
\end{array}\right\} \quad \text { for } \quad r=\frac{d}{2},
$$

where $\boldsymbol{n}$ denotes the outward unit normal to the bubble surface and $d$ is the bubble diameter. With the above boundary conditions, the steady flow field past the bubble depends on three characteristic parameters, namely the Reynolds number Re, the dimensionless shear rate $\mathrm{Sr}$, and the wall distance $L_{\mathrm{R}}$. These are defined as

$$
\operatorname{Re}=\frac{\left|U_{\text {rel }}\right| d}{v}, \quad \operatorname{Sr}=\frac{\gamma d}{U_{\text {rel }}}, \quad L_{\mathrm{R}}=\frac{2 \widetilde{L}}{d},
$$

with $U_{\text {rel }}=\boldsymbol{U}_{\text {rel }} \cdot \boldsymbol{e}_{z}$. A positive (resp. negative) Sr indicates that the bubble lags (resp. leads) the fluid.

As stated in the introduction, we are particularly interested in obtaining the hydrodynamic force acting on the bubble. This force may be split into its drag component $F_{\mathrm{D}}$ parallel to $\boldsymbol{U}_{\text {rel }}$, and its lift or transverse component, $F_{\mathrm{L}}$, parallel to $\boldsymbol{e}_{x}$. We thus define

$$
F_{\mathrm{D}}=\frac{\boldsymbol{U}_{\text {rel }}}{\left\|\boldsymbol{U}_{\text {rel }}\right\|} \cdot \int_{\Gamma} \boldsymbol{\Sigma} \cdot \boldsymbol{n} d \Gamma, \quad F_{\mathrm{L}}=\boldsymbol{e}_{x} \cdot \int_{\Gamma} \boldsymbol{\Sigma} \cdot \boldsymbol{n} d \Gamma,
$$

where $\Gamma$ is the bubble surface. Results concerning these forces will be expressed in terms of the dimensionless lift and drag coefficients, $C_{\mathrm{L}}$ and $C_{\mathrm{D}}$, obtained by dividing the corresponding component of the force by $\pi d^{2} \rho U_{\text {rel }}^{2} / 8$. Note that a negative (resp. positive) value of the lift coefficient corresponds to an attractive (resp. repulsive) force with respect to the wall. We introduce 
the notations $C_{\mathrm{D}}^{\mathrm{W}}\left(\right.$ resp. $C_{\mathrm{L}}^{\mathrm{W}}$ ) and $C_{\mathrm{D}}^{\mathrm{U}}\left(\right.$ resp. $\left.C_{\mathrm{L}}^{\mathrm{U}}\right)$ to denote the drag (resp. lift) coefficients in wall-bounded and unbounded flows, respectively. Situations where the wall lies in the inner or outer region of the disturbance will be distinguished by the superscripts $\mathrm{W}$-in and $\mathrm{W}$-out, respectively. To appreciate the modification of the drag force due to the wall and shear effects, results for the drag coefficient are usually given in the form of the relative change $\Delta C_{\mathrm{D}}=\left(C_{\mathrm{D}}-C_{\mathrm{D} 0}^{\mathrm{U}}\right) / C_{\mathrm{D} 0}^{\mathrm{U}}$, where $C_{\mathrm{D} 0}^{\mathrm{U}}$ denotes the drag coefficient of a bubble moving in an unbounded uniform stream. Drag (resp. lift) contributions corresponding to the wall-induced effect are denoted with the subscript $\mathrm{D} u$ (resp. Lu), while those corresponding to the shear-induced effect are denoted with the subscript D $\omega$ (resp. L $\omega$ ).

In most of the present work, $\mathrm{Re}, \mathrm{Sr}$, and $L_{\mathrm{R}}$ are varied in the range $\left[0.1,10^{3}\right],[-0.5,0.5],[1.5,8]$, respectively. Specifically, $\mathrm{Sr}= \pm 0.4$ corresponds for instance to a $1 \mathrm{~mm}$ diameter bubble rising or settling at $0.25 \mathrm{~m} \mathrm{~s}^{-1}$ in a downward or upward shear flow with $\gamma=100 \mathrm{~s}^{-1}$, an order of magnitude typical of turbulent boundary layers. Of course, in most practical situations, rising bubbles do not stay spherical at high Reynolds number, even in water, which has a strong surface tension. Rather, they turn into oblate spheroids, characterized by an aspect ratio, $\chi$, the length ratio of the major-to-minor axis. The dynamics of moderately oblate bubbles is essentially similar to that of spherical bubbles up to $\chi \approx 1.65$, beyond which a separated wake may exist within a certain range of Reynolds number [42]. However, an oblate bubble opposes a greater resistance to the surrounding flow than a spherical bubble with the same volume $\mathcal{V}$. For instance, in the high-Re limit, the drag and shear-induced lift coefficients are approximately $25 \%$ higher for a bubble with an aspect ratio $\chi=1.2$ than for a spherical bubble [43,44]. In pure water under standard conditions, bubbles with $\chi=1.2$ have an equivalent diameter $\mathcal{D}=1.15 \mathrm{~mm}$ (with $\mathcal{D}=(6 \mathcal{V} / \pi)^{1 / 3}$ ), and a rise velocity $V_{T} \approx 0.305 \mathrm{~ms}^{-1}$ [45]. Therefore their rise Reynolds number $\operatorname{Re}_{\mathrm{T}}=V_{T} \mathcal{D} / v$ is approximately 350 . If one regards the above $25 \%$ difference as the upper limit below which predictions for the hydrodynamic forces are reasonably accurate, results obtained with spherical bubbles may be considered valid up to a Reynolds number $\operatorname{Re} \approx 350$, with Re based on the slip velocity between the bubble and the local flow. Since we consider only shear rates such that $|\operatorname{Sr}| \leqslant 1$ here, shear-induced deformations are smaller than those due to slip, and the above conclusion extends to bubbles rising in a linear shear flow.

Although effects of oblateness cannot be ignored for bubbles rising at larger Reynolds numbers in water, there is a fundamental interest in considering fictitious conditions under which surface tension is strong enough to prevent bubbles from deforming irrespective of the Reynolds number. This is because, in contrast with the case of rigid spheres, asymptotic results are available in the limit $\operatorname{Re} \rightarrow \infty$ for spherical bubbles at the surface of which the fluid obeys a shear-free condition, making it possible to study how the corresponding asymptotic regime is reached for large-but-finite Re. This is for instance the case with the viscous drag [46,47], the shear-induced lift force [8,25], or the irrotational transverse force on a bubble rising parallel to a wall $[17,18,48]$. This is the reason why Reynolds numbers are considered up to $10^{3}$ in the present study.

Finally, although gravity does not appear in (1) or (3), it is a key player in the real system under consideration, since it usually provides the driving force that sets the relative motion between the bubble and the surrounding fluid. Equating the drag and buoyancy forces acting on a bubble rising in a fluid at rest (neglecting the gas density) yields $\operatorname{Re}_{\mathrm{T}}^{2} C_{\mathrm{D} 0}^{\mathrm{U}}\left(\mathrm{Re}_{\mathrm{T}}\right)=\frac{4}{3} g \mathcal{D}^{3} / \nu^{2}, g$ denoting gravity. The dimensionless number $\operatorname{Ar}=\left(g \mathcal{D}^{3}\right)^{1 / 2} / \nu$, frequently referred to as the Archimedes number, compares buoyancy and viscous forces. Its value, which depends only on the bubble size, fluid viscosity, and gravity conditions, determines the rise Reynolds number, $\mathrm{Re}_{\mathrm{T}}$. Gravity has also a side effect, since it tends to deform bubbles by inducing a hydrostatic pole-to-pole pressure difference of $O(\rho g \mathcal{D})$ along their surface. This deformation remains weak as far as this pressure difference is small compared to the capillary pressure jump across the bubble-fluid interface, of $O(4 \sigma / \mathcal{D})$. Introducing the Bond number Bo $=\rho g \mathcal{D}^{2} / \sigma$ ( $\sigma$ denoting surface tension), this condition may be expressed in the form $\mathrm{Bo} \ll 4$. For the $1.15 \mathrm{~mm}$ bubble considered above, one has $\mathrm{Bo} \approx 0.18$, so that the gravity-induced deformation is negligible. So, the $20 \%$ deformation corresponding to the 


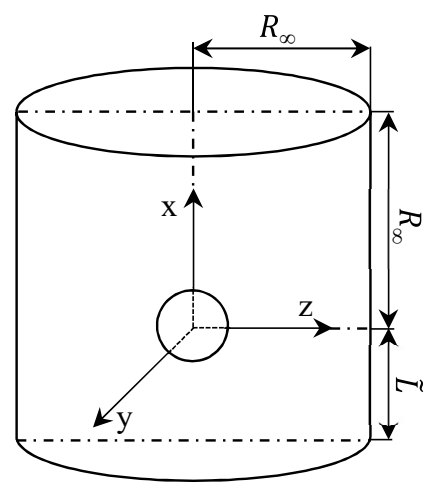

(a)

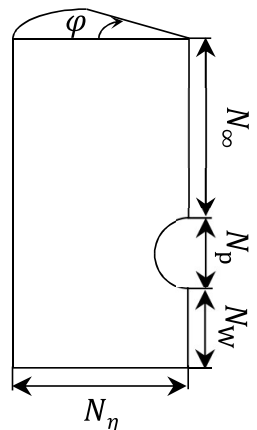

(b)

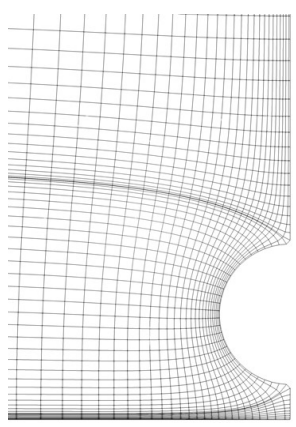

(c)

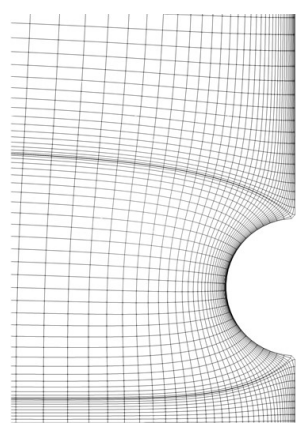

(d)

FIG. 5. Illustration of the grid system. (a) Shape and size of the computational domain. (b) Definition of the number of nodes in the computational domain. [(c), (d)] Partial view of the grid near the bubble with $L_{\mathrm{R}}=1.5$ and $2 \delta / d=0.01$ in (c), and $L_{\mathrm{R}}=2.0$ and $2 \delta / d=0.002$ in (d).

aspect ratio $\chi=1.2$ essentially results from dynamical effects, the Weber number $\mathrm{We}=\rho V_{T}^{2} \mathcal{D} / \sigma$ being approximately 1.5 in this case.

\section{NUMERICAL APPROACH}

The computational results discussed below were obtained with the JADIM code developed at IMFT. This code solves the three-dimensional unsteady Navier-Stokes equations with a finitevolume discretization and, in the version used here, makes use of a boundary-fitted staggered grid. Spatial derivatives are approximated using second-order centered schemes. The velocity field is advanced in time with a third-order Runge-Kutta algorithm for the advective term, together with a Crank-Nicolson semi-implicit algorithm for viscous terms. Incompressibility is satisfied at the end of each time step through a projection technique which yields a Poisson equation for the pressure correction. Details about this code may be found in previous publications, especially Ref. [49] for most algorithmic aspects, and Ref. [50] (resp. Ref. [48]) for specific aspects concerned with curvilinear grids in unbounded (resp. wall-bounded) flows.

The grid system used in this study is similar to the one employed in Ref. [48], based on which the flow about two spherical bubbles rising side-by-side in a viscous liquid was simulated. A twodimensional grid is first built on the streamlines $\eta=$ const and the equipotential lines $\xi=$ const of the potential flow around two circular cylinders moving in line along the $z$ axis. Then this grid is rotated about the $x$ axis with an angle $\varphi=\pi$ or $2 \pi$. The first choice, which we used in certain runs, exploits the planar symmetry of the problem geometry with respect to the plane $y=0$, reducing the computational domain to a half-space by imposing a symmetry condition in this plane. Most computations, however, were run in the full domain $0 \leqslant \varphi \leqslant 2 \pi$. There is a priori no guarantee that the flow keeps its planar symmetry at high Reynolds number, owing to the combined presence of wall and shear, but none of these computations revealed such a symmetry breaking. The resulting computational domain with $\varphi=2 \pi$, shown in Fig. 5(a), has an inner boundary coinciding with the bubble surface and a circular outer boundary of radius $R_{\infty}$. Its height between the bubble center and the wall (resp. the outer boundary) is $\widetilde{L}$ (resp. $\left.R_{\infty}\right)$. The $(\xi, \eta, \varphi)$ grid is made of $\left(N_{\mathrm{W}}+N_{\mathrm{p}}+\right.$ $\left.N_{\infty}\right) \times N_{\eta} \times N_{\varphi}$ nodes as defined in Fig. 5(b). Here $N_{\mathrm{W}}$ is the number of nodes between the wall $x=-\widetilde{L}$ and the bubble surface, $N_{\mathrm{p}}$ is the number of nodes along half of the bubble circumference (from $x=-d / 2$ to $x=d / 2$ ), $N_{\infty}$ is the number of nodes between the bubble surface and the outer boundary in the $x$ direction, $N_{\eta}$ and $N_{\varphi}$ are the number of nodes along the radial $(\eta)$ and azimuthal $(\varphi)$ directions, respectively. The bubble surface is thus discretized with $N_{\mathrm{p}} \times N_{\varphi}$ nodes. 
In Ref. [48] it was found that the length $R_{\infty}$ and the distance $\delta$ from the bubble surface to the first node above it near the singular points $x= \pm d / 2$ of the $x$ axis are critical for an accurate calculation of the transverse force. To reduce the effect of the outer boundary, we select $R_{\infty}$ such that $2 R_{\infty} / d=$ 100 for $\operatorname{Re}<1,2 R_{\infty} / d=80$ for $\operatorname{Re}<100$, and $2 R_{\infty} / d=40$ for $\operatorname{Re} \geqslant 100$. To improve accuracy in the moderate and high-Reynolds-number regimes, the dimensionless distance $2 \delta / d$ is set to 0.01 for $\operatorname{Re}<10$ and to 0.002 for $\operatorname{Re} \geqslant 10$. A constant spacing of nodes is chosen in the $\varphi$ direction with $N_{\varphi}=128$ (resp. 64) in the case of a full (resp. half) domain. In the $\xi$ and $\eta$ directions, a geometrical progression ensuring that the width ratio of two successive cells is less than 1.15 is applied, with $N_{\infty}=58$ and $N_{\eta}=54$. Some tests were carried out at large Reynolds number with a finer resolution in the radial direction, $N_{\eta}=80$ and 108. Differences on the drag and lift coefficients were found to be less than $0.4 \%$ in all cases, from which we concluded that $N_{\eta}=54$ is sufficient throughout the range of flow conditions considered here. In Ref. [48] it was shown that numerical results are weakly sensitive to the number of nodes on the bubble surface, $N_{\mathrm{p}}$, and between the bubble and the wall, $N_{\mathrm{W}}$. Regarding the former, the configuration considered here does not differ much from that in Ref. [48]. Hence we followed this reference and set $N_{\mathrm{p}}=30$. The situation is different regarding $N_{\mathrm{W}}$, since the plane $x=-\tilde{L}$ corresponds to a rigid wall with a no-slip condition here, whereas it was only a symmetry plane in Ref. [48]. For this reason, more grid points are required near the wall when the Reynolds number becomes large to properly capture the corresponding boundary layer. For $\operatorname{Re} \leqslant 10$, we found it convenient to employ $N_{\mathrm{W}}=10$ for $L_{\mathrm{R}} \leqslant 2, N_{\mathrm{W}}=15$ for $2 \leqslant L_{\mathrm{R}} \leqslant 4$, and $N_{\mathrm{W}}=20$ for $L_{\mathrm{R}}>4$. At larger Reynolds numbers, the results to be discussed later show that effects of the boundary layer are "felt" by the bubble only for short separation distances, typically $L_{\mathrm{R}} \leqslant 2$. We increased $N_{W}$ up to 40 (resp. 60) for $L_{\mathrm{R}}=1.5$ (resp. 2) and found that results are grid-independent for $N_{W} \geqslant 30$ (resp. 40) up to $\mathrm{Re}=10^{3}$. Therefore we adopted the latter values, which allow to cluster approximately 10 grid points within the wall boundary layer for $\operatorname{Re}=10^{3}$, guaranteeing a proper capture of the corresponding effects. Two examples of the near-bubble cell distribution at low-to-moderate Reynolds numbers are provided in Fig. 5. The adequacy of the resulting resolution is established in the Appendix, where we discuss results for the drag and lift forces in the wall-bounded configuration with the fluid at rest at infinity, as well as predictions for the lift force in an unbounded shear flow in the high-Reynolds-number range.

The velocity is assumed to correspond to the undisturbed flow (2) on the top plate $x=R_{\infty}$ and the cylindrical surface $\left(y^{2}+z^{2}\right)^{1 / 2}=R_{\infty}$. On the bottom plate $x=-\widetilde{L}$, the no-slip condition in the moving frame, $\boldsymbol{u}=-V \boldsymbol{e}_{z}$, is applied. The $\boldsymbol{e}_{x}$-symmetry axis of the grid system introduces an artificial singularity, since a given point $\left(x=x_{0}, y=0, z=0\right)$ on this axis is associated with different values of $\varphi$. A specific condition described in Ref. [8] is employed to overcome this problem. The techniques used to evaluate the various contributions to the hydrodynamic force experienced by the bubble may be found in the same reference.

\section{AVAILABLE ANALYTICAL AND SEMIEMPIRICAL EXPRESSIONS}

Before we discuss the numerical results, an overview of expressions available in the literature to estimate wall- and shear-induced forces, based on either theoretical or semiempirical grounds, is in order. Indeed, these results help to understand the dependence of wall- and shear-induced effects with respect to $\mathrm{Re}, \mathrm{Sr}$, and $L_{\mathrm{R}}$ and provide useful indications regarding the accuracy of the numerical results.

\section{A. Unbounded linear shear flow}

At low-but-finite Reynolds number, the presence of a uniform shear tends to increase the drag, and simultaneously causes a transverse or lift force in the direction of $\boldsymbol{U}_{\text {rel }} \times \boldsymbol{\omega}$. Both forces are 
proportional to $(|\mathrm{Sr}| \mathrm{Re})^{1 / 2}$. They may be expressed in terms of the drag and lift coefficients in the form

$$
\begin{aligned}
& C_{\mathrm{D} \omega}^{\mathrm{U}}(\operatorname{Re} \ll 1)-C_{\mathrm{D} 0}^{\mathrm{U}}(\operatorname{Re} \rightarrow 0)=\frac{8}{\pi^{2}} \varepsilon J_{\mathrm{D}}(\varepsilon), \\
& C_{\mathrm{L} \omega}^{\mathrm{U}}(\operatorname{Re} \ll 1)=\frac{8}{\pi^{2}} \frac{\mathrm{Sr}}{|\mathrm{Sr}|} \varepsilon J_{\mathrm{L}}(\varepsilon),
\end{aligned}
$$

where $C_{\mathrm{D} 0}^{\mathrm{U}}(\mathrm{Re} \rightarrow 0)$ denotes the drag coefficient in the creeping flow limit: $C_{\mathrm{D} 0}^{\mathrm{U}}(\operatorname{Re} \rightarrow 0)=16 / \operatorname{Re}$ for a clean spherical bubble, and $\varepsilon=\widetilde{L}_{u} / \widetilde{L}_{\omega}=(|\mathrm{Sr}| / \mathrm{Re})^{1 / 2}$ is the ratio of the Oseen and Saffman length scales. The function $J_{\mathrm{L}}(\varepsilon)$ was derived for arbitrary values of $\varepsilon$ in Ref. [24] [Eq. (3.20)]. In the limit $\varepsilon \rightarrow \infty$, one has [51]

$$
\begin{aligned}
& J_{\mathrm{D}}(\infty)=0.485 \\
& J_{\mathrm{L}}(\infty)=2.254 .
\end{aligned}
$$

The way $J_{D}(\varepsilon)$ tends to zero as $\varepsilon$ decreases is still unknown, but this is of little importance since the relative magnitude of the shear-induced correction to the drag is small whatever $\varepsilon$ in this regime. At finite $\varepsilon, J_{\mathrm{L}}$ may be approximated as [8]

$$
J_{\mathrm{L}}(\varepsilon) \approx J_{\mathrm{L}}(\infty)\left(1+0.2 \varepsilon^{-2}\right)^{-3 / 2}
$$

In the inviscid limit, the lift force was determined analytically in Ref. [25] for a vanishingly small shear $(\mathrm{Sr} \rightarrow 0)$. The high-Reynolds-number behavior was investigated numerically in Ref. [8], where it was found that [52]

$$
\begin{array}{r}
\Delta C_{\mathrm{D} \omega}^{\mathrm{U}}(\operatorname{Re} \gg 1) \approx 0.55 \mathrm{Sr}^{2}, \\
C_{\mathrm{L} \omega}^{\mathrm{U}}(\operatorname{Re} \gg 1) \approx \frac{2}{3} \mathrm{Sr}(1-0.07|\mathrm{Sr}|) \frac{1+16 \mathrm{Re}^{-1}}{1+29 \mathrm{Re}^{-1}},
\end{array}
$$

where $\Delta C_{\mathrm{D} \omega}^{\mathrm{U}}=\left[C_{\mathrm{D} \omega}^{\mathrm{U}}(\mathrm{Re})-C_{\mathrm{D} 0}^{\mathrm{U}}(\mathrm{Re})\right] / C_{\mathrm{D} 0}^{\mathrm{U}}(\mathrm{Re})$, and $C_{\mathrm{L} \omega}^{\mathrm{U}}=\frac{2}{3} \mathrm{Sr}$ is the asymptotic value of the lift coefficient found in the limit $\mathrm{Sr} \rightarrow 0, \operatorname{Re} \rightarrow \infty$ in Ref. [25]. The fit (9a) for the shear-induced drag increase was confirmed in subsequent numerical studies [53,54].

\section{B. Low-Re wall-bounded shear flow}

The presence of a nearby wall results in a drag increase, while it may either increase or decrease the transverse force, depending on the sign of the relative (slip) velocity with respect to the direction of the shear. For $\mathrm{Re} \ll 1$, situations where the wall lies in the inner region of the disturbance, i.e., $\max \left(L_{u}, L_{\omega}\right) \ll 1$, were investigated in Ref. [4]. The analytical solutions for the drag and lift forces were found to be [55]

$$
\begin{aligned}
\Delta C_{\mathrm{D}}^{\mathrm{W}-\mathrm{in}}\left(\mathrm{Sr}, L_{\mathrm{R}}\right)= & \underbrace{\left(\frac{3}{8} L_{\mathrm{R}}^{-1}+\frac{3}{64} L_{\mathrm{R}}^{-4}\right)\left(1-\frac{3}{8} L_{\mathrm{R}}^{-1}-\frac{3}{64} L_{\mathrm{R}}^{-4}\right)^{-1}}_{\Delta C_{\mathrm{D} u}^{\mathrm{W}-i n}}-\underbrace{\frac{1}{16}\left(L_{\mathrm{R}}^{-2}+\frac{3}{8} L_{\mathrm{R}}^{-3}\right) \mathrm{Sr}}_{\Delta C_{\mathrm{D} \omega}^{\mathrm{W}-\text { in }}}, \\
C_{\mathrm{L}}^{\mathrm{W}-\mathrm{in}}\left(\mathrm{Sr}, L_{\mathrm{R}}\right)= & \underbrace{\frac{1}{2}\left(1+\frac{1}{8} L_{\mathrm{R}}^{-1}-\frac{33}{64} L_{\mathrm{R}}^{-2}\right)}_{C_{\mathrm{L} u}^{\mathrm{W}-i n}} \\
& +\underbrace{\frac{11}{24}\left(L_{\mathrm{R}}+\frac{9}{8}-\frac{1271}{3520} L_{\mathrm{R}}^{-1}\right) \mathrm{Sr}+\frac{11}{144}\left(1+\frac{3}{8} L_{\mathrm{R}}^{-1}\right) \mathrm{Sr}^{2}}_{C_{\mathrm{L} \omega}^{\mathrm{W}-\text { in }}},
\end{aligned}
$$

where $\Delta C_{\mathrm{D}}^{\mathrm{W}-\mathrm{in}}\left(\mathrm{Sr}, L_{\mathrm{R}}\right)=\left[C_{\mathrm{D}}^{\mathrm{W}-\mathrm{in}}\left(\mathrm{Sr}, L_{\mathrm{R}}\right)-C_{\mathrm{D} 0}^{\mathrm{U}}(\operatorname{Re} \rightarrow 0)\right] / C_{\mathrm{D} 0}^{\mathrm{U}}(\operatorname{Re} \rightarrow 0)$. 


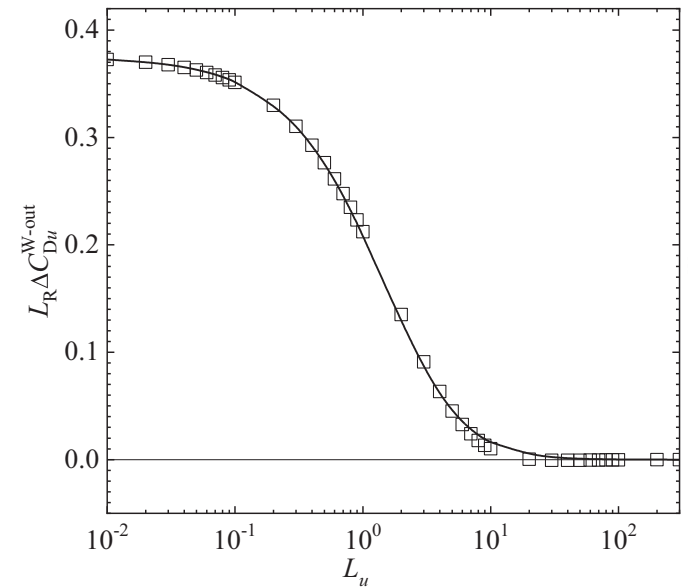

(a)

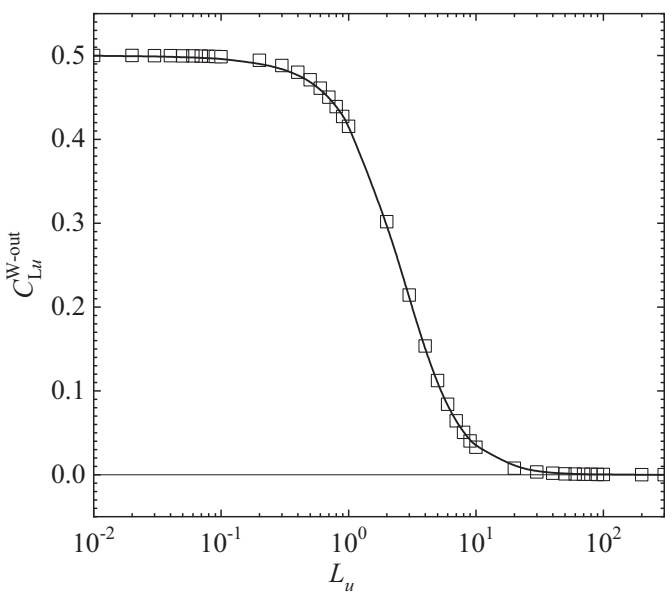

(b)

FIG. 6. Predictions for $L_{\mathrm{R}} \Delta C_{\mathrm{D} u}^{\mathrm{W}-\text { out }}$ and $C_{\mathrm{L} u}^{\mathrm{W} \text {-out }}$ based on (11) for $0.01 \leqslant L_{u} \leqslant 300$. Open symbols: numerical integration; solid line: approximate expression (12). (a) $L_{\mathrm{R}} \Delta C_{\mathrm{D} u}^{\mathrm{W}-\text { out }}$; (b) $C_{\mathrm{L} u}^{\mathrm{W} \text {-out }}$.

Equations (10) make it clear that the drag modification and the lift force result from two contributions. One, denoted as $\Delta C_{\mathrm{D} u}^{\mathrm{W}-\text { in }}$ (resp. $C_{\mathrm{L} u}^{\mathrm{W}-\mathrm{in}}$ ), is due to the wall effect and depends on $L_{\mathrm{R}}$ only. The other, denoted as $\Delta C_{\mathrm{D} \omega}^{\mathrm{W} \omega}$ (resp. $C_{\mathrm{L} \omega}^{\mathrm{W}-\mathrm{in}}$ ), is due to the presence of the shear and depends linearly and quadratically on Sr, although it is also altered by the wall proximity. The drag modification vanishes in the limit of large separations. In the same limit, the lift force becomes governed by the shear effect which grows linearly with $L_{\mathrm{R}}$. Since $L_{\omega}=\frac{1}{2} L_{\mathrm{R}}(\operatorname{Re}|\mathrm{Sr}|)^{1 / 2}$, the term proportional to $L_{\mathrm{R}}$ in the $C_{\mathrm{L} \omega}^{\mathrm{W}-\text { in }}$ contribution differs from the the lift coefficient $C_{\mathrm{L} \omega}^{\mathrm{U}}$ in $(6 \mathrm{~b})$ for the unbounded case by a factor proportional to $L_{\omega} J_{\mathrm{L}}(\varepsilon)$, which indicates that the two expressions reach a similar magnitude for some finite value of $L_{\omega}$.

When the wall lies in the outer region of the disturbance, $L_{u}=\frac{1}{2} L_{\mathrm{R}} \mathrm{Re}$ and $L_{\omega}$ are not small any more, so that the drag and lift forces depend on $L_{u}$ and $L_{\omega}$ in addition to $L_{\mathrm{R}}$. This situation was investigated in the shearless case $\left(L_{\omega} \rightarrow 0\right)$ in Ref. [12] for a rigid sphere, then adapted to a spherical bubble in Ref. [13]. The relevant solutions are found in the form of double integrals which cannot be evaluated in closed form,

$$
\begin{aligned}
\Delta C_{\mathrm{D} u}^{\mathrm{W}-\text { out }}= & \frac{1}{2 \pi L_{\mathrm{R}} L_{u}} \int_{0}^{2 \pi} \int_{0}^{\infty}\left\{\left[(\chi+\lambda) e^{-2 \lambda}+2 \chi e^{-2 \chi}-4 \chi e^{-(\chi+\lambda)}\right] \frac{i \cos \phi}{\chi-\lambda}\right. \\
& \left.+\frac{i \lambda \cos \phi-L_{u}}{\chi} e^{-2 \chi}\right\} \lambda \mathrm{d} \lambda \mathrm{d} \phi, \\
C_{\mathrm{L} u}^{\mathrm{W} \text {-out }}= & \frac{4}{\pi L_{u}^{2}} \int_{0}^{2 \pi} \int_{0}^{\infty} \frac{\chi+\lambda}{\chi-\lambda}\left(e^{-\lambda}-e^{-\chi}\right)^{2} \lambda d \lambda d \phi,
\end{aligned}
$$

where $\quad \Delta C_{\mathrm{D} u}^{\mathrm{W} \text {-out }}(\operatorname{Re} \ll 1)=\left[C_{\mathrm{D} u}^{\mathrm{W} \text { out }}\left(L_{u}, L_{\mathrm{R}}\right)-C_{\mathrm{D} 0}^{\mathrm{U}}(\operatorname{Re} \rightarrow 0)\right] / C_{\mathrm{D} 0}^{\mathrm{U}}(\operatorname{Re} \rightarrow 0), \quad$ and $\quad \chi^{2}=\lambda^{2}+$ $i \lambda L_{u} \cos \phi$ with $i^{2}=-1$.

The right-hand side of (11) may be evaluated numerically using an adaptive integration approach [56]. The corresponding values for the drag and lift coefficients, shown in Figs. 6(a) and 6(b), respectively, may then be used to derive approximate fits, which we obtained in the form

$$
\begin{aligned}
& \frac{8}{3} L_{\mathrm{R}} \Delta C_{\mathrm{D} u}^{\mathrm{W} u \text { out }}(\operatorname{Re} \ll 1)=f_{\mathrm{D}}^{\prime}\left(L_{u}\right) \approx \frac{1}{1+0.16 L_{u}\left(L_{u}+4\right)}, \\
& 2 C_{\mathrm{L} u}^{\mathrm{W}-\text { out }}(\operatorname{Re} \ll 1)=f_{\mathrm{L}}^{\prime}\left(L_{u}\right) \approx \frac{1}{1+0.13 L_{u}\left(L_{u}+0.53\right)} .
\end{aligned}
$$


The two functions $f_{\mathrm{D}}^{\prime}$ and $f_{\mathrm{L}}^{\prime}$, shown in Fig. 6, describe how the wall-induced drag modification and the transverse force relax to zero as inertial effects in the bulk become dominant. In the limit $L_{u} \rightarrow 0$, expressions (12a) and (12b) match predictions (10a) and (10b) taken in the limit $L_{\mathrm{R}} \rightarrow \infty$.

In the presence of shear, the case where the wall stands in the outer region of the disturbance was worked out in Ref. [28] for a rigid sphere. Again, the solution was obtained in the form of a volume integral in Fourier space, the value of which cannot be put in closed form but was tabulated for various values of $L_{\omega}$ and $\varepsilon=\widetilde{L}_{u} / \widetilde{L}_{\omega}$. These results were adapted to the case of a bubble in Ref. [7]. Variations of the volume integral with respect to $L_{\omega}$ and $\varepsilon$ were fitted to obtain tractable estimates of the lift force, assuming that this force may approximately be expressed as the superposition of the wall-induced effect and the unbounded shear-induced lift, provided each contribution is suitably modified to account for the effect of the shear and the presence of the wall, respectively. The resulting fit was obtained in the form

$$
C_{\mathrm{L}}^{\mathrm{W}-\text { out }}(\operatorname{Re} \ll 1) \approx f_{\mathrm{L}} C_{\mathrm{L} u}^{\mathrm{W} \text {-out }}(\operatorname{Re} \ll 1)+h_{\mathrm{L}} C_{\mathrm{L} \omega}^{\mathrm{U}}(\operatorname{Re} \ll 1),
$$

with $C_{\mathrm{L} \omega}^{\mathrm{U}}(\operatorname{Re} \ll 1)$ given by $(6 \mathrm{~b})$ and $C_{\mathrm{L} u}^{\mathrm{W} \text {-out }}(\mathrm{Re} \ll 1)$ approximated by $(12 \mathrm{~b})$. Appropriate fits for $f_{\mathrm{L}}$ and $h_{\mathrm{L}}$ were found to be

$$
f_{\mathrm{L}}\left(L_{\omega}, \varepsilon\right)=e^{-0.22 \varepsilon^{0.8} L_{\omega}^{2.5}}, \quad h_{\mathrm{L}}\left(L_{\omega}, \varepsilon\right)=1-e^{-\frac{11}{96} \pi^{2} \frac{L_{\omega}}{\mathrm{L}_{\mathrm{L}}(\varepsilon)}}
$$

with $J_{\mathrm{L}}(\varepsilon)$ as given by (8). In the double limit $L_{u} \rightarrow 0$ and $L_{\omega} \rightarrow 0$, the above expressions make the leading-order contributions to $C_{\mathrm{L}}^{\mathrm{W}-o u t}$ in (13) match the leading-order terms of the inner solution $C_{\mathrm{L}}^{\mathrm{W}-i n}$ in (10b). No approximate expression for the drag force modification seems available in the literature under similar flow conditions, although starting from (6a) and (12a), it can certainly be built using the same procedure.

\section{Approximate expression for the lift force at moderate-to-large Reynolds number}

No theoretical solution for the hydrodynamic forces may be obtained when inertial effects become dominant. Reliable extensions of the low-Reynolds-number and inviscid solutions to this intermediate regime may however be achieved provided that sufficient and accurate data are available. Experimental and numerical studies [6,9] devoted to the rise of a clean spherical bubble close to a wall revealed that, for $\operatorname{Re}=O(10)$, the lift force exhibits a faster decay with increasing $L_{u}$ compared to the low-Re solution. In Ref. [6], experimental observations performed with bubbles such that $\operatorname{Re}<30$ and theoretical considerations about the nature of the bubble-wall interaction could be summarized through the following semiempirical relation [57]

$$
C_{\mathrm{L} u}^{\mathrm{W}}[\operatorname{Re}=O(1-10)] \approx b^{2}\left(L_{\mathrm{R}} / 3\right)^{g} C_{\mathrm{L} u}^{\mathrm{W}-\text { out }}(\operatorname{Re} \ll 1),
$$

with

$$
\begin{aligned}
b & =1+2 \tanh \left[0.17 \mathrm{Re}^{0.4}-0.12 \mathrm{Re}^{0.05}\right] \\
\text { and } g & =-2.0 \tanh (0.01 \mathrm{Re}) .
\end{aligned}
$$

The factor $b$ expresses the increase of the vorticity at the bubble surface with the Reynolds number. Indeed the maximum of the surface vorticity is three times larger in the limit $\operatorname{Re} \rightarrow \infty$ than in the zero-Re limit. The exponent $g$ accounts for the increase of the decay rate of the lift force with $L_{u}$ as Re increases, from $L_{u}^{-2}$ at low-but-finite $\operatorname{Re}$ to $L_{u}^{-4}$ in the inviscid limit [6]. At higher Reynolds numbers, the irrotational mechanism illustrated in Fig. 2(a) becomes dominant. In the inviscid limit, the transverse force becomes identical to that experienced by each of the two bubbles in a pair rising perpendicular to its line of centers. The corresponding coefficient is obtained in the form of an infinite series with respect to the inverse of the separation distance, the first terms of which are $[17,18,48]$

$$
C_{\mathrm{L} u}^{\mathrm{W}}(\mathrm{Re} \rightarrow \infty) \approx-\frac{3}{8} L_{\mathrm{R}}^{-4}\left[1+\frac{1}{8} L_{\mathrm{R}}^{-3}+\frac{1}{6} L_{\mathrm{R}}^{-5}\right]+O\left(L_{\mathrm{R}}^{-10}\right)
$$


Still for a bubble pair rising in the above configuration, the transverse interaction yields an asymmetry of the vorticity distribution within the boundary layer surrounding each bubble. For this reason, a viscous correction arises in the transverse force at large-but-finite Re, yielding [5,48]

$$
C_{\mathrm{L} u}^{\mathrm{W}}(\operatorname{Re} \gg 1) \approx C_{\mathrm{L} u}^{\mathrm{W}}(\operatorname{Re} \rightarrow \infty)+15 \operatorname{Re}^{-1} L_{\mathrm{R}}^{-4} .
$$

Returning to the problem of a single bubble translating parallel to a wall, the transition from the low-to-moderate-Re regime corresponding to Fig. 1(a) to the high-Re regime illustrated in Fig. 2(a) was discussed in Ref. [6]. It was shown that the two driving mechanisms superimpose almost linearly, so that this transition is correctly captured by the empirical expression

$$
\begin{aligned}
C_{\mathrm{L} u}^{\mathrm{W}} & \approx C_{\mathrm{L} u}^{\mathrm{W}}[\operatorname{Re}=O(1-10)]+c_{T \infty} C_{\mathrm{L} u}^{\mathrm{W}}(\operatorname{Re} \rightarrow \infty), \\
\text { with } \quad c_{T \infty} & =1-e^{-0.22 \mathrm{Re}^{0.45}} .
\end{aligned}
$$

As Fig. 8 of Ref. [6] shows, predictions based on this linear fit compare well with the experimental values of the lift coefficient determined for $L_{R} \geqslant 4$ up to $R e=10^{2}$, the largest Reynolds number considered in that study.

Effects of the shear on the lift force for $\operatorname{Re}=O(1)$ were investigated experimentally in Ref. [7]. Based on the corresponding data, it was concluded that the linear superposition assumed in (13), which applies theoretically only for $\operatorname{Re} \ll 1$, still holds for $\mathrm{Re}=O(1)$, provided the bubble deformation remains small. To the best of our knowledge, no empirical expression for $C_{\mathrm{L}}^{\mathrm{W}}(\operatorname{Re})$ at higher Reynolds number in the presence of shear is available in the literature.

\section{CHARACTERISTICS OF THE FLOW FIELD AND FUNDAMENTAL MECHANISMS}

Figure 7(a) shows the computed profiles of the streamwise velocity disturbance along the line $(y=0, z=0)$ perpendicular to the wall ( $x$ axis) when the bubble translates in a stagnant fluid, for different separation distances and Reynolds numbers ranging from 0.1 to 500 . Note that $U_{\text {rel }}$ is negative if the bubble rises towards positive $z$, so that negative (resp. positive) fluid velocities normalized by $U_{\text {rel }}$ in this figure correspond to an upward (resp. downward) fluid motion with respect to the bubble. At high Reynolds number $(\mathrm{Re}=500)$ the disturbance decreases rapidly as the distance to the bubble increases. In the gap $\left[-L_{\mathrm{R}}<2 x / d \leqslant-1\right]$, this decrease results in a pressure gradient directed towards the bubble, hence a transverse force toward the wall, in line with the mechanism illustrated in Fig. 2(a). Due to the $L_{\mathrm{R}}^{-4}$ decay of the wall-induced pressure asymmetry [the footprint of which directly appears in (17)], wall effects are almost negligible for $L_{\mathrm{R}}=4$ but are well visible for $L_{\mathrm{R}}=2$. Compared to the former case, the disturbance is larger in the gap in the latter one, except within the thin boundary layer adjacent to the wall, in which it vanishes rapidly to satisfy the no-slip condition. When the Reynolds number decreases, so does the magnitude of the disturbance close to the bubble, owing to the growing influence of the boundary layer that develops at its surface (compare the profiles found for $\mathrm{Re}=500$ and 100). This boundary layer is totally distinct from the one that develops at the wall, being a direct consequence of the shear-free condition at a curved interface [58]. At $\mathrm{Re}=10$, the disturbance changes sign close to the bubble, within a layer whose thickness is of the order of the bubble radius. At small Reynolds number $(\operatorname{Re}=0.1)$ it is negative everywhere along the line $(y=0, z=0)$, i.e., directed along the direction of the bubble rise. The wall proximity significantly decreases the disturbance in the gap, as may be seen by comparing its magnitude on the left and right halves of the red and green lines at a given distance from the bubble center.

The influence of the shear is illustrated in Fig. 7(b), based on the results obtained with two opposite shear rates, $\mathrm{Sr}= \pm 0.5$, for a separation distance $L_{\mathrm{R}}=4$. At high enough Reynolds number ( $\operatorname{Re}=500$ or 100), the presence of a positive (resp. negative) shear, corresponding to a configuration where the bubble lags (resp. leads) the fluid, increases (resp. decreases) the magnitude of the disturbance in the gap, while it decreases (resp. increases) it on the opposite side. At moderate Reynolds number $(\operatorname{Re}=10)$, this behavior subsists outside the boundary layer surrounding the 


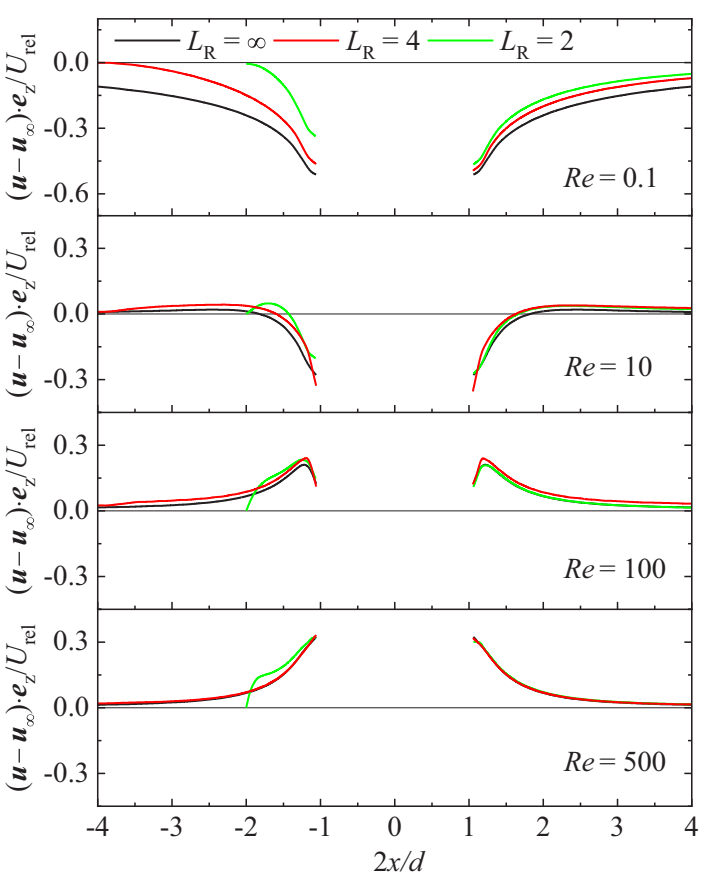

(a)

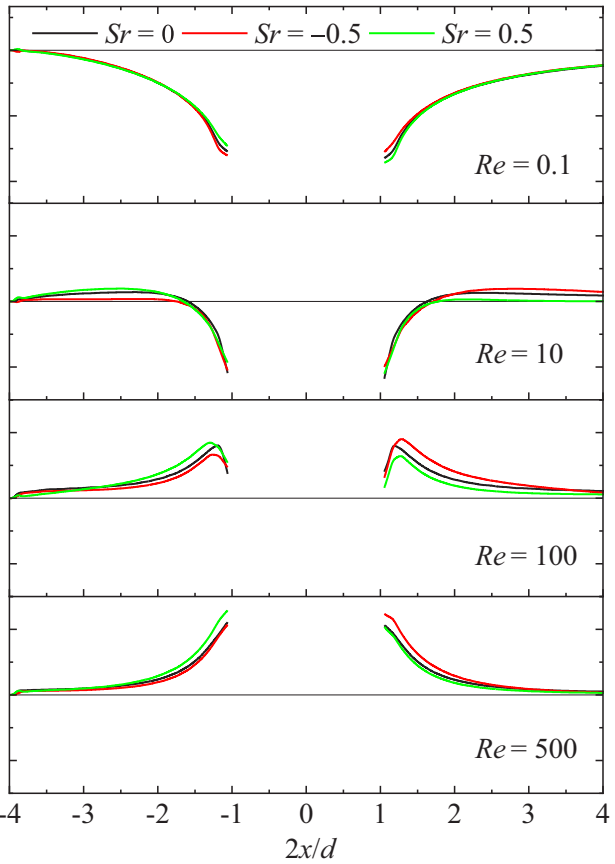

(b)

FIG. 7. Distribution of the streamwise velocity disturbance $\left(\boldsymbol{u}-\boldsymbol{u}_{\infty}\right) \cdot \boldsymbol{e}_{z} / U_{\text {rel }}$ along the line $(y=0, z=0)$. (a) Stagnant fluid ( $\mathrm{Sr}=0$ ) for different separation distances; (b) linear shear flow for $L_{\mathrm{R}}=4$. The wall stands at position $2 x / d=-2$ (resp. -4 ) for $L_{\mathrm{R}}=2$ (resp. 4).

bubble, within which only a tiny influence of Sr can be observed. In contrast, effects of the shear are detected only close to the bubble when the Reynolds number is low $(\operatorname{Re}=0.1)$. There, a positive (resp. negative) shear decreases (resp. increases) the magnitude of the disturbance in the gap, while it increases (resp. decreases) it on the opposite side.

Some aspects of these variations, and complementary features of the bubble-wall interaction, may be better understood by examining the distribution of the $y$ component of the vorticity disturbance in the symmetry plane $y=0$. This distribution is displayed in Fig. 8 for the specific separation $L_{\mathrm{R}}=2$. Vorticity is generated both at the bubble surface (due to the shear-free condition) and at the wall (due to the no-slip condition). In what follows, the contributions provided by these two distinct boundaries are referred to as surface vorticity and wall vorticity, respectively. The surface vorticity diffuses around the bubble and is advected downstream in the wake. When the fluid is at rest at infinity, the vorticity distribution in the high-Reynolds-number regime $[\mathrm{Re}=500$, Fig. 8(k)] is almost symmetric with respect to the plane $x=0$. Negative vorticity (see the sign convention in the caption of Fig. 8) is generated at the wall, owing to the weakening of the disturbance by the no-slip condition. However, in the high-Re regime, the wall shear layer is too thin to affect the distribution of the surface vorticity, the isocontours of which would be virtually unchanged if the bubble were rising in an unbounded expanse of fluid. Because of this almost symmetrical vorticity distribution, it may be inferred that the asymmetry of the disturbance velocity observed in Fig. 7(a) is mostly due to the irrotational mechanism illustrated in Fig. 2(a). When the Reynolds number decreases, the thickness of the two boundary layers increases $[\operatorname{Re}=100$, Fig. 8(h)]. Then they start to interact, making the asymmetry between the surface vorticity isocontours in the gap $(x<0)$ and those on the opposite side of the bubble $(x>0)$ more prominent $[\operatorname{Re}=10$, Fig. 8(e)]. At low Reynolds number $[\mathrm{Re}=0.1$, Fig. 8(b)], the region containing positive vorticity originating at the bubble surface and that containing negative wall vorticity interpenetrate each other, making 


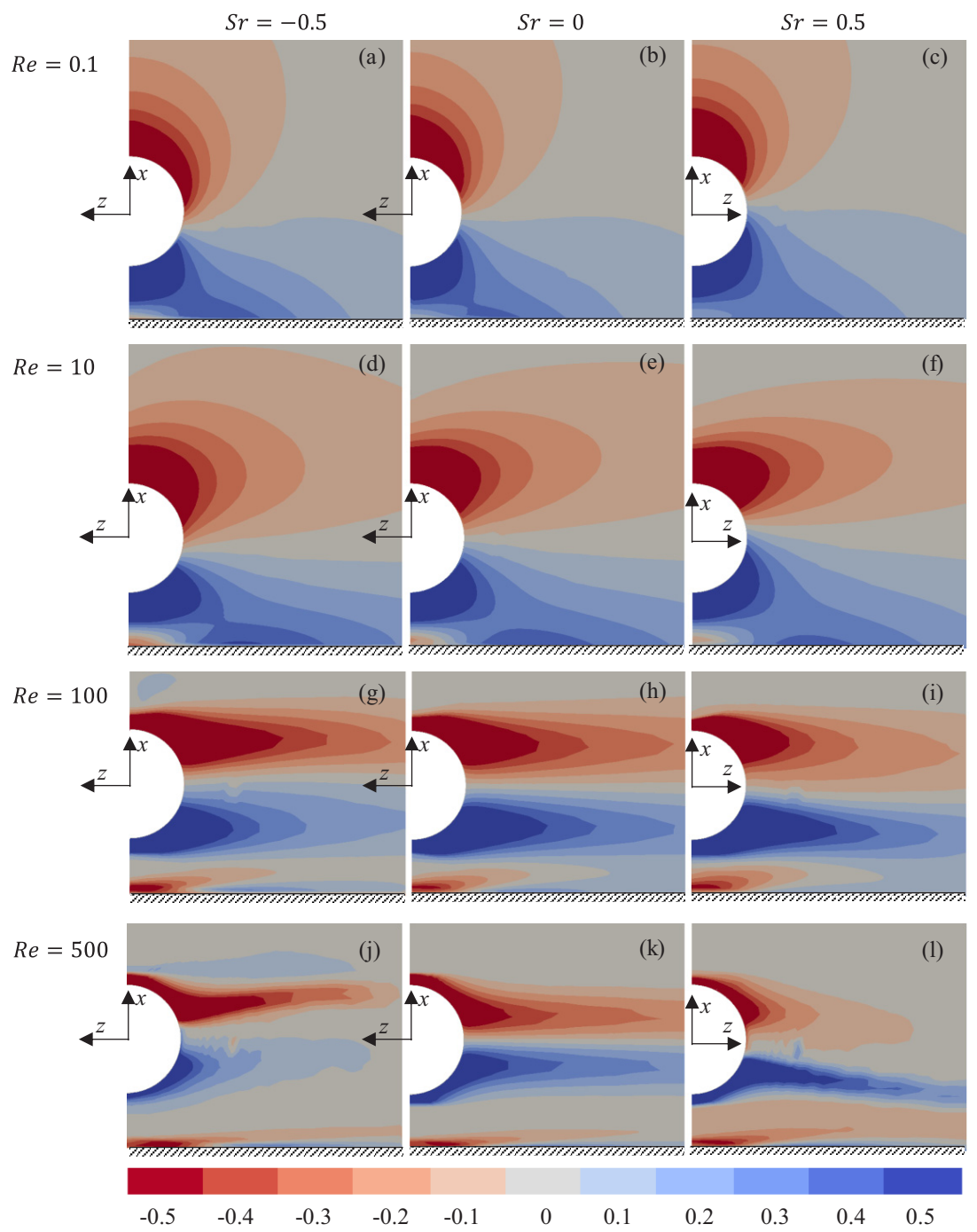

FIG. 8. Isocontours of the normalized vorticity disturbance $d /\left(2 U_{\text {rel }}\right)\left(\boldsymbol{\omega}-\boldsymbol{\omega}_{\infty}\right) \cdot \boldsymbol{e}_{y}$ in the symmetry plane $y=0$ at $L_{\mathrm{R}}=2$. Left column: $\mathrm{Sr}=-0.5\left(U_{\text {rel }}<0\right)$; central column: $\mathrm{Sr}=0\left(U_{\text {rel }}<0\right)$; right column: $\mathrm{Sr}=0.5$ $\left(U_{\text {rel }}>0\right)$. The wall stands at the bottom of each panel. The relative flow with respect to the bubble is from left to right, i.e., in the $+z$ (resp. $-z$ ) direction for $\mathrm{Sr}=0.5$ (resp. $\mathrm{Sr}=0$ and -0.5 ).

the resulting vorticity magnitude in the gap weaker than on the opposite side, especially around the midplane $z=0$. This asymmetry is responsible for the drag enhancement described by the contribution $\Delta C_{\mathrm{D} u}^{\mathrm{W}-\mathrm{in}}$ in (10a). In contrast, it is not directly responsible for the transverse force on the bubble, owing to the reversibility of the flow in the Stokes region of the disturbance. The crucial process responsible for this force component is the gradual slowing down of the fluid displaced along the wall by the bubble translation as the downstream distance increases. This slowing down corresponding to a negative $\partial_{z} u_{z}$ near the wall, continuity implies the generation of a transverse flow with a positive $\partial_{x} u_{x}$. As $u_{x}=0$ at the wall, this tiny transverse flow is directed away from the wall, yielding a pressure decrease as $x$ increases. At downstream distances larger than the Oseen length 


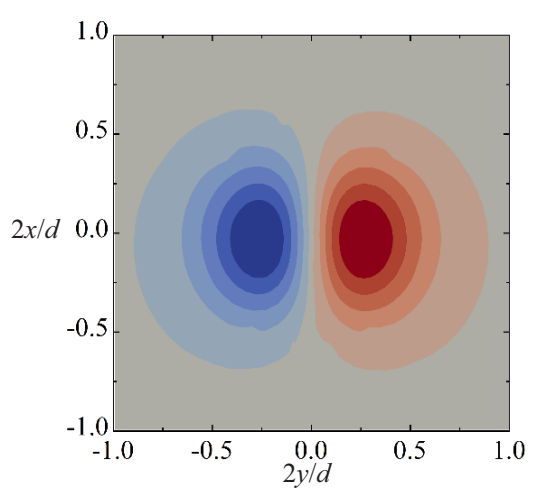

(a)

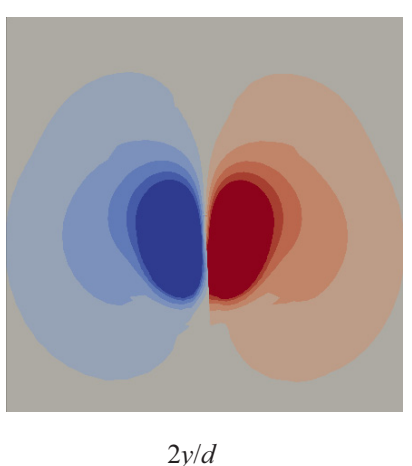

(b)

\begin{tabular}{lllllllllll}
\hline-0.25 & -0.2 & -0.15 & -0.1 & -0.05 & 0 & 0.05 & 0.1 & 0.15 & 0.2 & 0.25
\end{tabular}

FIG. 9. Isocontours of the normalized streamwise vorticity component $d /\left(2\left|U_{\text {rel }}\right|\right) \boldsymbol{\omega} \cdot \boldsymbol{e}_{z}$ in the crosssectional plane $z=d$ in an unbounded linear shear flow with $\mathrm{Sr}=0.5$. (a) $\operatorname{Re}=100$; (b) $\operatorname{Re}=500$. The main flow is going inwards, along the $z$ axis.

$L_{u}$ (here $L_{u} \approx 10 d$ ), this process is no longer reversible, yielding a repulsive net transverse force on the bubble.

The interaction between a uniformly sheared flow in an unbounded fluid and a three-dimensional bluff body is well understood in the inviscid limit (e.g., Ref. [42] and references therein). A key result of this interaction is the tilting of the upstream vorticity, $\omega_{\infty} \cdot \boldsymbol{e}_{y}=-\gamma$, by the transverse gradients of the streamwise velocity, $\partial_{y} u_{z}$, resulting from the flow around the body. This tilting mechanism yields the generation of a nonzero streamwise (or trailing) vorticity component in the wake, concentrated within the two threads of a horseshoe vortex. Figure 9 shows how the streamwise vorticity $\omega_{z}$ is distributed within a cross-sectional $(x, y)$ plane located in the near wake of the bubble. Since $\boldsymbol{\omega}_{\infty} \cdot \boldsymbol{e}_{y}<0$ and $U_{\text {rel }}>0$, the resulting $\omega_{z}$ is positive (resp. negative) for $y<0$ (resp. $y>0$ ) if $\mathrm{Sr}$ is positive; the sign of $\omega_{z}$ in each thread of the vortex reverses if $\mathrm{Sr}$ is negative. Consequently, the fluid located between the two threads is entrained downwards (resp. upwards) if $\mathrm{Sr}>0$ (resp. $\mathrm{Sr}<$ 0 ), while the fluid located outside the double-threaded wake is entrained in the opposite direction. This is the classical mechanism responsible for the inviscid lift force on a three-dimensional body, illustrated in Fig. 2(b), which yields the result (9b) for a sphere [25]. The footprint of this mechanism may be found in Figs. 8(j) and 8(1), where it is seen that the spanwise vorticity generated at the bubble surface is advected asymmetrically with respect to the plane $x=0$. Indeed, at such large Reynolds number $(\mathrm{Re}=500)$, the aforementioned downward (resp. upward) flow generated in the midplane $y=0$ by the double-threaded wake bends the isocontours of the spanwise vorticity in the wake towards the $x<0$ (resp. $x>0$ ) direction when the bubble lags (resp. leads) the fluid. Although this asymmetry arises in the wall vicinity in the present case, the wall plays no role in the underlying process.

Figure 10 reveals the spatial structure of the streamwise vorticity distribution in the presence of a nearby wall. Close to the bubble, the two halves of the horseshoe vortex are already visible in the selected diametrical plane, and the sign of $\omega_{z}$ within each of them reverses with the sign of $\mathrm{Sr}$ as expected. When $\mathrm{Sr}=0$, two thin layers with significant values of $\omega_{z}$ develop close to the lower half of the bubble surface. This is because the streamwise fluid velocity $u_{z}$ increases with the distance from the wall within the wall boundary layer, so that the lower part of the bubble is still submitted to a nonzero shear $\left(\partial_{x} u_{z} \neq 0\right)$, even though there is no shear in the upstream flow. The larger the Reynolds number, the thinner the wall boundary layer, which is why the two $\omega_{z}$ layers are much thinner and the magnitude of the streamwise vorticity within each of them is significantly 


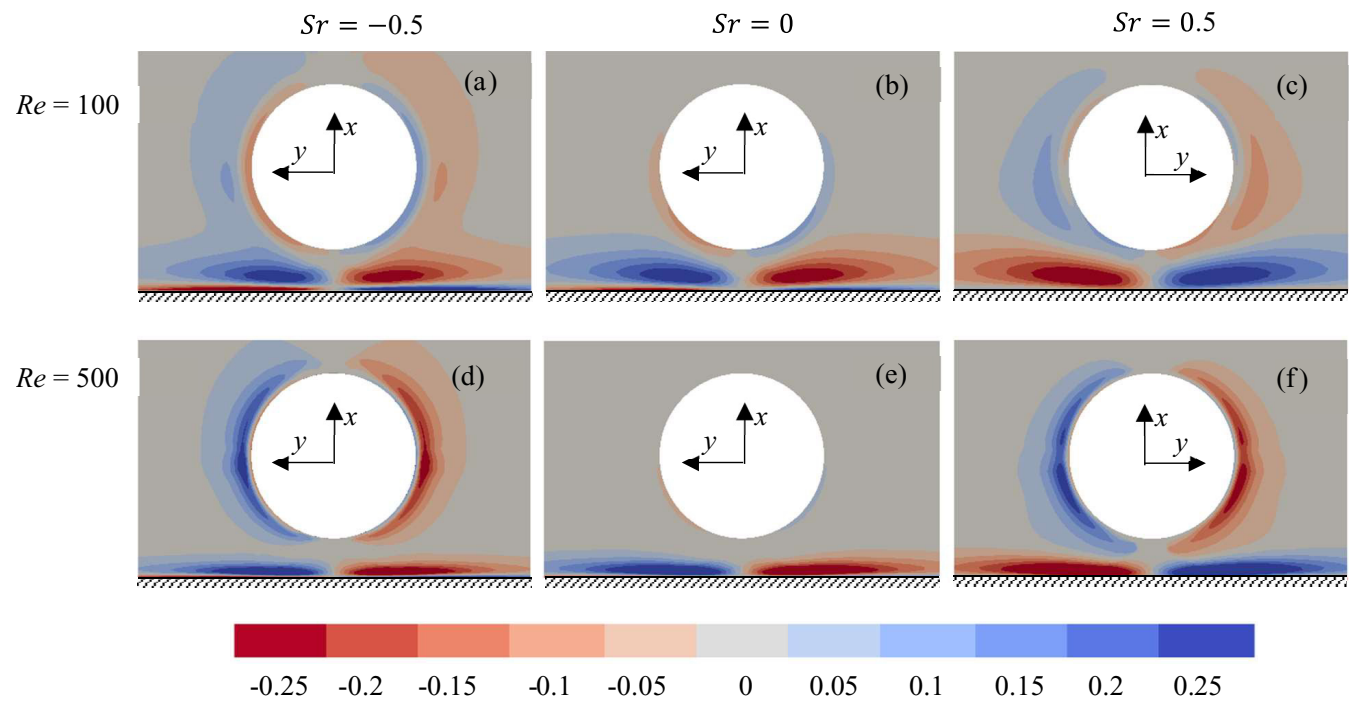

FIG. 10. Isocontours of the normalized streamwise vorticity component $d /\left(2\left|U_{\text {rel }}\right|\right) \boldsymbol{\omega} \cdot \boldsymbol{e}_{z}$ in the diametrical plane $z=0$ in a wall-bounded linear shear flow, with the bubble standing a distance $L_{\mathrm{R}}=1.5$ from the wall. The main flow is going inwards, along the $z$ (resp. $-z$ ) direction for $\mathrm{Sr}>0$ (resp. $\mathrm{Sr} \leqslant 0$ ); the direction of the $y$ axis is adjusted such that the $(x, y, z)$ coordinate system remains right-handed in all configurations.

weaker when $\mathrm{Re}=500$. This mechanism subsists when $\mathrm{Sr} \neq 0$, and then combines with the tilting mechanism associated with the outer shear. When $\mathrm{Sr}$ is positive, the two mechanisms cooperate, yielding a constant sign of $\omega_{z}$ on each side of the bubble [Figs. 10(c) and 10(f)]. In contrast, they induce opposite signs of $\omega_{z}$ when $\mathrm{Sr}$ is negative. This is visible in Fig. 10(a), where a thin near-surface layer with $\omega_{z}<0$ (resp. $>0$ ) is encapsulated within a thicker layer of positive (resp. negative) $\omega_{z}$ in the half-plane $y>0$ (resp. $y<0$ ). Clearly the inner layer results from the positive near-wall shear, while the outer layer is due to the negative upstream shear. A salient feature in Fig. 10 is the presence of two counterrotating "pancake" streamwise vortices within the wall boundary layer. These structures merely result from the spanwise displacement of near-wall fluid particles as the bubble moves parallel to the wall. Indeed, whatever the sign of Sr, fluid particles standing between the bubble and the wall are displaced sideways towards positive (resp. negative) $y$ positions in the half-plane $y>0$ (resp. $y<0$ ), implying positive (resp. negative) spanwise local velocities. Owing to the no-slip condition, $u_{y}$ vanishes at the wall, so that $\partial_{x} u_{y}$ is positive (resp. negative) near the wall in the half-plane $y>0$ (resp. $y<0)$, which yields $\omega_{z}>0$ (resp. $<0$ ). Clearly this process is independent of the outer shear, as the constant sign of $\omega_{z}$ in each half-plane irrespective of Sr confirms (note that in Figs. 9 and 10 the vorticity is normalized by $d / 2\left|U_{\text {rel }}\right|$, not $d / 2 U_{\text {rel }}$ as in Fig. 8).

The generation of the surface vorticity $\left[\omega_{y}\right.$ in the diametrical $(x, z)$ plane] is also modified by the shear. Indeed, for the shear-free condition to be satisfied at the bubble surface in the presence of an outer shear, a shear-flow type correction has to take place within the boundary layer. Compared to the unsheared case, this correction yields an additional positive vorticity component therein when the bubble lags the fluid. Hence this vorticity correction cooperates with the primary positive vorticity in the part of the bubble boundary layer corresponding to $x<0$, whereas it mitigates the primary negative vorticity on the opposite side. Consequently, the intensity of the overall vorticity round the bubble is increased for $x<0$ and decreased for $x>0$, as Fig. 8(1) indicates, and the same happens to the disturbance flow close to the bubble surface [see, e.g., the profile corresponding to $\mathrm{Re}=100$ in Fig. 7(b)]. These conclusions reverse when the bubble leads the fluid, so that the surface vorticity and streamwise velocity disturbance are lowered for $x<0$ and enhanced for 
$x>0$, in line with the distributions displayed in Figs. 8(j) and 7(b). Again, the wall plays little role in these mechanisms as far as the Reynolds number is large. More specifically, for $\operatorname{Re}=500$, Fig. 8(j) indicates that the flow region in which the vorticity originating from the bubble surface takes significant values is disconnected from the wall shear layer when $\mathrm{Sr}$ is negative, while there may be some weak connection between the two regions when $\mathrm{Sr}$ is positive [Fig. 8(1)], owing to the bending of the wake toward the wall.

As the Reynolds number decreases, viscous effects tend to attenuate the efficiency of the inviscid vortex tilting mechanism described above. This is why the bending of the surface vorticity distribution in the wake becomes weaker at $\mathrm{Re}=100$ [Figs. 8(g) and 8(i)]. At the same time, the boundary layers thicken, so that the flow near the lower edge of the vortical region in the near wake starts to interact significantly with the wall shear layer, just as it does in the unsheared case. This interaction strengthens as the Reynolds number decreases ( $\mathrm{Re}=10$ and 0.1$)$, making the situation combine effects of the wall-induced asymmetry with those of the shear-induced asymmetry within the wake itself. As the wall-induced and shear-induced lift mechanisms both result in a force directed toward $x>0$ if Sr is positive [Fig. 1(c)], they act together to yield an enhanced repulsive force in this case, as reflected in (10b). Conversely, they produce antagonistic effects if $\mathrm{Sr}$ is negative [Fig. 1(d)], which yields a reduced transverse force. Regarding viscous effects, the mechanisms described above indicate that the wall interaction tends to reduce the spanwise vorticity in the gap, while the excess surface vorticity resulting from the presence of the shear tends to enhance (resp. reduce) it when $\mathrm{Sr}$ is positive (resp. negative). The two effects just do the opposite on the free side of the bubble. For this reason, the top-bottom asymmetry of the $\omega_{y}$-vorticity distribution existing in the unsheared case is lowered (enhanced) by the shear if the bubble lags (resp. leads) the fluid. This is why the drag is decreased (resp. increased) by the shear for positive (resp. negative) Sr, as (10a) confirms.

\section{HYDRODYNAMIC FORCES ON THE BUBBLE}

In this section, we discuss variations of the drag and lift forces on the bubble revealed by numerical data in the light of the basic mechanisms described in Sec. V. Most of these data cover the range $0.1 \leqslant \operatorname{Re} \leqslant 500,|\mathrm{Sr}| \leqslant 0.5$, but some correspond to higher Reynolds number or shear rate. We found such larger values useful to determine the dependence of several contributions with respect to $\mathrm{Re}, \mathrm{Sr}$, and $L_{\mathrm{R}}$ in the high-Reynolds-number range. Note that, following earlier studies aimed at revealing fundamental mechanisms rather that sticking to specific experimental conditions, we vary arbitrarily $\mathrm{Re}, \mathrm{Sr}$, and $L_{\mathrm{R}}$ without considering whether or not a real bubble would remain approximately spherical in a given fluid under such conditions. In all cases, we make use of the numerical data to derive semiempirical models valid in either the low-to-moderate or moderate-to-large Re range. Then we combine these models to propose empirical fits valid at arbitrary Reynolds number.

\section{A. Fluid at rest at infinity}

\section{Drag}

The validity of the low-Re approximation for the wall-induced drag correction was confirmed during the validation of the numerical approach [see (A1a) and Fig. 21(a) in the Appendix]. Hence the primary focus here is on the behavior of the drag coefficient $C_{\mathrm{D} u}^{\mathrm{W}}\left(\mathrm{Re}, L_{\mathrm{R}}\right)$ at moderate-to-high Re. To appreciate the modification of the drag force due to the wall influence, we compare $C_{\mathrm{D} u}^{\mathrm{W}}$ to its counterpart in an unbounded flow, $C_{\mathrm{D} 0}^{\mathrm{U}}$. The relative drag change $\Delta C_{\mathrm{D} u}^{\mathrm{W}}=\left(C_{\mathrm{D} u}^{\mathrm{W}}-C_{\mathrm{D} 0}^{\mathrm{U}}\right) / C_{\mathrm{D} 0}^{\mathrm{U}}$ is plotted in Fig. 11(a). Beyond $\mathrm{Re}=100$, this figure reveals a clear increase, with both the Reynolds number and the inverse of the separation distance, $L_{\mathrm{R}}^{-1}$, of the relative influence of the wall on the drag. In that range of Reynolds number, the relative drag increase becomes significant only for $L_{\mathrm{R}} \lesssim 2$. We are not aware of any theoretical prediction available for $\Delta C_{\mathrm{D} u}^{\mathrm{W}}$ in the limit of very large Reynolds number. In contrast, the case of a spherical bubble rising along the axis of a circular pipe was considered in Ref. [59], where it was established asymptotically (for both $L_{\mathrm{R}} \gg 1$ and $\operatorname{Re} \gg 1$ ) 


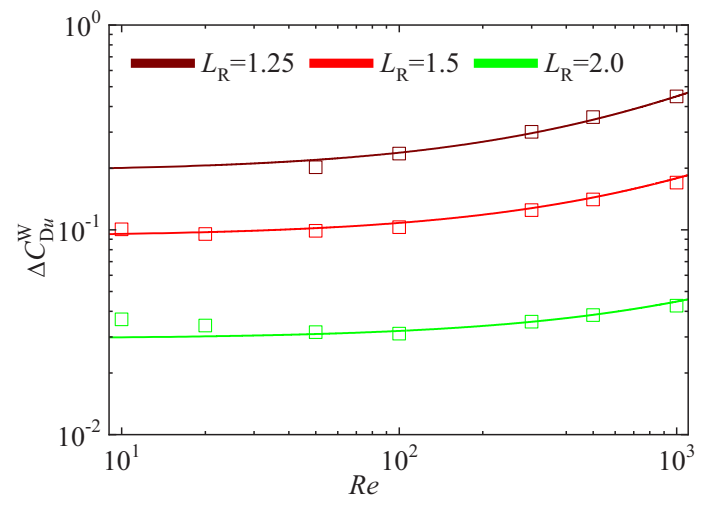

(a)

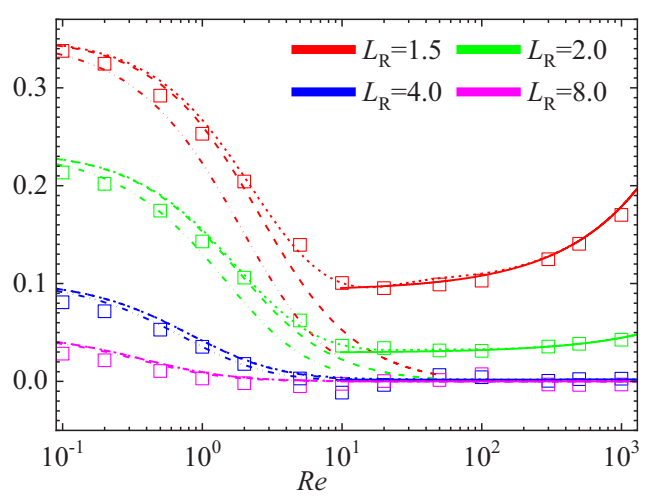

(b)

FIG. 11. Relative wall-induced drag correction $\Delta C_{\mathrm{D} u}^{\mathrm{W}}$ for a bubble translating parallel to a wall in a fluid at rest. (a) High-Re regime; (b) entire Re range investigated numerically. Symbols: numerical data; solid lines: high-Re expression (20); dash-dotted lines: low-Re expression (A1a); dashed lines: low-to-moderate-Re expression (22); dotted lines: composite fit (23).

that $\Delta C_{\mathrm{D} u}^{\mathrm{W}} \approx 1.6 L_{\mathrm{R}}^{-3}+0.2 \mathrm{Re}^{1 / 2} L_{\mathrm{R}}^{-9 / 2}$. The $L_{\mathrm{R}}^{-3}$-drag increase is a confinement effect, the vanishing of the fluid normal velocity at the pipe wall enhancing the local strain in the flow disturbance throughout the fluid. The $\mathrm{Re}^{1 / 2} L_{\mathrm{R}}^{-9 / 2}$ increase results from the wall boundary layer within which the dominant velocity gradient varies as $\mathrm{Re}^{1 / 2}$. Due to the sharp decrease of the latter contribution with $L_{\mathrm{R}}$, its influence is significant for large enough Re only. Conversely, it eventually becomes dominant whatever $L_{\mathrm{R}}$ provided Re is large enough. Although the geometrical configuration considered here differs from that in Ref. [59], the physical mechanisms responsible for the drag increase remain qualitatively unchanged. For this reason, we sought an empirical expression for $\Delta C_{\mathrm{D} u}^{\mathrm{W}}$ in the above form, adjusting both the prefactors and the exponents to the numerical data. Fitting the numerical results independently in the range $20 \leqslant \mathrm{Re} \leqslant 50$ (resp. $\operatorname{Re}>50$ ) to determine the Re-independent (resp. dependent) contribution, yielded

$$
\Delta C_{\mathrm{D} u}^{\mathrm{W}}(\operatorname{Re} \gg 1) \approx 0.47 L_{\mathrm{R}}^{-4}+5.5 \times 10^{-3} L_{\mathrm{R}}^{-6} \mathrm{Re}^{3 / 4} .
$$

Figure 11(a) shows that (20) compares well with the DNS data for Re $\gtrsim 50$. The surprising feature in (20) is that the separation distance and Reynolds number appear with exponents significantly different from those found in Ref. [59]. We carried out extensive tests to make sure that these findings are not due to an insufficient grid resolution. In particular we increased the number of nodes $N_{W}$ between the bubble and the wall up to 40 (resp. 60) for $L_{\mathrm{R}}=1.5$ (resp. 2), and the number of nodes $N_{\eta}$ in the radial direction up to 108 without observing any significant change in the drag and lift forces. This makes us confident that the results displayed in Fig. 11(a) are truly grid-independent. Also, since the relative drag variation induced by the wall boundary layer is still small for $L_{R}=2$ and 1.5 (it amounts to only $9 \%$ for $L_{\mathrm{R}}=1.5$ and $\mathrm{Re}=10^{3}$ ), we ran additional computations for $L_{\mathrm{R}}=1.25$, a shorter separation distance at which this effect is significantly larger. As the brown line in Fig. 11(a) reveals, (20) still closely follows the corresponding data, confirming that this fit is robust whatever $L_{R}$ within the range of Reynolds number we explored. This does not imply that it is asymptotically correct in the limit $\operatorname{Re} \rightarrow \infty$, and there are indications that it is not. Consider the situation in which the wall is replaced by a symmetry plane, which corresponds to the case of a pair of identical bubbles rising side by side. No boundary layer exists along the symmetry plane, and the drag modification in the limit $\operatorname{Re} \rightarrow \infty$ is then just a consequence of the nonpenetration condition across this plane. In the inviscid limit, this condition generates an $O\left(L_{\mathrm{R}}^{-3}\right)$ increase in the relative velocity $U_{\text {rel }}$, which in turn yields an $O\left(L_{\mathrm{R}}^{-3}\right)$ relative drag increase [48]. However, numerical data 
provided in Ref. [48] show that, for $L_{\mathrm{R}} \lesssim 1.5, \Delta C_{\mathrm{D} u}^{\mathrm{W}}$ still increases with the Reynolds number up to $\mathrm{Re} \approx 500$ before becoming Re-independent. Consequently the asymptotic regime in which $\Delta C_{\mathrm{D} u}^{\mathrm{W}}$ no longer depends on Re is reached only for very large Reynolds numbers. Here, as previously mentioned, the "Re-independent" contribution to (20) was determined from data corresponding to Reynolds numbers of some tens because this is the range where the wall boundary layer effect is still negligibly small. However, viscous effects are still present in this Re range, as the above example revealed. This is confirmed by Figs. 8(h) and 10(b), which show that, for $L_{\mathrm{R}} \leqslant 2$, the vortical region round the bubble directly interacts with the wall boundary layer at such moderate Reynolds numbers. This interaction is much weaker when $\mathrm{Re}=500$ [Figs. $8(\mathrm{k})$ and $10(\mathrm{e})$ ], suggesting that the ultimate behavior corresponding to $\operatorname{Re} \rightarrow \infty$ is reached only for $\operatorname{Re} \gtrsim 500$.

Figure 11(b) displays the relative drag increase throughout the range of Reynolds number explored in the simulations. Examination of the low-to-moderate-Re range indicates that (A1a) underpredicts the drag increase at small separations as soon as the Reynolds number is beyond the range 0.2-0.5 [see also Fig. 21(a)]. Following Ref. [13], this prediction may be improved by noting that the drag increase at low-to-moderate-Re is proportional to the square of the maximum vorticity at the bubble surface. Variations of this quantity with the Reynolds number are empirically expressed by the fitting function $b(\mathrm{Re})$ in $(16 \mathrm{a})$. However, we found that a more accurate fit of the DNS data reported in Ref. [50] is provided by

$$
b(\operatorname{Re})=1+\tanh \left(0.012 \mathrm{Re}^{0.8}\right)+\tanh \left(0.07 \mathrm{Re}^{0.8}\right) .
$$

Making use of (21), we then extend (A1a) toward moderate Reynolds numbers by expressing the drag increase in the form

$$
\Delta C_{\mathrm{D} u}^{\mathrm{W}}[\operatorname{Re}=O(1)] \approx f_{\mathrm{D}}^{\prime} b^{2}(\mathrm{Re}) \Delta C_{\mathrm{D} u}^{\mathrm{W}-\mathrm{in}},
$$

with $C_{\mathrm{D} u}^{\mathrm{W} \text {-in }}$ and $f_{\mathrm{D}}^{\prime}$ as given in (10a) and (12a), respectively. As Fig. 11(b) shows, this expression allows an accurate prediction of $\Delta C_{\mathrm{D} u}^{\mathrm{W}}$ up to $\mathrm{Re} \approx 2$. Obviously neither (22) nor (20) is accurate in the intermediate range $2 \lesssim \operatorname{Re} \lesssim 20$. Nevertheless, a simple fit may be obtained by combining empirically the above two models in the form

$$
\begin{aligned}
\Delta C_{\mathrm{D} u}^{\mathrm{W}}(\mathrm{Re}) & \approx \Delta C_{\mathrm{D} u}^{\mathrm{W}}[\mathrm{Re}=O(1)]+c_{D u \infty} \Delta C_{\mathrm{D} u}^{\mathrm{W}}(\operatorname{Re} \gg 1), \\
\text { with } \quad c_{\mathrm{D} u \infty} & =1-e^{-0.07 \operatorname{Re}} .
\end{aligned}
$$

As Fig. 11(b) shows, this composite expression correctly reproduces the computed drag variations throughout the entire range of Reynolds number.

\section{Lift}

Similar to wall-induced drag variations, the validity of the solution based on the combination of (10b) and (12b) for the transverse (lift) force coefficient in the low-but-finite-Re regime was confirmed during the validation of the numerical approach [see (A1b) and Fig. 21(b) in the Appendix]. For this reason, we first discuss the behavior of $C_{\mathrm{L} u}^{\mathrm{W}}\left(\mathrm{Re}, L_{\mathrm{R}}\right)$ in the high-Re range $10^{2} \leqslant \operatorname{Re} \leqslant 10^{3}$, before examining the variations of the transverse force at arbitrary Reynolds number. Figure 12(a) compares the numerical data obtained for $C_{\mathrm{L} u}^{\mathrm{W}}$ in that range with the irrotational prediction (17). The agreement is excellent as soon as the Reynolds number is large enough $(\operatorname{Re} \gtrsim 300)$ and the separation distance is small enough $\left(L_{\mathrm{R}} \lesssim 4\right)$. The viscous effect associated with the asymmetry of the boundary layer surrounding the bubble, which yields the correction (18), becomes significant at smaller Re, where it lowers the negative (i.e., attractive) transverse force. Numerical results also confirm the sharp decay of the transverse force when $L_{\mathrm{R}}$ increases, as predicted by (17), which makes the attractive effect negligibly small for dimensionless separations larger than a few units.

Figure 12(b) shows how $C_{\mathrm{L} u}^{\mathrm{W}}\left(\operatorname{Re}, L_{\mathrm{R}}\right)$ varies with the Reynolds number throughout the range $10^{-1} \leqslant \operatorname{Re} \leqslant 10^{3}$. In line with experiments reported in Ref. [6], the transverse force is found to switch from positive (i.e., repulsive) to negative (i.e., attractive) in the range $40 \leqslant \operatorname{Re} \leqslant 60$, 


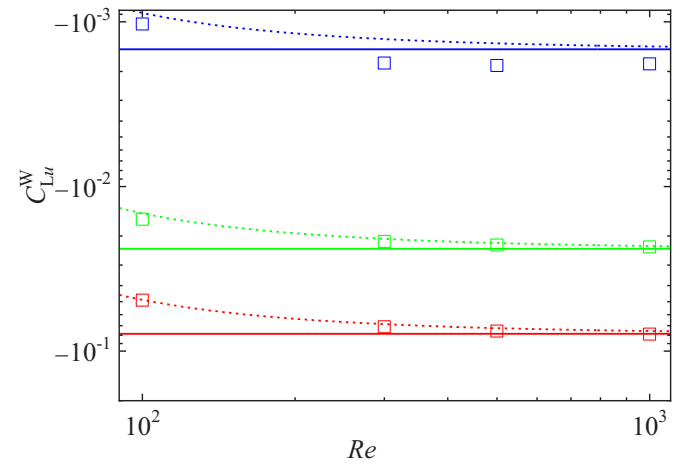

(a)

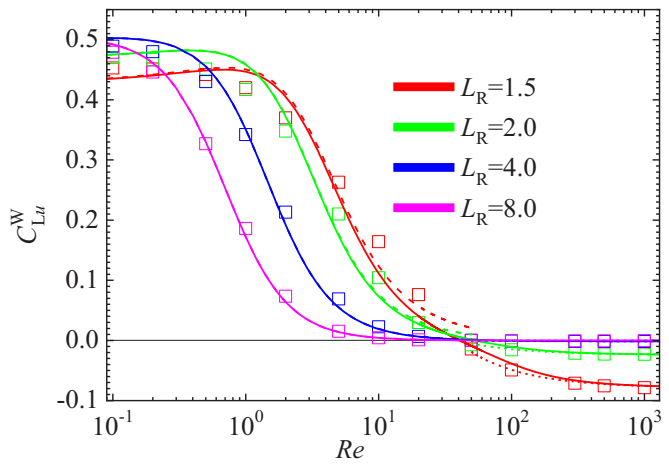

(b)

FIG. 12. Lift coefficient on a bubble translating parallel to a wall in a fluid at rest. (a) High-Reynoldsnumber range $10^{2} \leqslant \operatorname{Re} \leqslant 10^{3}$. Comparison of numerical results (symbols) with the potential flow prediction (17) (solid lines), and the high-Re prediction (18) including the $\mathrm{Re}^{-1}$ vortical contribution (dotted lines). (b) Complete Re range $10^{-1} \leqslant \operatorname{Re} \leqslant 10^{3}$. Comparison of numerical results (symbols) with the lowto-moderate-Re semiempirical model (24) (dashed lines), the high-Re prediction (18) (dotted lines), and the composite model (solid lines).

depending on $L_{\mathrm{R}}$; the smaller $L_{\mathrm{R}}$ the lower the critical Reynolds number at which $C_{\mathrm{L} u}^{\mathrm{W}}$ changes sign. At low Reynolds number, numerical data indicate that $C_{\mathrm{L} u}^{\mathrm{W}} \rightarrow 1 / 2$ for $L_{\mathrm{R}} \gtrsim 4$, in agreement with the asymptotic prediction (10b). Higher-order terms in (10b), which represent effects of the bubble finite size, somewhat lower the transverse force when the bubble gets very close to the wall, owing to the negative sign of the $L_{\mathrm{R}}^{-2}$ contribution; numerical results confirm this trend (see especially the data set corresponding to $L_{\mathrm{R}}=1.5$ ). However, the increase of $C_{\mathrm{L} u}^{\mathrm{W}}$ with increasing $L_{\mathrm{R}}$ at a given $\operatorname{Re}$ is not monotonic, as may be seen by spotting values of $L_{\mathrm{R}}$ for increasing values of $C_{\mathrm{L} u}^{\mathrm{W}}$ at $\mathrm{Re}=0.1$ and 1 . This is because, beyond a certain $L_{\mathrm{R}}$, the wall stands in the outer region of the disturbance, as illustrated in Fig. 3. Then, since $L_{u}=L_{\mathrm{R}} \mathrm{Re} / 2$, inertial effects due to the wall influence, which are at the root of the wall-induced lift force, start to decrease as $L_{\mathrm{R}}$ increases, in agreement with (12b). This is also the origin of the large decrease of the lift force with Re observed at every separation distance in the Reynolds number range where the flow disturbance gradually switches from the low-Re regime to the moderate-Re regime; e.g., in the range $1 \leqslant \mathrm{Re} \lesssim 30$ for $L_{\mathrm{R}}=1.5$ and $0.1 \leqslant \operatorname{Re} \lesssim 10$ for $L_{\mathrm{R}}=8$. In Ref. [6], the semiempirical expression (15) was proposed to estimate the transverse force for moderate separations and Reynolds numbers, typically $L_{\mathrm{R}} \gtrsim 2$ and $\mathrm{Re}=O(10)$. This expression accounts for the variations of the vorticity magnitude at the bubble surface as a function of Re through the empirical function $b(\mathrm{Re})$ given in (16a). In contrast, it does not account for the aforementioned finite-size effects which become important for small separations, as it makes use of the prediction for $C_{\mathrm{L} u}^{\mathrm{W}-\text { out }}(\mathrm{Re} \ll 1)$ resulting from $(11 \mathrm{~b})$, in which the bubble is treated as a point force. To improve this aspect, we heuristically replace $C_{\mathrm{L} u}^{\mathrm{W}-\text { out }}(\mathrm{Re} \ll 1)$ in $(15)$ by $C_{\mathrm{L} u}^{\mathrm{W}}(\operatorname{Re} \ll 1) \approx f_{\mathrm{L}}^{\prime} C_{\mathrm{L} u}^{\mathrm{W}-\text { in }}$ as given in $(\mathrm{A} 1 \mathrm{~b})$, so that $(15)$ becomes

$$
C_{\mathrm{L} u}^{\mathrm{W}}[\operatorname{Re}=O(1-10)] \approx b^{2}\left(L_{\mathrm{R}} / 3\right)^{g} f_{\mathrm{L}}^{\prime} C_{\mathrm{L} u}^{\mathrm{W}-\mathrm{in}},
$$

with $C_{\mathrm{L} u}^{\mathrm{W}-i n}, f_{\mathrm{L}}^{\prime}, b$, and $g$ as given in (10b), (12b), (21), and (16), respectively. The prediction of (24) [dashed lines in Fig. 12(b)] is found to agree well with the DNS data up to $\operatorname{Re} \approx 10$. For practical purpose, we sought an empirical composite model similar in essence to (19), capable of fitting the variations of $C_{\mathrm{L} u}^{\mathrm{W}}$ throughout the explored range of Reynolds number. In (19), the high-Re viscous contribution which makes $C_{\mathrm{L} u}^{\mathrm{W}}(\mathrm{Re} \gg 1)$ differ from $C_{\mathrm{L} u}^{\mathrm{W}}(\mathrm{Re} \rightarrow \infty)$ [see (17) and (18)] was not considered, since the experiments reported in Ref. [6] were all run at Reynolds numbers less than 100. However, as Fig. 12(a) shows, this viscous effect is significant for $\operatorname{Re}=O(100)$. We found that the simplest way in which the various contributions may be combined while respecting 


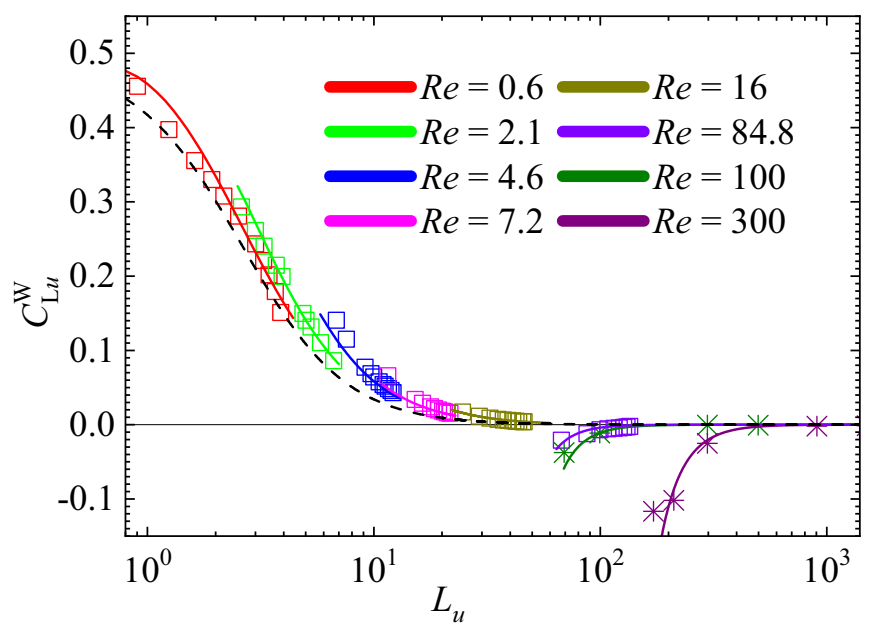

FIG. 13. Transverse force on a bubble translating parallel to a wall in a fluid at rest in the range $0.6 \leqslant$ $\operatorname{Re} \leqslant 300$ according to various sources. Solid line: composite model (25) involving the low-to-moderate-Re model (24)-(21); dashed line: low-but-finite-Re outer solution (12b); $\square$ : experimental data from [13] and [6]; $*$ : numerical data from Ref. [9].

the proper asymptotic behaviors and having a limited impact out of their own range of validity reads

$$
\begin{aligned}
C_{\mathrm{L} u}^{\mathrm{W}} & \approx C_{\mathrm{L} u}^{\mathrm{W}}[\operatorname{Re}=O(1-10)]+c_{T 1}\left[C_{\mathrm{L} u}^{\mathrm{W}}(\mathrm{Re} \rightarrow \infty)+c_{T 2} \operatorname{Re}^{-1} L_{\mathrm{R}}^{-4}\right], \\
\text { with } \quad c_{T 1}(\mathrm{Re}) & =1-e^{-0.22 \mathrm{Re}^{0.6}}, \quad c_{T 2}(\operatorname{Re})=15 \tanh (0.01 \mathrm{Re}) .
\end{aligned}
$$

As shown by the solid lines in Fig. 12(b), this empirical fit agrees well with the numerical data throughout the explored range of parameters.

To further test the capabilities of the above composite model, Fig. 13 compares its predictions with available numerical and experimental data taken from other sources. These data were obtained by considering bubbles with different diameters and changing the separation distance for each of them. This is why each series plotted in the figure corresponds to a fixed Reynolds number and varying $L_{u}=(\operatorname{Re} / 2) L_{\mathrm{R}}\left(L_{\mathrm{R}}\right.$ varies from 2.5 to 10 in the reported experiments, and from 1.2 to 20 in the computations). As expected, low-Re data approach the outer solution (12b) as $L_{u}$ becomes of $O(1)$ (consider especially the series corresponding to $\operatorname{Re}=0.6$ ). The transverse force decays more slowly than predicted by this solution when the Reynolds number is of $O(1-10)$. As discussed in Ref. [6], this is primarily an effect of the finite-Re increase of the vorticity produced at the bubble surface, which is reflected in the above fitting function $b(\mathrm{Re})$. Last, the transverse force changes sign for a critical Reynolds number of some tens, as it becomes dominated by the irrotational mechanism when viscous effects are small enough. The composite model (25), which improves over the original model (19) proposed in Ref. [6], is seen to properly capture these successive features.

\section{B. Linear shear flow}

We now perform the same type of analysis in the more complex situation where the effects of a constant shear combine with those of the wall.

\section{Drag}

Results for the drag change ratio $\Delta C_{\mathrm{D}}^{\mathrm{W}}=\left(C_{\mathrm{D}}^{\mathrm{W}}-C_{\mathrm{D} 0}^{\mathrm{U}}\right) / C_{\mathrm{D} 0}^{\mathrm{U}}$ obtained in the low-but-finite Reynolds-number regime, $0.1 \leqslant \mathrm{Re} \leqslant 2$, with a dimensionless shear rate $\mathrm{Sr}= \pm 0.5$ are plotted 


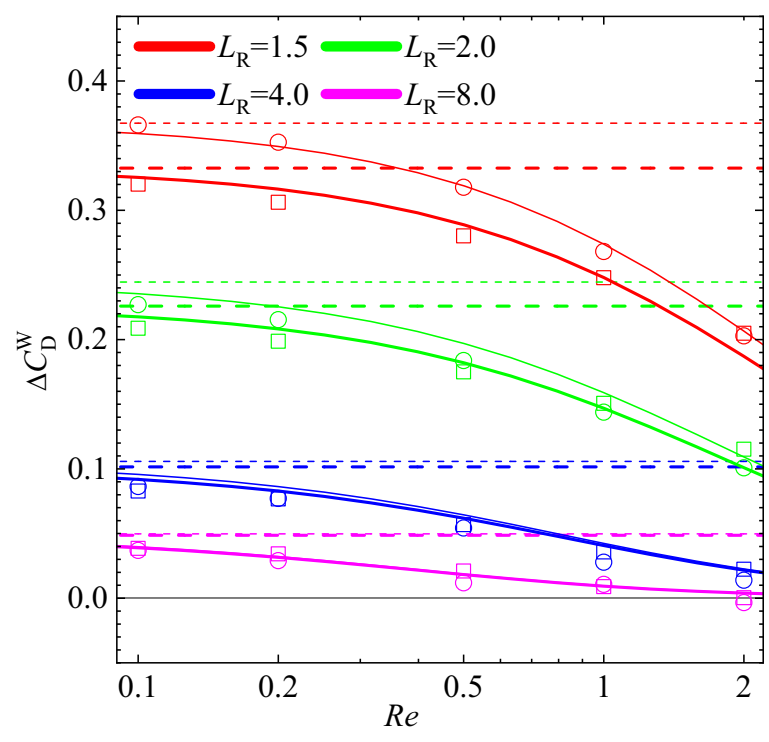

FIG. 14. Relative near-wall drag increase $\Delta C_{\mathrm{D}}^{\mathrm{W}}$ for a bubble translating parallel to a wall in a linear shear flow in the low-but-finite Reynolds-number regime for $\mathrm{Sr}= \pm 0.5$. Symbols: numerical results for $\mathrm{Sr}=0.5$ $(\square)$, and $\mathrm{Sr}=-0.5$ (०). Dashed lines: asymptotic prediction (10a) corresponding to conditions $L_{u} \ll 1, L_{\omega} \ll$ 1; solid lines: semiempirical model (26). Thick (resp. thin) lines correspond to positive (resp. negative) $\mathrm{Sr}$.

in Fig. 14 for different separation distances. Results found with smaller shear rates behave similarly. Clearly, the shear-induced drag modification is negligibly small compared with that due to the wall effect when $L_{R}$ exceeds a few units. For the smallest two separations, the shear is found to increase (resp. reduce) the drag when the bubble leads (resp. lags) the fluid, in line with the qualitative discussion provided at the end of Sec. V. The solution (10a) assuming that the wall stands in the inner region of the disturbance shows good agreement with numerical results at $\operatorname{Re}=0.1$ but, not surprisingly, overestimates them at higher Reynolds number. No explicit counterpart of the theoretical solution (11a) exists when the shear is nonzero and the wall stands in the outer region of the disturbance. However, the relative influence of the shear is always small under the conditions considered here (at $\mathrm{Re}=0.1$, the relative variation of $\Delta C_{\mathrm{D}}^{\mathrm{W}}$ is approximately $8 \%$ for $\mathrm{Sr}= \pm 0.5$ compared to the unsheared case $\mathrm{Sr}=0$ ). For this reason, we merely duplicate empirically the arguments employed in the unsheared configuration to derive (A1a) and (22), in order to obtain an estimate of the drag variation valid up to Reynolds numbers of $O(1)$. This yields

$$
\Delta C_{\mathrm{D}}^{\mathrm{W}}(\operatorname{Re}=O(1)) \approx f_{\mathrm{D}}^{\prime}\left(L_{u}\right) b^{2}(\operatorname{Re}) \Delta C_{\mathrm{D}}^{\mathrm{W}-\mathrm{in}},
$$

with $\Delta C_{\mathrm{D}}^{\mathrm{W}-\mathrm{in}}, f_{\mathrm{D}}^{\prime}\left(L_{u}\right)$, and $b(\mathrm{Re})$ as given in (10a), (12), and (21), respectively. As the solid lines in Fig. 14 show, the corresponding predictions properly follow the numerical data up to $\operatorname{Re} \approx 2$.

To analyze results obtained in the moderate-to-high-Re range, we first consider the unbounded configuration in which the generation of the lift force is dominated by the inviscid vortex tilting mechanism described in Sec. V. Figure 15 shows how the relative drag change $\Delta C_{\mathrm{D} \omega}^{\mathrm{U}}(\mathrm{Re}, \mathrm{Sr})=$ $\left[C_{\mathrm{D} \omega}^{\mathrm{U}}(\mathrm{Re}, \mathrm{Sr})-C_{\mathrm{D} 0}^{\mathrm{U}}(\mathrm{Re})\right] / C_{\mathrm{D} 0}^{\mathrm{U}}(\mathrm{Re})$ varies with both the Reynolds number and the dimensionless shear rate. Symmetry considerations imply that $\Delta C_{\mathrm{D} \omega}^{\mathrm{U}}(\mathrm{Re}, \mathrm{Sr})$ cannot depend on the sign of $\mathrm{Sr}$, which numerical results confirm. If the carrying flow were irrotational, one would expect the relative drag increase to be proportional to $\mathrm{Sr}^{2}$ and independent of $\mathrm{Re}$ in the limit $\mathrm{Re} \rightarrow \infty$, since boundary layer effects are negligibly small in this limit. This may be confirmed by evaluating theoretically the drag experienced by a spherical bubble translating in a pure axisymmetric straining flow of the form $\boldsymbol{u}_{\infty}=-\frac{\gamma}{2}\left(x \boldsymbol{e}_{x}+y \boldsymbol{e}_{y}\right)+(\gamma z-V) \boldsymbol{e}_{z}$. As there is no wake in that case in the limit $\operatorname{Re} \rightarrow$ 


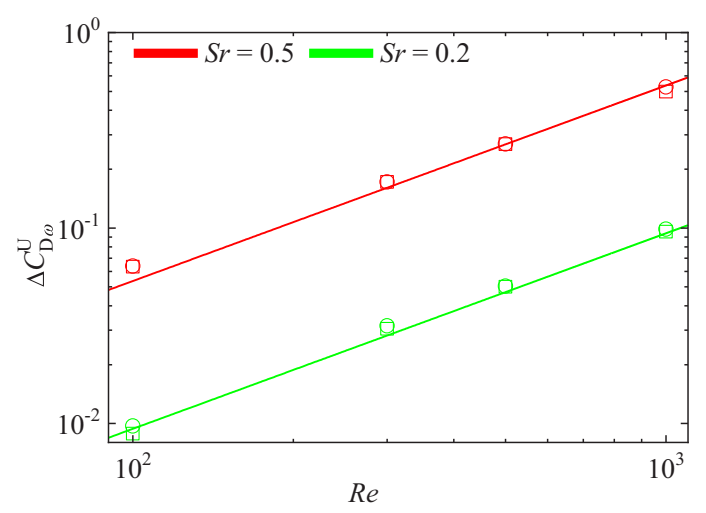

(a)

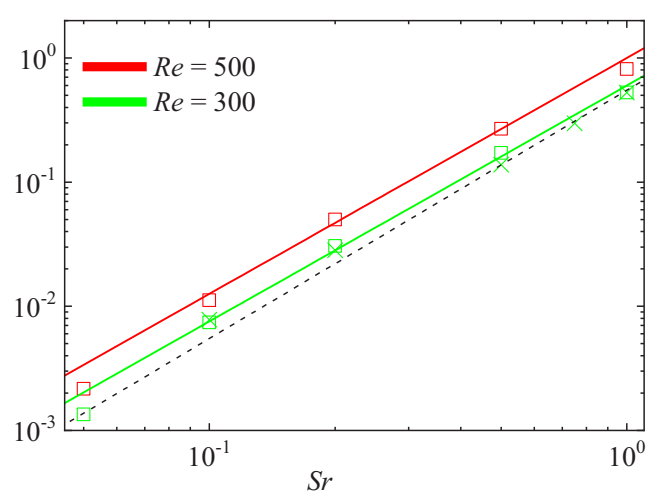

(b)

FIG. 15. Relative drag variation $\Delta C_{\mathrm{D} \omega}^{\mathrm{U}}$ for a bubble translating at moderate-to-high $\mathrm{Re}$ in an unbounded shear flow, with reference to the drag in a uniform stream. (a) Influence of the Reynolds number; (b) influence of the shear rate. (a) Numerical results for $\mathrm{Sr}>0$ ( $\square$ ), and $\mathrm{Sr}<0$ (०). (b) Numerical results for $\operatorname{Re}=500$ $(\square)$, and $\operatorname{Re}=300$ (०). Solid line: prediction of (27); dashed line: prediction of (9a) taken from Ref. [8]; $\times$ : numerical results from Ref. [8].

$\infty$, the work produced by the drag force exactly balances the dissipation in the entire flow [58]. Evaluating this dissipation yields $\Delta C_{\mathrm{D} \omega}^{\mathrm{U}}(\mathrm{Re} \rightarrow \infty, \mathrm{Sr})=\frac{2}{9} \mathrm{Sr}^{2}$, with $\mathrm{Sr}=\gamma d /|V|$. The situation is drastically different in the linear shear flow of interest here, since the disturbance is not irrotational and a wake made of the two counterrotating trailing vortices discussed in Sec. V extends to infinity downstream of the bubble. This configuration is similar to that of a wing of finite span, on which the trailing vortices are known to produce a lift-induced drag force proportional to the square of the lift force [58]. This additional drag is a second-order effect due to the interaction between the upstream vorticity and the vortex-induced velocity disturbance induced by the trailing vortices, here proportional to $\gamma d$. Consequently, it is proportional to $\rho d^{4} \gamma^{2}$, yielding an increase in the drag force proportional to $\mathrm{Sr}^{2} \rho d^{2} \mathrm{U}_{\text {rel }}^{2}$, hence a relative drag variation $\Delta C_{\mathrm{D} \omega}^{\mathrm{U}}$ proportional to $\mathrm{Sr}^{2} \mathrm{Re}$ since $C_{\mathrm{D} 0}^{\mathrm{U}} \propto R e^{-1}$ in this regime. Based on this analogy, we sought a fit of the results displayed in Fig. 15 in the form $\Delta C_{\mathrm{D} \omega}^{\mathrm{U}} \propto|\mathrm{Sr}|^{m} \mathrm{Re}^{n}$ and obtained

$$
\Delta C_{\mathrm{D} \omega}^{\mathrm{U}}(\operatorname{Re} \gg 1) \approx 2 \times 10^{-3}|\mathrm{Sr}|^{1.9} \mathrm{Re},
$$

which is seen to properly capture the trends revealed by numerical results. The fact that the $\mathrm{Sr}$ exponent in (27) is slightly lower than predicted in the inviscid lift-induced drag scenario is presumably a finite-Reynolds number effect that subsists in the limited Re range considered in present simulations.

Now we need to evaluate how much the above results are altered when the bubble gets close to the wall. Based on Figs. 8(j) and 8(1), one expects this alteration to be fairly weak, since the wall boundary layer is thin in the considered Re range, and to depend on the sign of $\mathrm{Sr}$, owing to the bending of the bubble wake towards (resp. away from) the wall for positive (resp. negative) Sr. To clarify the magnitude of the wall-shear interaction, we computed the difference between the actual drag variation in the near-wall configuration, $\Delta C_{\mathrm{D}}^{\mathrm{W}}\left(\mathrm{Re}, \mathrm{Sr}, L_{\mathrm{R}}\right)$, and the sum $\Delta C_{\mathrm{Du}}^{\mathrm{W}}\left(R e, L_{\mathrm{R}}\right)+\Delta C_{\mathrm{D} \omega}^{\mathrm{U}}(\mathrm{Re}, \mathrm{Sr})$ of the two contributions discussed above. This difference, say, $\Delta C_{\mathrm{D} \omega}^{\mathrm{W} \omega}(\operatorname{Re} \gg 1)$, is displayed in Fig. 16. The results show that it is negligibly small whatever the Reynolds number and for both shear rates for $L_{\mathrm{R}} \geqslant 2$. In addition to $L_{\mathrm{R}}=1.5$, we ran computations for $L_{\mathrm{R}}=1.25$ in order to determine the sharp decrease of $\Delta C_{\mathrm{D} \omega}^{\mathrm{W}-\mathrm{U}}(\operatorname{Re} \gg 1)$ as the separation increases. Figure 16 indicates that $\Delta C_{\mathrm{D} \omega}^{\mathrm{W}-\mathrm{U}}(\mathrm{Re} \gg 1)$ grows linearly with $\mathrm{Sr}$ and is positive (resp. negative) when the bubble lags (resp. leads) the fluid. This was expected on the basis of Figs. 8(j) and $8(1)$, since the interaction between the wake and the wall is stronger when $\mathrm{Sr}$ is positive, due to 


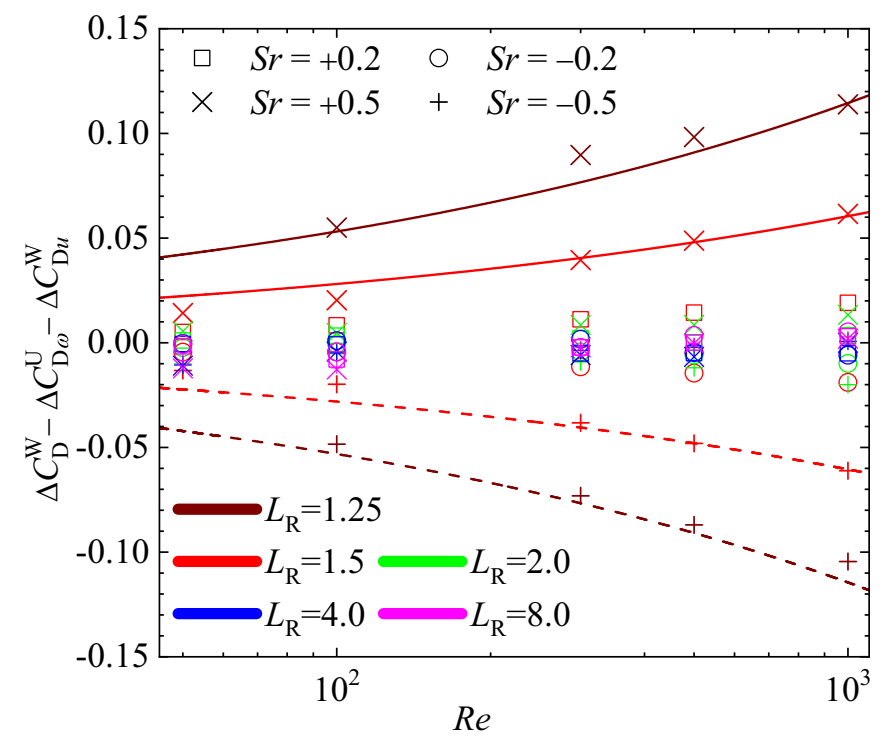

FIG. 16. Influence of the wall-shear interaction on the relative drag increase for $\mathrm{Re} \geqslant 100$. Symbols: numerical values of the difference between the relative drag variation in the presence of wall and shear $\left(\Delta C_{\mathrm{D}}^{\mathrm{W}}\right)$, and the sum $\Delta C_{\mathrm{D} u}^{\mathrm{W}}+\Delta C_{\mathrm{D} \omega}^{\mathrm{U}}$ of the relative drag variation in the presence of the wall for $\mathrm{Sr}=0\left(\Delta C_{\mathrm{D} u}^{\mathrm{W}}\right)$, and the relative drag variation in an unbounded shear flow $\left(\Delta C_{\mathrm{D} \omega}^{\mathrm{U}}\right)$. Solid (resp. dashed) lines: empirical expression (28) for $\mathrm{Sr}>0$ (resp. $\mathrm{Sr}<0)$.

the bending of the wake towards the wall. Based on DNS data, variations of $\Delta C_{\mathrm{D} \omega}^{\mathrm{W}-\mathrm{U}}(\operatorname{Re} \gg 1)$ with $L_{\mathrm{R}}$ and $\mathrm{Re}$ are found to be correctly fitted in the form

$$
\Delta C_{\mathrm{D} \omega}^{\mathrm{W}-\mathrm{U}}(\operatorname{Re} \gg 1) \approx 0.05 L_{\mathrm{R}}^{-7 / 2} \mathrm{SrRe}^{1 / 3} .
$$

Again, the above fit is grounded on a limited set of data obtained in the range $50 \leqslant \operatorname{Re} \leqslant 10^{3}$. Hence it is presumably not relevant in the limit $\operatorname{Re} \rightarrow \infty$. Nevertheless, up to $\operatorname{Re} \approx 10^{3}$, the above decomposition allows us to model the entire wall-induced drag correction in the moderate-to-highRe regime in the form

$$
\Delta C_{\mathrm{D}}^{\mathrm{W}}(\operatorname{Re} \gg 1) \approx \Delta C_{\mathrm{D} u}^{\mathrm{W}}(\operatorname{Re} \gg 1)+\Delta C_{\mathrm{D} \omega}^{\mathrm{U}}(\operatorname{Re} \gg 1)+\Delta C_{\mathrm{D} \omega}^{\mathrm{W}-\mathrm{U}}(\operatorname{Re} \gg 1),
$$

where the three contributions in the right-hand side are provided by (20), (27), and (28), respectively.

Figure 17 finally presents all numerical results obtained throughout the range $0.1 \leqslant \operatorname{Re} \leqslant 10^{3}$ together with the corresponding predictions based on the semiempirical expressions established above. Comparing the two panels, which differ only by the magnitude of $\mathrm{Sr}$, reveals that the shear has only a secondary influence on the drag increase up to Reynolds numbers of $O\left(10^{2}\right)$. In that range, shear-induced drag variations do not exceed $10 \%$ of the total drag increase for $\mathrm{Sr}= \pm 0.5$. This is in contrast with the behavior observed at higher Reynolds number, where $\Delta C_{\mathrm{D}}^{\mathrm{W}}(\operatorname{Re}=500)$ is found to be twice as large for $\mathrm{Sr}= \pm 0.5$ than for $\mathrm{Sr}= \pm 0.2$, owing to the large influence of the lift-induced-drag contribution (27). Clearly, the sign of Sr has only a small influence on $\Delta C_{\mathrm{D}}^{\mathrm{W}}$ at moderate-to-large $\mathrm{Re}$, as the shear is seen to increase the drag in all cases. In other words, the sign-dependent correction $\Delta C_{\mathrm{D} \omega}^{\mathrm{W}-\mathrm{U}}$ resulting from the wall-shear interaction is always small compared to lift-the induced-drag contribution $\Delta C_{\mathrm{D} \omega}^{\mathrm{U}}(\operatorname{Re} \gg 1)$. Interestingly, comparing $\Delta C_{\mathrm{D}}^{\mathrm{W}}$ in two flow configurations differing only by the sign of $\mathrm{Sr}$, it turns out that the drag increase is slightly larger at low Reynolds number when the bubble leads the fluid $(\mathrm{Sr}<0)$, in agreement with (10a). However, the reverse holds at large (and even moderate) Reynolds number. In a way similar to (23), one may seek an empirical expression combining linearly $\Delta C_{\mathrm{D}}^{\mathrm{W}}[\mathrm{Re}=O(1)]$ and $\Delta C_{\mathrm{D}}^{\mathrm{W}}(\operatorname{Re} \gg 1)$, as 


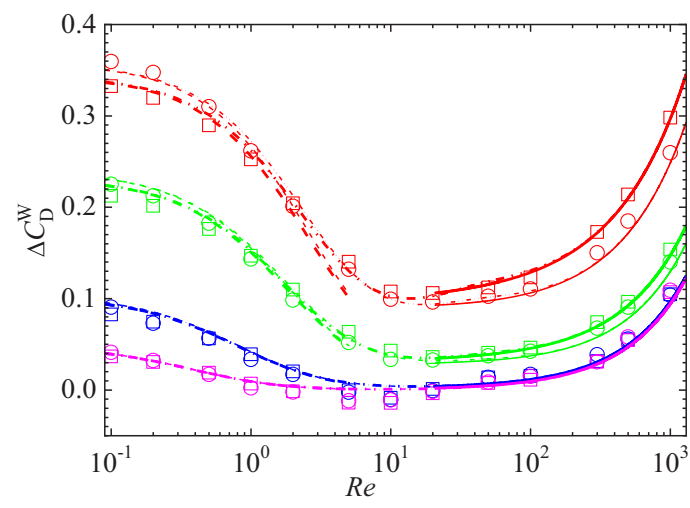

(a)

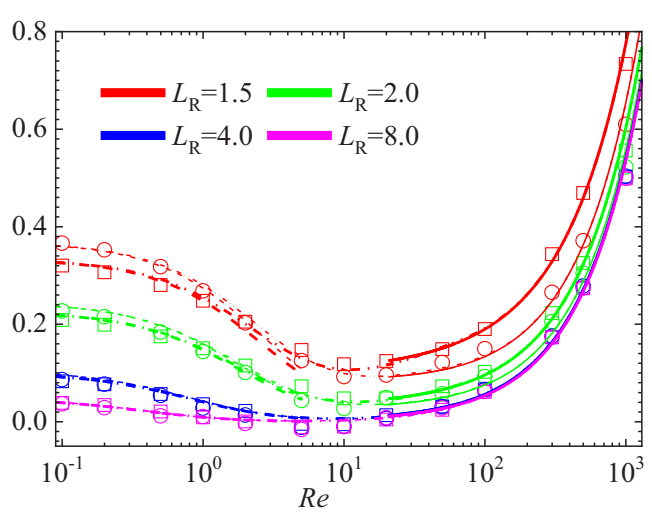

(b)

FIG. 17. Relative near-wall drag increase $\Delta C_{\mathrm{D}}^{\mathrm{W}}\left(\mathrm{Re}, \mathrm{Sr}, L_{\mathrm{R}}\right)$ for a bubble translating parallel to a wall in a linear shear flow throughout the Re range investigated numerically. (a) $\mathrm{Sr}= \pm 0.2$; (b) $\mathrm{Sr}= \pm 0.5$. $\square$ and $\circ$ : numerical data for $\mathrm{Sr}>0$ and $\mathrm{Sr}<0$, respectively. Thick (resp. thin) solid lines: high-Re expression (29) for positive (resp. negative) Sr; thick (resp. thin) dashed lines: low-Re expression (26) for positive (resp. negative) $\mathrm{Sr}$; thick (thin) dash-dotted lines: composite fit (30) for positive (resp. negative) Sr.

respectively given by (26) and (29), to improve the estimate of the drag increase in the intermediate range $2 \lesssim \operatorname{Re} \lesssim 20$. As the dash-dotted lines in Fig. 17 show, the composite expression

$$
\Delta C_{\mathrm{D}}^{\mathrm{W}}(\mathrm{Re}) \approx \Delta C_{\mathrm{D}}^{\mathrm{W}}[\mathrm{Re}=O(1)]+c_{D \omega \infty} \Delta C_{\mathrm{D}}^{\mathrm{W}}(\operatorname{Re} \gg 1) \quad \text { with } \quad c_{D \omega \infty}=1-e^{-0.07 R e}
$$

correctly fits the DNS data throughout the entire range of Reynolds number.

\section{Lift}

The computed lift coefficient $C_{\mathrm{L}}^{\mathrm{W}}\left(\mathrm{Re}, \mathrm{Sr}, L_{\mathrm{R}}\right)$ in the low-but-finite Reynolds-number regime is plotted in Fig. 18 for various normalized shear rates and separation distances. Numerical values corresponding to $L_{\mathrm{R}} \leqslant 2$ only slightly change with increasing $\mathrm{Re}$ up to $\mathrm{Re} \approx 0.5$, which provides an indication that the wall lies in the inner region of the disturbance in the corresponding $\left(\mathrm{Re}, L_{\mathrm{R}}\right)$ range. To properly interpret variations of $C_{\mathrm{L}}^{\mathrm{W}}$ at larger Reynolds numbers and separation distances, it is important to keep in mind that, since $\mathrm{Sr}$ is kept constant, increasing $\operatorname{Re}$ makes $\varepsilon=(\mathrm{Sr} / \mathrm{Re})^{1 / 2}$ decrease from left to right in each series. Consequently, for a given $L_{R}$, the larger Re the smaller the shear-induced lift force. The wall-induced contribution is also a decreasing function of the Reynolds number, since it depends directly on $L_{u}=\frac{1}{2} L_{\mathrm{R}} \mathrm{Re}$ and sharply decreases when $L_{u}$ becomes of $O(1)$ or larger in the shearless case, as shown in Fig. 6. Since the two contributions cooperate when the bubble lags the fluid, their variations as Re increases reinforce each other, making the total lift force decrease sharply as soon as the wall stands in the outer region (see, e.g., the data sets corresponding to $L_{\mathrm{R}}=4$ and 8 in the top left panel of Fig. 18). Conversely, these variations tend to compensate when the bubble leads the fluid, which mitigates the $C_{\mathrm{L}}^{\mathrm{W}}$ variations (consider the same two data sets in the bottom left panel, where these variations virtually cancel each other up to $\operatorname{Re} \approx 1$, resulting in an almost Re-independent total lift force).

Keeping $\mathrm{Re}$ and $\mathrm{Sr}$ constant, the influence of the separation distance is closely related to the successive behaviors of the shear-induced contribution as $L_{u}$ and $L_{\omega}$ increase. Indeed, as (10b) shows, this contribution grows linearly with $L_{\mathrm{R}}$ when the wall stands in the inner region. It grows more slowly when it stands in the outer region, until it reaches a constant value for large enough separations (this evolution is reflected in the $h_{L}$ function involved in (14); see also Figs. 4, 6, and 8 in Ref. [28]). Because of this gradual evolution, different behaviors of the total lift force are observed in Fig. 18. As far as the wall stands in the inner region or close to it, variations of $C_{\mathrm{L}}^{\mathrm{W}}$ with $L_{\mathrm{R}}$ are dominated by the nearly linear growth of the shear-induced contribution. Consequently, the lift 


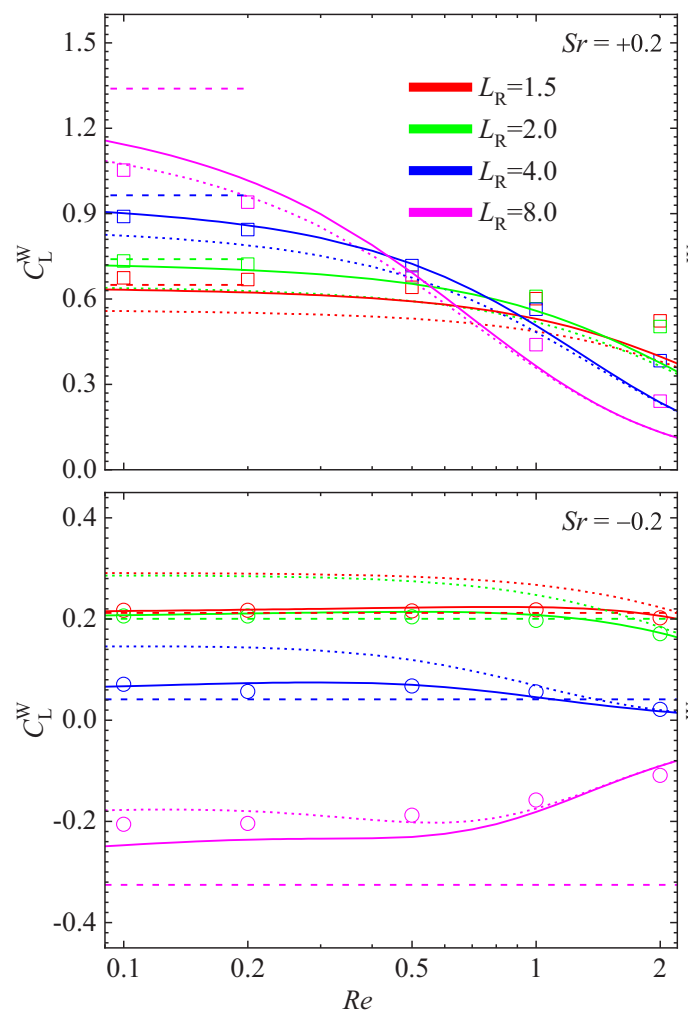

(a)

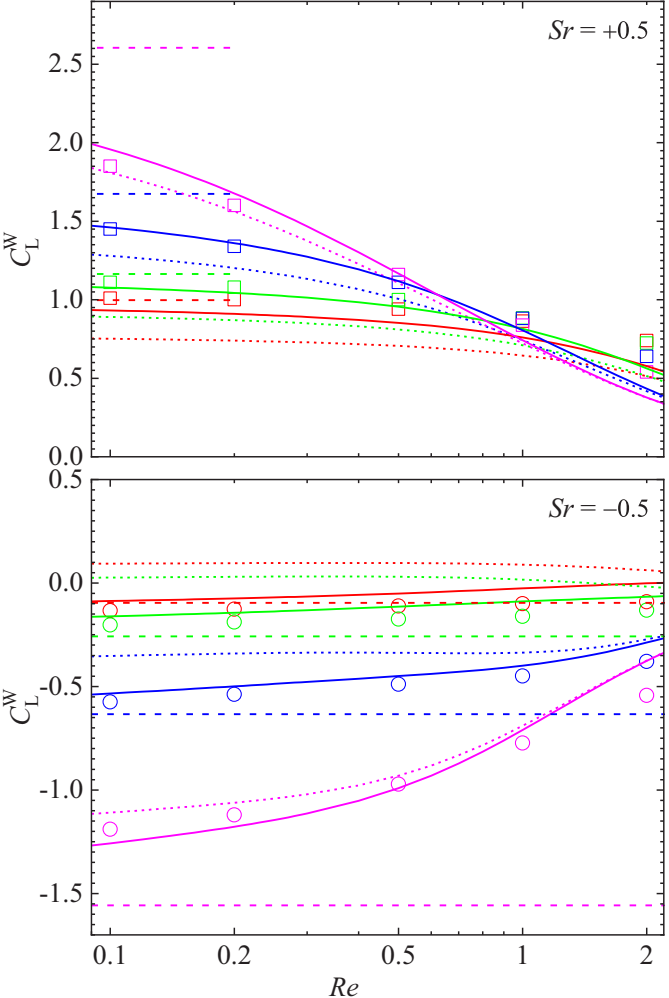

(b)

FIG. 18. Variations of the lift coefficient $C_{\mathrm{L}}^{\mathrm{W}}\left(\mathrm{Re}, \mathrm{Sr}, L_{\mathrm{R}}\right)$ for a bubble translating parallel to a wall in a linear shear flow in the low-but-finite Reynolds-number regime with (a) $\mathrm{Sr}= \pm 0.2$ and (b) $\mathrm{Sr}= \pm 0.5$. $\square$ (resp. ○): numerical data for $\mathrm{Sr}>0$ (resp. $\mathrm{Sr}<0$ ). Dashed lines: asymptotic prediction (10b) corresponding to conditions $L_{u} \ll 1, L_{\omega} \ll 1$; dotted lines: fit (13) of the asymptotic prediction corresponding to conditions $L_{u} \gg 1, L_{\omega} \gg 1$; solid lines: semiempirical model (31) taking into account finite-size effects.

force increases (resp. decreases, possibly changing sign) with increasing $L_{\mathrm{R}}$ when $\mathrm{Sr}>0$ (resp. $<0$ ). This is the behavior observed for $\mathrm{Re} \lesssim 0.5$ (resp. $\lesssim 0.8$ ) with $\mathrm{Sr}= \pm 0.2$ (resp. \pm 0.5 ). For larger Reynolds numbers, variations of the shear-induced contribution (which depends on $L_{\mathrm{R}}$ through $L_{u}$ and $L_{\omega}$ ) become weaker, so that the overall variation of the total lift force with $L_{\mathrm{R}}$ is mostly controlled by that of the wall-induced contribution (which depends on $L_{\mathrm{R}}$ through $L_{u}$ ). Since this contribution weakens dramatically as $L_{u}$ increases, the magnitude of the total lift force decreases, irrespective of its sign. When $\operatorname{Re}, \mathrm{Sr}$, and $L_{\mathrm{R}}$ (hence $L_{u}$ and $L_{\omega}$ ) are large enough, the wall-induced contribution becomes negligibly small, so that the total lift force reduces to the shear-induced contribution. In this situation, $C_{\mathrm{L}}^{\mathrm{W}}$ takes opposite values when the sign of $\mathrm{Sr}$ is reversed while $\mathrm{Re}$ and $L_{\mathrm{R}}$ are kept constant. This is what happens when the three parameters in Fig. 18 reach their maximum, i.e., $L_{\mathrm{R}}=8, \mathrm{Re}=2$ and $\mathrm{Sr}= \pm 0.5$. Indeed, the corresponding DNS data in the right two panels indicate $C_{\mathrm{L}}^{\mathrm{W}} \approx \pm 0.54$, depending on the sign of Sr. Interestingly, Fig. 18 of Ref. [8] indicates $C_{\mathrm{L} \omega}^{\mathrm{U}}(\mathrm{Re}=2, \mathrm{Sr}=0.5) \approx 0.56$ in an unbounded flow. Thus it can be concluded that the wall virtually no longer influences the lift force for the considered set of parameters, $L_{u}=8$ and $L_{\omega}=4$.

The asymptotic expression (10b) corresponding to situations in which the wall stands in the inner region of the disturbance (dashed lines in Fig. 18) closely predicts the computed lift force for small enough $L_{\mathrm{R}}$ and $\mathrm{Re}$, typically $L_{\mathrm{R}}<4$ and $\mathrm{Re} \leqslant 0.2$ for both shear rates. Not surprisingly, the agreement deteriorates as $L_{\mathrm{R}}$ or Re increases, owing to the increasing influence of inertial effects 
through $L_{u}$ and $L_{\omega}$. Overall, the approximate fit (13)-(14) of the asymptotic solution obtained in the case the wall stands in the outer region of the disturbance (dotted lines in Fig. 18) properly accounts for these effects. A closer look reveals that the corresponding prediction is in good agreement with the DNS data obtained for $L_{\mathrm{R}}=8$, at least up to $\mathrm{Re}=1$, but increasingly deviates from the computational results as $L_{\mathrm{R}}$ decreases. This is no surprise, as (14) is based on the outer solution of Ref. [28], which is valid only in the limit of large separations. Indeed, (14) approaches the leading-order expression of the asymptotic solution (10b) in the double limit $L_{u} \rightarrow 0$ and $L_{\omega} \rightarrow 0$, but does not account for the finite-size effects which affect both the wall-induced and the shear-induced contributions. This limitation may be overcome by modifying heuristically (13)-(14) in a way similar to that employed to obtain (24) in the unsheared case. That is, the outer solution $C_{\mathrm{L} u}^{\mathrm{W}-\text { out }}(\mathrm{Re} \ll 1)$ for the wall effect may be replaced in $(13)$ by the mixed expression $C_{\mathrm{L} u}^{\mathrm{W}}(\operatorname{Re} \ll 1) \approx$ $f_{L}^{\prime} C_{\mathrm{L} u}^{\mathrm{W}-\text { in }}$ [see (A1b)], and the $h_{\mathrm{L}}\left(L_{\omega}, \varepsilon\right)$ weighting function in (14) may be substituted by a function $h_{\mathrm{L}}^{\prime}\left(L_{\omega}, \varepsilon, L_{\mathrm{R}}\right)$ incorporating finite-size effects and tending towards $h_{\mathrm{L}}\left(L_{\omega}, \varepsilon\right)$ for large separations. Instead of (13)-(14) one then has

$$
\begin{aligned}
C_{\mathrm{L}}^{\mathrm{W}}(\mathrm{Re} \ll 1) & \approx f_{\mathrm{L}} C_{\mathrm{L} u}^{\mathrm{W}}(\operatorname{Re} \ll 1)+h_{\mathrm{L}}^{\prime} C_{\mathrm{L} \omega}^{\mathrm{U}}(\mathrm{Re} \ll 1), \\
\text { with } \quad h_{\mathrm{L}}^{\prime}\left(L_{\omega}, \varepsilon, L_{\mathrm{R}}\right) & =1-e^{-\frac{11}{96} \pi^{2} \frac{L_{\omega}}{J_{\mathrm{L}}(\varepsilon)}\left(1+\frac{9}{8} L_{\mathrm{R}}^{-1}-\frac{1271}{3520} L_{\mathrm{R}}^{-2}\right)},
\end{aligned}
$$

and $f_{L}\left(L_{\omega}, \varepsilon\right)$ still given by the first of (14). With these modifications, the semiempirical model (31) approaches the complete asymptotic solution (10b) when $L_{u} \rightarrow 0$ and $L_{\omega} \rightarrow 0$ (with the exception of the $\mathrm{Sr}^{2}$ term, usually much smaller than the $\mathrm{Sr}$ term as far as $\mathrm{Sr} \lesssim 1$ ), while the initial approximation (14) of the outer solution is recovered in the limit $L_{\mathrm{R}} \gg 1$. Compared to (13)-(14), the predictions of (31) (solid lines in Fig. 18) exhibit a significantly better agreement with numerical results when $L_{\mathrm{R}}$ and Re decrease.

Let us now examine the behavior of the lift force in the high-Reynolds-number range. At large enough separations, the wake past the bubble does not interact significantly with the wall, so that the lift coefficient must approach $C_{\mathrm{L} \omega}^{\mathrm{U}}(\operatorname{Re} \gg 1)$ as given in $(10 \mathrm{~b})$. When the bubble gets close to the wall, two distinct interaction mechanisms come into play. One is due to the bubble translation and would yield the attractive transverse force discussed in Sec. VI A 2 in the absence of shear. The other is due to the interaction of the shear-induced vortex structure past the bubble, especially the trailing vortices which dominate the wake, with the wall. This is similar to the ground effect known to increase the lift force on an aircraft flying very close to the ground. To quantify this second effect, the difference between the actual lift coefficient $C_{\mathrm{L}}^{\mathrm{W}}\left(\mathrm{Re}, \mathrm{Sr}, L_{\mathrm{R}}\right)$ and the sum $C_{\mathrm{L} \omega}^{\mathrm{U}}(\operatorname{Re} \gg$ $1)+C_{\mathrm{L} u}^{\mathrm{W}}(\operatorname{Re} \gg 1)$ [the latter as given in (18)] is plotted in Fig. 19(a). This figure shows that this difference, say, $\Delta C_{\mathrm{L}}^{\mathrm{W}}$, is small for $L_{\mathrm{R}}>2$ but becomes significant at smaller separations, sharply increasing as $L_{\mathrm{R}}$ reduces. As expected from the above analogy, $\Delta C_{\mathrm{L}}^{\mathrm{W}}$ is positive (resp. negative) for $\mathrm{Sr}>0($ resp. $\mathrm{Sr}<0)$ and is linearly proportional to Sr. Note that the sign of $\Delta C_{\mathrm{L}}^{\mathrm{W}}$ is in line with what may physically be expected from Fig. 8(j)-8(1), given the direction in which the wake bends for positive and negative $\mathrm{Sr}$, respectively. $\Delta C_{\mathrm{L}}^{\mathrm{W}}$ is also seen to be a slowly decreasing function of $\mathrm{Re}$, an effect which may be attributed to the wall boundary layer. Based on these observations, and fitting the numerical data of Fig. 19(a), we model the total lift coefficient in the form

$$
\begin{gathered}
C_{\mathrm{L}}^{\mathrm{W}}(\operatorname{Re} \gg 1)=C_{\mathrm{L} u}^{\mathrm{W}}(\operatorname{Re} \gg 1)+C_{\mathrm{L} \omega}^{\mathrm{U}}(\operatorname{Re} \gg 1)+\Delta C_{\mathrm{L}}^{\mathrm{W}}(\operatorname{Re} \gg 1), \\
\text { with } \frac{\Delta C_{\mathrm{L}}^{\mathrm{W}}(\operatorname{Re} \gg 1)}{C_{\mathrm{L} \omega}^{\mathrm{U}}(\operatorname{Re} \gg 1)}=I_{\mathrm{WS}} \approx a_{G} L_{\mathrm{R}}^{-7 / 2}\left(1+b_{G} \operatorname{Re}^{-1 / 2}\right), \text { where } a_{G} \approx 0.23 \text { and } b_{G} \approx 13 .
\end{gathered}
$$

The performance of this model is assessed in Fig. 19(b) by computing the difference $C_{\mathrm{L}}^{\mathrm{W}}\left(\operatorname{Re}, \mathrm{Sr}, L_{\mathrm{R}}\right)-C_{\mathrm{L}}^{\mathrm{W}}(\mathrm{Re} \gg 1)$ between the numerical value of the lift coefficient and the estimate provided by the model (32a) and (32b). This difference is normalized by $\left|C_{\mathrm{L} \omega}^{\mathrm{U}}\right|(\operatorname{Re} \gg 1)+$ $\left|C_{\mathrm{L} u}^{\mathrm{W}}\right|(\mathrm{Re} \gg 1)$, the sum of the estimated magnitudes of the two dominant contributions to the transverse force. The normalized difference, $\varepsilon_{\mathrm{WS}}$, is found to be only a few percent in all cases, confirming that the above model captures the main characteristics of this interaction mechanism. 


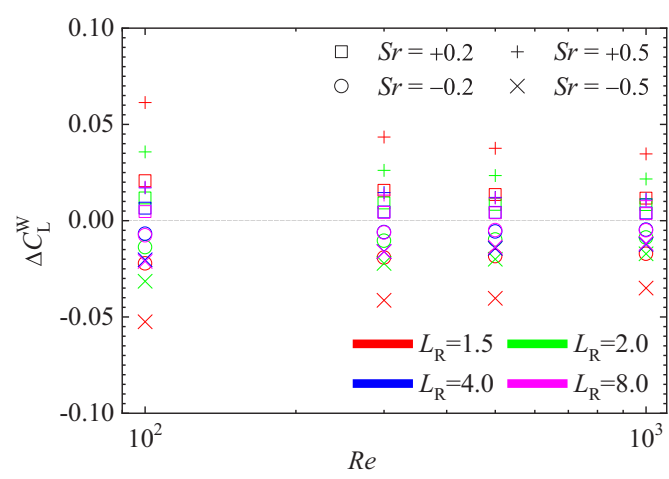

(a)

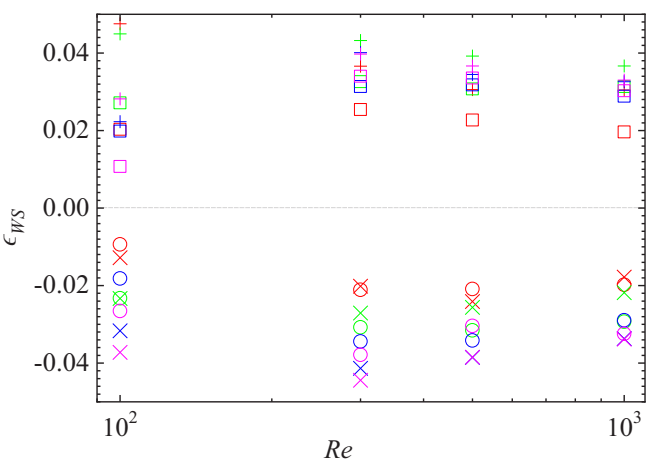

(b)

FIG. 19. (a) Effect of the shear-wall coupling on the lift force in the high-Reynolds-number regime. (a) Symbols: $\Delta C_{\mathrm{L}}^{\mathrm{W}}\left(\operatorname{Re}, \mathrm{Sr}, L_{\mathrm{R}}\right)=C_{\mathrm{L}}^{\mathrm{W}}\left(\operatorname{Re}, \mathrm{Sr}, L_{\mathrm{R}}\right)-C_{\mathrm{L} \omega}^{\mathrm{U}}(\operatorname{Re} \gg 1)-C_{\mathrm{L} u}^{\mathrm{W}}(\operatorname{Re} \gg 1)$. (b) Performance of the shear-wall coupling model (32a) and (32b) estimated through the normalized difference $\varepsilon_{\mathrm{WS}}=$ $\left[C_{\mathrm{L}}^{\mathrm{W}}\left(\operatorname{Re}, \mathrm{Sr}, L_{\mathrm{R}}\right)-C_{\mathrm{L}}^{\mathrm{W}}(\operatorname{Re} \gg 1)\right] /\left[\left|C_{\mathrm{L} \omega}^{\mathrm{U}}\right|(\operatorname{Re} \gg 1)+\left|C_{\mathrm{L} u}^{\mathrm{W}}\right|(\operatorname{Re} \gg 1)\right]$.

Figure 20 gathers the complete set of numerical results for the lift coefficient $C_{\mathrm{L}}^{\mathrm{W}}$ obtained in the range $0.1 \leqslant \operatorname{Re} \leqslant 10^{3}$. Clearly, most of the variations of the lift force with respect to both the Reynolds number and the separation distance take place for $\mathrm{Re} \lesssim 10$. At higher Reynolds numbers, discernible variations with the separation distance subsist only for $L_{\mathrm{R}} \leqslant 2$. For larger separations, the magnitude of the lift force becomes close to that found in an unbounded flow, as predicted by (9b). Interestingly, for $\mathrm{Sr}=-0.2$, the lift force changes sign at a critical Reynolds number in the range $3 \lesssim \operatorname{Re} \lesssim 15$ when the separation decreases from $L_{\mathrm{R}}=4$ to $L_{\mathrm{R}}=1.5$. This feature implies the existence of an equilibrium position of the bubble, resulting from a balance between the walland shear-induced lift components. Indeed, only the repulsive transverse force associated with the low-to-moderate-Re wall effect produces a positive transverse force in this case. This force may dominate the attractive shear-induced lift, provided $L_{\mathrm{R}}$ and $\mathrm{Sr}$ are small enough, which yields the above change of sign. No such feature is observed for $\mathrm{Sr}=-0.5$, since the negative shear-induced component is always dominant, even at small separations. Note that another equilibrium position, not encountered in Fig. 20, exists at large Reynolds number with weak positive shears and small separations. Indeed, balancing the dominant contributions in (17) and (9b) in the limit $\mathrm{Sr} \rightarrow 0^{+}$, $\mathrm{Re} \rightarrow \infty$ implies that the transverse force vanishes for $L_{\mathrm{R}} \approx(3 / 4)^{1 / 2} \mathrm{Sr}^{-1 / 4}$, i.e. $L_{\mathrm{R}} \approx 1.3$ for $\mathrm{Sr}=0.2$. The predictors of this equilibrium position may be discerned in the panel of Fig. 20 corresponding to $\mathrm{Sr}=0.2$, as the lift coefficient exhibits only small positive values at large Reynolds number, and these values are significantly decreasing with $L_{\mathrm{R}}$.

Predictions based on the low-Re model (31) and the high-Re model (32) are displayed in Fig. 20. These predictions are seen to properly follow numerical results for $\operatorname{Re} \lesssim 2$ and $\operatorname{Re} \gtrsim 20$, respectively. To fill the gap in which none of these models apply, we empirically combine linearly the composite model (25) for the wall-induced transverse force with a second composite model for the shear-induced contribution, providing a smooth variation from the low-Re expression (31) to the high-Re expression (32). Following this approach, the resulting composite fit reads

$$
C_{\mathrm{L}}^{\mathrm{W}}\left(\operatorname{Re}, \operatorname{Sr}, L_{\mathrm{R}}\right)=C_{\mathrm{L} u}^{\mathrm{W}}\left(\operatorname{Re}, \operatorname{Sr}, L_{\mathrm{R}}\right)+C_{\mathrm{L} \omega}^{\mathrm{W}}\left(\operatorname{Re}, \mathrm{Sr}, L_{\mathrm{R}}\right)
$$

with

$$
\begin{aligned}
& C_{\mathrm{L} u}^{\mathrm{W}}\left(\operatorname{Re}, \operatorname{Sr}, L_{\mathrm{R}}\right) \approx f_{\mathrm{L}} f_{\mathrm{L}}^{\prime} b^{2}\left(L_{\mathrm{R}} / 3\right)^{g} C_{\mathrm{L} u}^{\mathrm{W}-\mathrm{in}}+c_{T 1}\left[C_{\mathrm{L} u}^{\mathrm{W}}(\mathrm{Re} \rightarrow \infty)+c_{T 2} \operatorname{Re}^{-1} L_{\mathrm{R}}^{-4}\right], \\
& C_{\mathrm{L} \omega}^{\mathrm{W}}\left(\operatorname{Re}, \operatorname{Sr}, L_{\mathrm{R}}\right) \approx h_{\mathrm{L}}^{\prime} C_{\mathrm{L} \omega}^{\mathrm{U}}(\operatorname{Re} \ll 1)+c_{T 3}(\operatorname{Re})\left(1+I_{\mathrm{WS}}\right) C_{\mathrm{L} \omega}^{\mathrm{U}}(\operatorname{Re} \gg 1) \text { with } c_{T 3}(\operatorname{Re})=1-e^{-0.3 R e},
\end{aligned}
$$




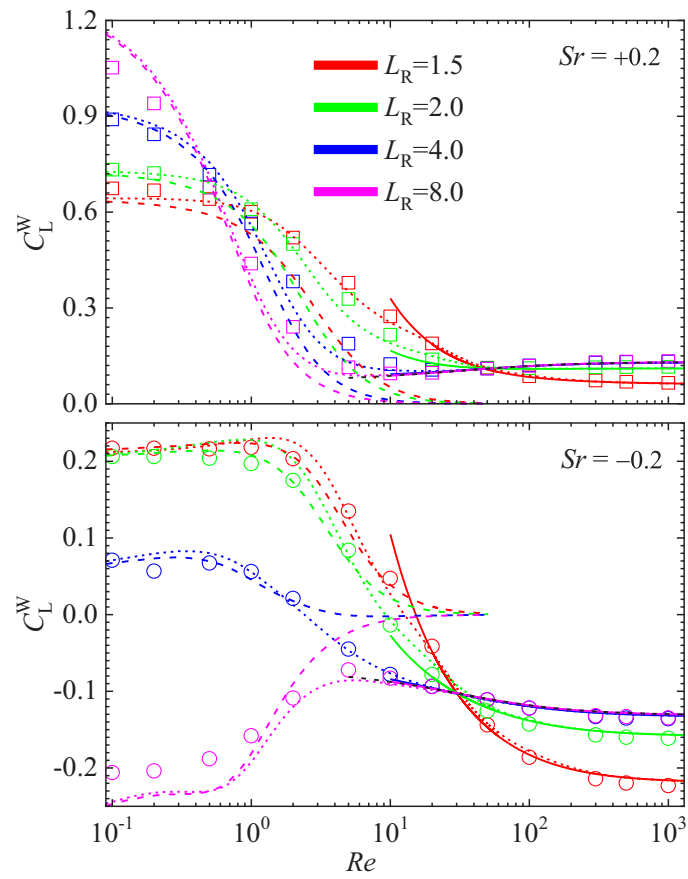

(a)
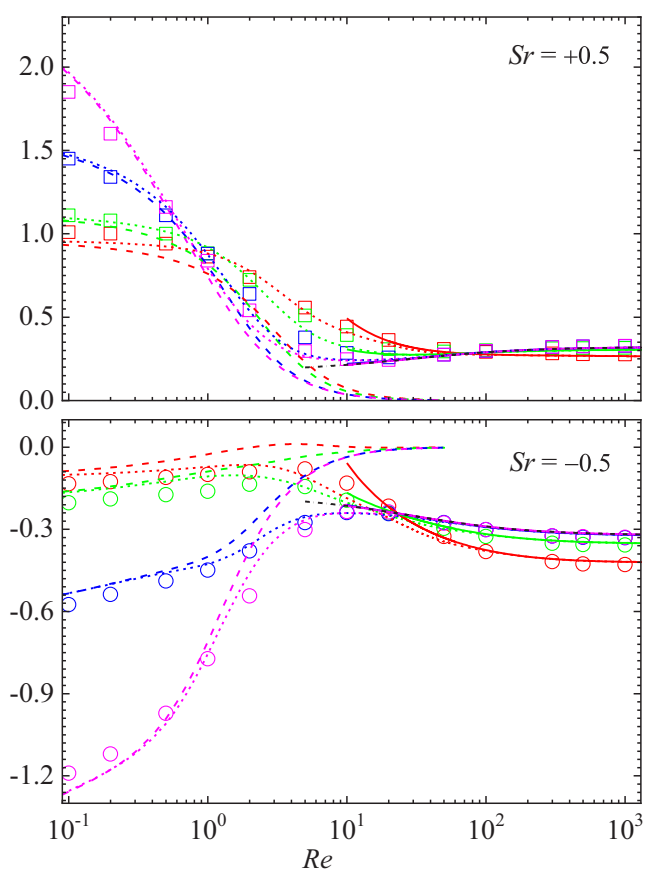

(b)

FIG. 20. Lift coefficient $C_{\mathrm{L}}^{\mathrm{W}}\left(\mathrm{Re}, \mathrm{Sr}, L_{\mathrm{R}}\right)$ for a bubble translating parallel to a wall in a linear shear flow throughout the Re range investigated numerically. (a) $\mathrm{Sr}= \pm 0.2$; (b) $\mathrm{Sr}= \pm 0.5$. $\square$ (resp. $\circ$ ): numerical data for $\mathrm{Sr}>0$ (resp. $\mathrm{Sr}<0$ ). Solid lines: high-Re prediction (32); dashed lines: low-Re prediction (31); dotted lines: composite expression (33); black dash-dotted line: moderate-to-high-Re behavior predicted by (9b) in an unbounded shear flow.

where $I_{\mathrm{WS}}$ is given in (32b). Figure 20 shows that this fit accurately follows the variations of the lift coefficient throughout the range of $\mathrm{Re}, \mathrm{Sr}$, and $L_{\mathrm{R}}$ covered by the simulations. Although it involves several empirical functions which depend on $L_{\mathrm{R}}, L_{u}, L_{\omega}$, and Re, it properly reduces to the relevant asymptotic expression in the various possible limits $\mathrm{Sr} \rightarrow 0, \mathrm{Re} \ll 1, \operatorname{Re} \gg 1$, and $L_{\mathrm{R}} \gg 1$.

\section{SUMMARY AND CONCLUSIONS}

In this paper we reported on a numerical study of the drag and lift forces acting on clean spherical bubbles rising along the wall of a wall-bounded linear shear flow. We considered flow configurations with the bubble either lagging or leading the fluid, so as to explore cooperative and antagonistic interactions between the wall- and shear-induced effects. With the selected range of wall distances, situations with the wall lying in either the inner or the outer region of the disturbance were both covered in the low-Reynolds-number regime. The selected Reynolds number range allowed us to consider situations dominated by viscous effects as well as nearly inviscid flows. In both asymptotic regimes, systematic comparisons with available analytical solutions were carried out.

For bubbles rising in a fluid at rest, low-Reynolds-number asymptotic solutions predict a drag increase due to the presence of the wall. Additionally, a repulsive transverse force takes place, due to the interaction between the wall and the wake resulting from the vorticity generated at the bubble surface by the shear-free condition. For a given separation distance, the magnitude of this repulsive force decreases with the Reynolds number when the wall lies in the outer region of the disturbance. At low-but-finite Reynolds number, both the drag increase and the transverse force are proportional to the square of the maximum vorticity at the bubble surface, which increases 
with the Reynolds number. Present computational results confirm these predictions, and support the model proposed in Ref. [6] for the prediction of the transverse force at low-to-moderate Reynolds number, albeit with some changes in the empirical coefficients. At higher Reynolds number, the boundary layers originating at the bubble surface and wall become thin and no longer interact significantly. This makes the flow in the gap almost irrotational, which results in an attractive transverse force as predicted by potential flow theory. However, an $O\left(\mathrm{Re}^{-1} L_{\mathrm{R}}^{-4}\right)$ viscous correction remains, and present results show that it significantly reduces the attractive transverse force for Reynolds numbers of $O\left(10^{2}\right)$. A drag increase also exists in the high-Reynolds-number regime but is significant only when the separation distance is small, typically $L_{R} \lesssim 2$. This increase results from two separate mechanisms. On the one hand there is a "confinement" effect imposed by the nonpenetration condition at the wall which induces an increase of the bubble relative velocity, hence a Re-independent relative drag increase when the Reynolds number is large enough. On the other hand, the wall boundary layer resulting from the no-slip condition provides a Re-dependent relative drag increase. In the limited Re range considered here $\left(\operatorname{Re} \leqslant 10^{3}\right)$, we found these two effects to be proportional to $L_{\mathrm{R}}^{-4}$ and $L_{\mathrm{R}}^{-6} \mathrm{Re}^{3 / 4}$, respectively. A point of caution, however, is that although these scalings accurately fit the numerical data in the moderate-to-large Re range investigated here, various arguments suggest that they do not hold in the limit $\operatorname{Re} \rightarrow \infty$.

Interactions between shear and wall effects have essentially been considered in the lowReynolds-number range in the available literature. In this regime, the solution of Ref. [4] valid when the wall stands in the inner region of the disturbance indicates a decrease (resp. an increase) of the drag due to shear effects when the bubble lags (resp. leads) the fluid, while the reverse holds for the transverse force. For this reason, the latter may switch from positive to negative at a given $L_{R}$ if the bubble leads the fluid and the magnitude of the shear is large enough. Computational results fully confirm these predictions, both qualitatively and quantitatively. When the wall stands in the outer region of the disturbance, they also confirm the corresponding theoretical predictions and their fit as proposed in Ref. [7]. Empirical expressions taking into account finite-size effects have been established to estimate the drag increase and the lift force irrespective of the wall position in the low-but-finite Reynolds-number regime. Comparison with numerical results indicates that they provide reliable predictions up to $\operatorname{Re}=O(1)$.

To disentangle the various effects at play in a wall-bounded shear flow at moderate-to-high Reynolds number, we first considered the well-documented unbounded configuration. The underlying idea was to superimpose linearly the corresponding results with those obtained in the wall-bounded situation in the absence of shear, in order to determine the strength of interactions not taken into account in this crude superposition scheme. In the inviscid limit, the lift and lift-induced drag forces on a bubble translating in an unbounded linear shear flow have the same origin as those experienced by a wing with a finite span. The tilting of the upstream spanwise vorticity past the body results in a horseshoe vortex in the wake, which, in the case of a sphere, yields a lift force with a coefficient of $\frac{2}{3} S r$ in the weak-shear limit [25]. Similarly, the drag increase due to the interaction between the upstream vorticity and the vortex-induced velocity disturbance in the wake causes a relative drag variation proportional to $\mathrm{Sr}^{2} \mathrm{Re}$. Computational results in the range $10^{2} \leqslant \operatorname{Re} \leqslant 10^{3}$ essentially confirm these predictions, although finite-Re effects are still present at such Reynolds numbers and slightly decrease the $\mathrm{Sr}$ exponent involved in the lift-induced drag correction. When a nearby wall is involved, the presence of the horseshoe vortex makes the wall-shear interaction dependent on the sign of Sr. Indeed, this vortex deflects the fluid located within the wake towards the wall or away from it, depending on whether the bubble lags or leads the fluid. Since the interaction between the wake and wall is stronger in the former case, the drag and total lift force are both increased (resp. decreased) when the bubble lags (resp. leads) the fluid. Computational results confirm the various aspects of this scenario. They indicate that, for moderate-to-large Reynolds numbers, say, $\mathrm{Re} \gtrsim 100$, the wall-shear interaction is significant only when the bubble moves very close to the wall, typically for $L_{\mathrm{R}} \leqslant 1.5$. As the Reynolds number decreases down to $\mathrm{Re} \approx 50$, viscous effects gradually inhibit the bending of the wake, reducing the overall repulsive (resp. attractive) force for $\mathrm{Sr}>0$ (resp. $\mathrm{Sr}<0)$. At somewhat lower Reynolds 
numbers, say, $5 \lesssim \operatorname{Re} \lesssim 50$, the two boundary layers thicken, strengthening the interaction between the wake and the wall shear layer if the separation distance is small. This results in an enhanced repulsive wall-induced contribution to the transverse force, which increases (resp. decreases) the magnitude of this force when $\mathrm{Sr}>0$ (resp. $\mathrm{Sr}<0$ ). For $\mathrm{Re} \lesssim 5$, the picture becomes more complex because several mechanisms combine. First, the Saffman and Oseen length scales grow as Re decreases in such a way that their ratio, $\varepsilon$, also grows. For a given $\mathrm{Sr}$, this variation tends to increase the shear-induced lift force in the unbounded configuration [see Fig. 23(b) below], because the role of the shear increases at the expense of that of the relative velocity in the transport of the disturbance downstream of the bubble. Then, in the wall-bounded configuration, the wake and wall shear layer are thick enough that they interpenetrate each other some distance downstream from the bubble, even for separation distances as large as $L_{\mathrm{R}}=8$. Keeping $\mathrm{Sr}$ and $\mathrm{Re}$ constant, the shear-induced lift mechanism experiences little change compared to the unbounded configuration if $L_{\mathrm{R}}$ is large, and essentially combines linearly with the wall-induced effect corresponding to the shearless configuration. Combining the above two arguments explains why, for a given $\mathrm{Re}$, the lift coefficient experiences large variations when $\mathrm{Sr}$ changes sign and $L_{\mathrm{R}}$ is large (consider the pink symbols corresponding to $L_{\mathrm{R}}=8$ in Fig. 20). However, as $L_{\mathrm{R}}$ is decreased, the wall tends to inhibit the tilting of the upstream vorticity past the bubble. Hence the shorter the separation distance, the smaller the fraction of the transverse force provided by the shear-induced contribution, so that this force becomes dominated by the repulsive wall-induced contribution. This is why, if the bubble leads the fluid, the shear has to be large for the transverse force to remain attractive (i.e., $C_{\mathrm{L}}^{\mathrm{W}}<0$ ) when $\mathrm{Re}$ is of order unity or less and $L_{\mathrm{R}}$ is small (red circles corresponding to $L_{\mathrm{R}}=1.5$ in Fig. 20).

Present numerical results help rationalize and quantify the various mechanisms reviewed above and the way they interact, provided the bubble surface is clean and its shape is approximately spherical. In general, the influence of deformation effects can drastically change the conclusions obtained with spherical bubbles. Let us just mention two examples in the case of an unbounded linear shear flow. It was shown experimentally [60] that the lateral migration of bubbles with equivalent diameters in the range 3-6 mm rising at moderate-to-large Reynolds number in highly viscous fluids (water-glycerin mixtures) changes sign when the bubble deformation is large enough. Similarly, the simulations in Ref. [44] revealed that the lift force experienced by spheroidal bubbles with a large enough oblateness rising at Reynolds numbers of some hundreds changes sign below a critical shear rate, $\mathrm{Sr}_{\mathrm{c}}$, which depends on both the Reynolds number and the bubble oblateness. The classical shear-induced mechanism illustrated in Fig. 9 dominates for $|\mathrm{Sr}|>\mathrm{Sr}_{\mathrm{c}}$, while wake instability provides a larger contribution of opposite sign for $|\mathrm{Sr}|<\mathrm{Sr}_{\mathrm{c}}$. In the lowRe regime, the theoretical predictions of Ref. [4] indicate that bubble deformation produces a repulsive wall-induced transverse force in the case where the fluid is at rest, thus cooperating with the inertia-induced transverse force. In contrast, in the case of a wall-bounded shear flow, the low-Re deformation-induced migration is towards the wall. Therefore, there is a critical deformation beyond which the total transverse force becomes attractive. It is clear that present results are of no help when such large deformations are encountered. In contrast they remain qualitatively valid for bubbles with small-to-moderate deformations. The high-Re situation was already discussed at the end of Sec. II. Following this discussion, results based on the spherical shape assumption are expected to be approximately valid up to $\operatorname{Re} \approx 350$, a situation where the bubble oblateness is approximately $20 \%$ [45]. At low Reynolds number, the ratio of the deformationinduced to inertia-induced contributions to the lateral force is governed by the Ohnesorge number, $\mathrm{Oh}=v(\rho / \sigma d)^{1 / 2}$, with $\sigma$ the surface tension. More precisely, this ratio is proportional to $\mathrm{Oh}^{2}$ and $\left(\mathrm{Oh} / L_{\mathrm{R}}\right)^{2}$ in the case of a wall-bounded linear shear flow and a wall-bounded stagnant fluid, respectively [see Eqs. (48) and (49) in Ref. [4]]. Considering again that predictions remain useful as far as the error on the total transverse force does not exceed $25 \%$, present results may be used up to $\mathrm{Oh} \approx 0.5$ in the case of a wall-bounded linear shear flow, and up to $\mathrm{Oh} \approx 0.75$ in a wall-bounded stagnant fluid, provided $L_{\mathrm{R}} \geqslant 1.5$. Most oils have a surface tension 3-4 times 
TABLE I. Effect of grid resolution on the drag and lift coefficients of a bubble translating close to a wall $\left(L_{\mathrm{R}}=2\right)$ in a stagnant fluid.

\begin{tabular}{cccccc}
\hline \hline & & $98 \times 54 \times 64$ & $98 \times 54 \times 128$ & $138 \times 78 \times 128$ & Analytical solution \\
\hline $\operatorname{Re}=0.1$ & $C_{\mathrm{D} u}^{\mathrm{W}}$ & 196.4 & 196.9 & 197.7 & 197.6 \\
& $C_{\mathrm{L} u}^{\mathrm{W}}$ & 0.470 & 0.468 & 0.469 & 0.467 \\
$\operatorname{Re}=100$ & $C_{\mathrm{D} u}^{\mathrm{W}}$ & 0.392 & 0.391 & 0.392 & - \\
& $C_{\mathrm{L} u}^{\mathrm{W}}$ & -0.016 & -0.016 & -0.016 & - \\
$\operatorname{Re}=500$ & $C_{\mathrm{D} u}^{\mathrm{W}}$ & 0.092 & 0.092 & 0.092 & - \\
& $C_{\mathrm{L} u}^{\mathrm{W}}$ & -0.023 & -0.023 & -0.023 & -0.024 \\
\hline \hline
\end{tabular}

smaller than pure water. Consequently, present low-Re results for a $1 \mathrm{~mm}$-diameter bubble may be considered reasonably accurate in oils with viscosities up to 70-80 times that of water.

From a practical point of view, present results provide several models for the drag correction and transverse force acting on nearly spherical bubbles. By incorporating relevant empirical finite-Re corrections, we proposed models grounded on asymptotic predictions established in the lowor high-Reynolds-number limit. Then we combined these models to obtain composite fits valid throughout the range of parameters explored numerically. Fits (23) and (24) may be used to predict the wall-induced drag correction and transverse force in a fluid at rest, respectively. Similarly, fits (30) and (33) and (34), which reduce to the former set for $\mathrm{Sr}=0$, apply in wall-bounded shear flows irrespective of the sign of the shear rate and relative fluid-bubble velocity. These models represent a significant improvement over current "point-particle" models of spherical bubbles which do not account for any wall-induced effect but are nevertheless routinely used to simulate laminar and turbulent wall-bounded bubbly flows. A similar investigation with rigid spheres, based on the same numerical approach and considering the same variety of flow configurations and regimes, is currently under way.

\section{ACKNOWLEDGMENTS}

This work was supported by the China Scholarship Council (CSC). We thank A. Pedrono for her intensive help and support with the JADIM code and the grid generator, and Dominique Legendre for his help with the grid generation technique.

\section{APPENDIX: VALIDATION AND COMPARISON WITH EXISTING RESULTS}

The capability of the JADIM code to accurately compute three-dimensional flows past bubbles and rigid bodies over a wide range of Reynolds number has already been proved in various contexts, (see e.g., Refs. $[8,16,61])$. The $(\xi, \eta, \varphi)$ grid system used here was also shown to be suitable to obtain accurate grid-independent results regarding the hydrodynamic interaction between two spherical bubbles rising side-by-side in a stagnant liquid [48]. Therefore, here we mainly check grid effects in wall-bounded flows and in an unbounded linear shear flow, respectively.

In a first series of tests, we computed the forces acting on a clean bubble translating in a wall-bounded stagnant fluid at finite Reynolds number. To obtain some insight into the adequacy of the grid resolution, this test was carried out first at $\operatorname{Re}=0.1,100$, and 500 with $L_{\mathrm{R}}=2$. Note that for $\operatorname{Re}=0.1$ and $L_{\mathrm{R}}=2, L_{u}=0.1$. Thus, the wall lies in the inner region of the disturbance and the solution (10) applies. The corresponding asymptotic drag and lift coefficients are 197.6 and 0.467 , respectively. At $\operatorname{Re}=500$, the resulting lift coefficient should be close to the irrotational prediction (17), $C_{\mathrm{L} u}^{\mathrm{W}}=-0.024$. Computational results obtained with three different grid resolutions are listed in Table I. The obtained drag and lift coefficients show good agreement with the analytical solution at $\operatorname{Re}=0.1$. At $\operatorname{Re}=500$, the deviation from (17) is approximately $4 \%$. This deviation is likely due to the viscous correction proportional to $\mathrm{Re}^{-1} L_{\mathrm{R}}^{-4}$ in (18). For all three Reynolds 


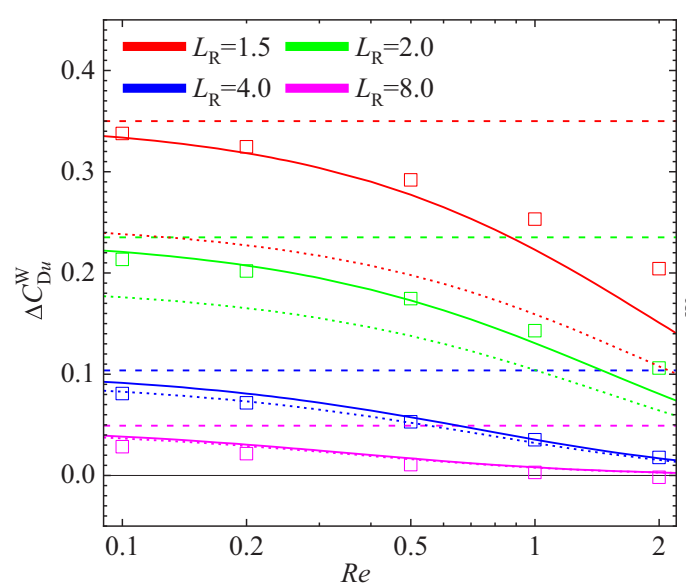

(a)

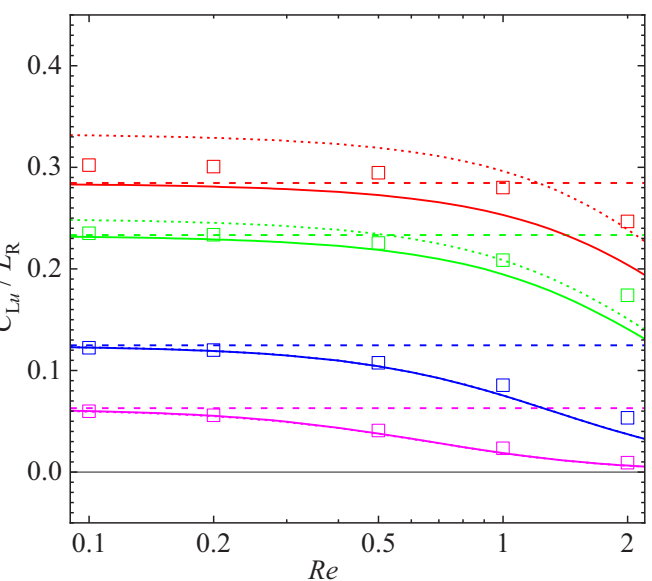

(b)

FIG. 21. Numerical predictions ( $\square$ ) for the wall-induced forces in a quiescent fluid at low-to-moderate Reynolds number. (a) $\Delta C_{\mathrm{D} u}^{\mathrm{W}}$; (b) $C_{\mathrm{L} u}^{\mathrm{W}}$ (with $C_{\mathrm{L} u}^{\mathrm{W}}$ multiplied by $L_{\mathrm{R}}^{-1}$ for better readability). Dashed lines: asymptotic solution (10a) and (10b) corresponding to conditions $L_{u} \ll 1, L_{\omega} \ll 1$; dotted lines: asymptotic solution (11a) and (11b) corresponding to conditions $L_{u} \gg 1, L_{\omega} \gg 1$; solid lines: semiempirical model (A1a) and (A1b).

numbers considered in this test, the numerical predictions exhibit only slight variations with the resolution, the differences between the results obtained with the coarsest $(98 \times 54 \times 64)$ grid and those provided by the most refined one being less than $1 \%$.

A second series of tests, still with the fluid at rest far from the bubble, was carried out on the $98 \times$ $54 \times 128$ grid with $0.1 \leqslant \operatorname{Re} \leqslant 500$ and $1.5 \leqslant L_{\mathrm{R}} \leqslant 8$. The drag and lift coefficients obtained in the range $0.1 \leqslant \operatorname{Re} \leqslant 2$ are compared with available analytical solutions in Fig. 21. The computational results at $\mathrm{Re}=0.1$ show good agreement with the inner solution (10) [62]. On the other hand, the results agree well with the outer solution (12) for $L_{\mathrm{R}}=4$ and 8, i.e., for $L_{u} \gtrsim O(1)$. When $L_{R} \leqslant 2$, the results deviate somewhat from both the inner and outer solutions for $\operatorname{Re} \gtrsim 0.5$. This is no surprise, as in these cases the wall lies in the intermediate region where none of these solutions is valid [63]. More precisely, the outer solution is obtained by considering that the sphere is shrunk to a point, whereas a uniformly valid approximation could be obtained only by taking into account its finite size in the Oseen equation. On a purely heuristic basis, the inner and outer solutions may be combined into expressions tending toward each of them in the appropriate limit, namely,

$$
\begin{aligned}
\Delta C_{\mathrm{D} u}^{\mathrm{W}}(\operatorname{Re} \ll 1) & \approx f_{\mathrm{D}}^{\prime} \Delta C_{\mathrm{D} u}^{\mathrm{W}-\mathrm{in}}, \\
C_{\mathrm{L} u}^{\mathrm{W}}(\operatorname{Re} \ll 1) & \approx f_{\mathrm{L}}^{\prime} C_{\mathrm{L} u}^{\mathrm{W}-\mathrm{in}}
\end{aligned}
$$

with $f_{\mathrm{D}}^{\prime}$ and $f_{\mathrm{L}}^{\prime}$ as given in (12a) and (12b), respectively. Based on these semiempirical expressions, (A1a) and (A1b) approach the inner and outer solutions in the limits $L_{u} \rightarrow 0$ and $L_{\mathrm{R}} \rightarrow \infty$ combined with $L_{u} \geqslant O(1)$, respectively. Predictions provided by these approximations are displayed with solid lines in Fig. 21 and show good agreement with the numerical results.

At moderate Reynolds number, the calculated $C_{\mathrm{L} u}^{\mathrm{W}}$ may be compared with the fit (15), under conditions $L_{\mathrm{R}} \geqslant 4$. As shown in Fig. 22, the agreement is excellent for $\mathrm{Re} \leqslant 20$, beyond which both the numerical data and the prediction indicate vanishingly small values of $C_{\mathrm{L} u}^{\mathrm{W}}$. Numerical predictions in the range $20 \leqslant \operatorname{Re} \leqslant 500$ are compared with the potential flow solution (17) at small wall distances, namely, $L_{\mathrm{R}}=1.5$ and 2.0. As seen in Fig. 22, the deviation from the irrotational solution becomes negligible beyond $\mathrm{Re} \approx 300$. At moderate $\mathrm{Re}$, the computed lift coefficient 


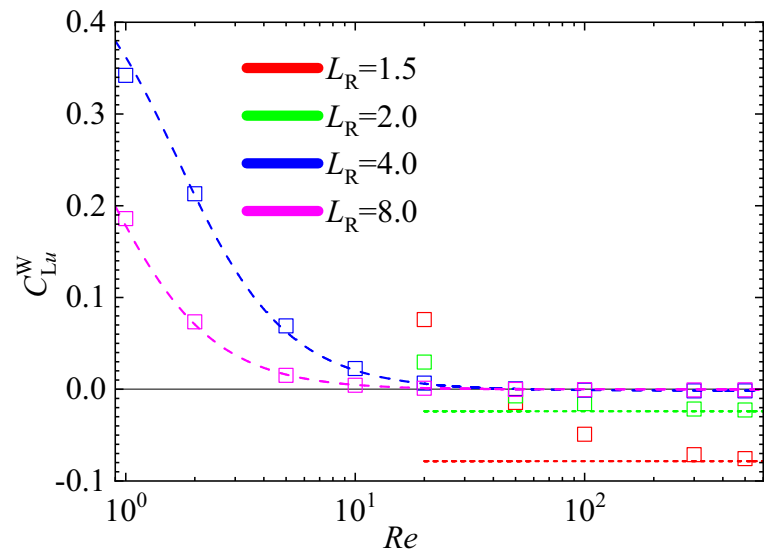

FIG. 22. Comparison of numerical predictions for $C_{\mathrm{L} u}^{\mathrm{W}}(\square)$ with the fit (15) of the asymptotic solution corresponding to conditions $L_{u} \gg 1, L_{\omega} \gg 1$ (dashed lines), and the irrotational solution (17) (short-dashed lines), for $1.5 \leqslant L_{\mathrm{R}} \leqslant 8$ and $0.1 \leqslant \operatorname{Re} \leqslant 500$.

becomes negative for $\mathrm{Re} \approx 35$ (based on linear interpolation), which agrees well with the critical value found in Refs. [6] and [9].

In a further series of tests, we computed the flow field with the $98 \times 54 \times 128$ grid in situations where the normalized wall distance $L_{\mathrm{R}}$ is very large, more specifically $\widetilde{L}=R_{\infty}$. For this purpose, the grid system was adjusted to obtain $N_{\mathrm{W}}=N_{\infty}$. The undisturbed flow is a one-dimensional linear shear flow $\boldsymbol{u}_{\infty}=\left(U_{\text {rel }}+\gamma x\right) \boldsymbol{e}_{z}$, and the forces experienced by the bubble are expected to correspond to the unbounded shear flow configuration. This test was carried out for $0.1<\operatorname{Re} \leqslant 500$ with two different shear rates, $\mathrm{Sr}=0.02$ and 0.2 . Note that these simulations differ from those carried out in Ref. [8] only with respect to the flow direction: there, the undisturbed flow was set as $\boldsymbol{u}_{\infty}=\left(U_{\text {rel }}+\right.$

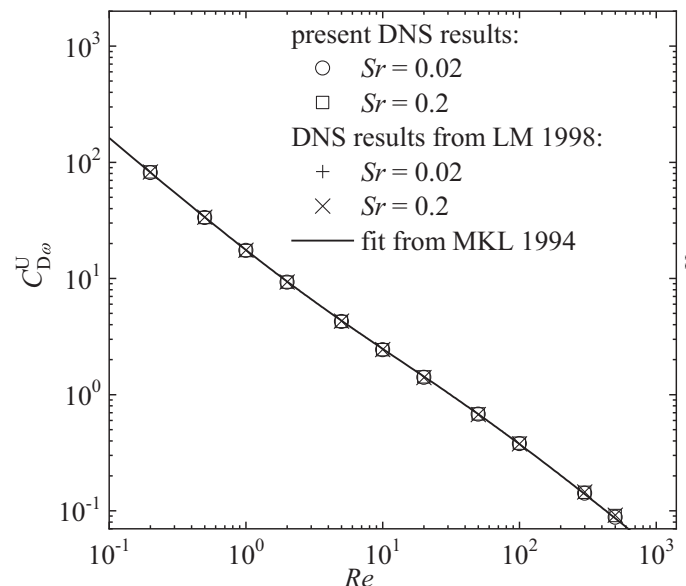

(a)

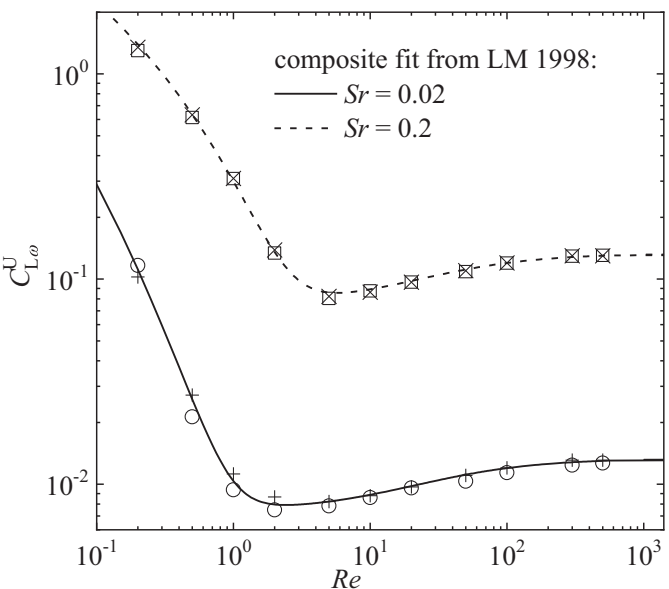

(b)

FIG. 23. Comparison of present numerical predictions with available numerical results and semiempirical expressions. (a) $C_{\mathrm{D} \omega}^{\mathrm{U}}$ compared with numerical results from Ref. [8] (LM 1998) and semiempirical correlation $\frac{\mathrm{Re}}{16} C_{\mathrm{D} 0}^{\mathrm{U}} \approx 1+\left[\frac{8}{\mathrm{Re}}+\frac{1}{2}\left(1+3.315 \mathrm{Re}^{-1 / 2}\right)\right]^{-1}$ proposed in Ref. [64] (MKL 1994). (b) $C_{\mathrm{L} \omega}^{\mathrm{U}}$ compared with numerical results and the composite fit (A2) from Ref. [8]. 
$\gamma y) \boldsymbol{e}_{x}$ (in current notations), i.e., the flow was primarily along the grid symmetry axis, whereas it stands in a plane perpendicular to this axis in the present case.

The comparison of the calculated drag coefficient with values obtained in Ref. [8] and with a semiempirical fit proposed in Ref. [64] is shown in Fig. 23(a). As the shear has virtually no effect on the drag for $\mathrm{Sr} \lesssim 0.2$, only numerical data obtained for $\mathrm{Sr}=0.2$ in Ref. [8] are presented. Results deviate from those of Ref. [8] by less than $2 \%$ for all values of Re and Sr. Figure 23(b) compares the computed lift coefficient with those obtained in Ref. [8] and with the composite fit proposed therein,

$$
C_{\mathrm{L} \omega}^{\mathrm{U}}(R e, \mathrm{Sr}) \approx \frac{S r}{|\mathrm{Sr}|}\left\{\left[C_{\mathrm{L} \omega}^{\mathrm{U}}(\operatorname{Re} \ll 1)\right]^{2}+\left[C_{\mathrm{L} \omega}^{\mathrm{U}}(\operatorname{Re} \gg 1)\right]^{2}\right\}^{1 / 2},
$$

which combines the low- and high-Re results (6b) and (9b). Again, the agreement with these earlier predictions is good. Some tiny differences may be noticed for $\mathrm{Sr}=0.02$ when $\mathrm{Re} \leqslant 2$. They are likely due to the difference in the two numerical settings. Indeed, compared with Ref. [8], the current grid is somewhat more refined $(98 \times 54 \times 128$ instead of $90 \times 45 \times 64)$, especially near the sphere surface $(2 \delta / d=0.002$ instead of $2 \delta / d=0.01)$.

In summary, the tests reported in this Appendix prove that the wall and shear effects are both accurately captured by the present numerical procedure throughout the range of Reynolds number $0.1 \leqslant \operatorname{Re} \leqslant 500$.

[1] D. Legendre, C. Colin, J. Fabre, and J. Magnaudet, Influence of gravity upon the bubble distribution in a turbulent pipe flow: Comparison between numerical simulations and experimental data, J. Chim. Phys. 96, 951 (1999).

[2] S. Subramaniam, Lagrangian-Eulerian methods for multiphase flows, Prog. Energy Combust. Sci. 39, 215 (2013).

[3] D. A. Drew, Mathematical modeling of two-phase flow, Annu. Rev. Fluid Mech. 15, 261 (1983).

[4] J. Magnaudet, S. Takagi, and D. Legendre, Drag, deformation and lateral migration of a buoyant drop moving near a wall, J. Fluid Mech. 476, 115 (2003).

[5] J. Magnaudet, A 'reciprocal' theorem for the prediction of loads on a body moving in an inhomogeneous flow at arbitrary Reynolds number, J. Fluid Mech. 689, 564 (2011).

[6] F. Takemura and J. Magnaudet, The transverse force on clean and contaminated bubbles rising near a vertical wall at moderate Reynolds number, J. Fluid Mech. 495, 235 (2003).

[7] F. Takemura, J. Magnaudet, and P. Dimitrakopoulos, Migration and deformation of bubbles rising in a wall-bounded shear flow at finite Reynolds number, J. Fluid Mech. 634, 463 (2009).

[8] D. Legendre and J. Magnaudet, The lift-force on a spherical bubble in a viscous linear shear flow, J. Fluid Mech. 368, 81 (1998).

[9] K. I. Sugioka and T. Tsukada, Direct numerical simulations of drag and lift forces acting on a spherical bubble near a plane wall, Intl. J. Multiphase Flow 71, 32 (2015).

[10] P. Shi and R. Rzehak, Lift forces on solid spherical particles in unbounded flows, Chem. Eng. Sci. 208, 115145 (2019).

[11] P. Shi and R. Rzehak, Lift forces on solid spherical particles in wall-bounded flows, Chem. Eng. Sci. 211, 115264 (2020).

[12] P. Vasseur and R. G. Cox, The lateral migration of spherical particles sedimenting in a stagnant bounded fluid, J. Fluid Mech. 80, 561 (1977).

[13] F. Takemura, S. Takagi, J. Magnaudet, and Y. Matsumoto, Drag and lift forces on a bubble rising near a vertical wall in a viscous liquid, J. Fluid Mech. 461, 277 (2002).

[14] P. G. Saffman, The lift on a small sphere in a slow shear flow, J. Fluid Mech. 22, 385 (1965).

[15] D. Legendre and J. Magnaudet, A note on the lift force on a spherical bubble or drop in a low-Reynoldsnumber shear flow, Phys. Fluids 9, 3572 (1997). 
[16] J. Magnaudet and G. Mougin, Wake instability of a fixed spheroidal bubble, J. Fluid Mech. 572, 311 (2007).

[17] L. Van Wijngaarden, Hydrodynamic interaction between gas bubbles in liquid, J. Fluid Mech. 77, 27 (1976).

[18] T. Miloh, Hydrodynamics of deformable contiguous spherical shapes in an incompressible inviscid fluid, J. Eng. Math. 11, 349 (1977).

[19] A. Biesheuvel and L. Van Wijngaarden, The motion of pairs of gas bubbles in a perfect liquid, J. Eng. Math. 16, 349 (1982).

[20] J. B. W. Kok, Dynamics of a pair of gas bubbles moving trough liquid. 1. Theory, Eur. J. Mech. B-Fluids 12, 515 (1993).

[21] F. P. Bretherton, The motion of rigid particles in a shear flow at low Reynolds number, J. Fluid Mech. 14, 284 (1962).

[22] P. G. Saffman, The lift on a small sphere in a show shear flow-Corrigendum, J. Fluid Mech. 31, 624 (1968).

[23] E. S. Asmolov, Lift force exerted on a spherical particle in a laminar boundary layer, Fluid Dyn. 24, 710 (1989).

[24] J. B. McLaughlin, Inertial migration of a small sphere in linear shear flows, J. Fluid Mech. 224, 261 (1991).

[25] T. R. Auton, The lift force on a spherical body in a rotational flow, J. Fluid Mech. 183, 199 (1987).

[26] D. A. Drew, The lift force on a small sphere in the presence of a wall, Chem. Eng. Sci. 43, 769 (1988).

[27] E. S. Asmolov, Dynamics of a spherical particle in a laminar boundary layer, Fluid Dyn. 25, 886 (1990).

[28] J. B. McLaughlin, The lift on a small sphere in wall-bounded linear shear flows, J. Fluid Mech. 246, 249 (1993).

[29] H. Lee and S. Balachandar, Drag and lift forces on a spherical particle moving on a wall in a shear flow at finite Re, J. Fluid Mech. 657, 89 (2010).

[30] V. Mathai, D. Lohse, and C. Sun, Bubbly and buoyant particle-laden turbulent flows, Annu. Rev. Condens. Matter Phys. 11, 529 (2020).

[31] J. Lu and G. Tryggvason, Numerical study of turbulent bubbly downflows in a vertical channel, Phys. Fluids 18, 103302 (2006).

[32] J. Lu, S. Biswas, and G. Tryggvason, A DNS study of laminar bubbly flows in a vertical channel, Int. J. Multiphase Flow 32, 643 (2006).

[33] J. Lu and G. Tryggvason, Effect of bubble size in turbulent bubbly downflow in a vertical channel, Chem. Eng. Sci. 62, 3008 (2007).

[34] J. Lu and G. Tryggvason, Dynamics of nearly spherical bubbles in a turbulent channel flow, J. Fluid Mech. 732, 166 (2013).

[35] A. Kanai and H. Mikata, Direct numerical simulation of wall turbulent flows with microbubbles, Intl. J. Numer. Meth. Fluids 35, 593 (2001).

[36] J. Xu, M. R. Maxey, and G. E. Karniadakis, Numerical simulation of turbulent drag reduction using micro-bubbles, J. Fluid Mech. 468, 271 (2002).

[37] J. C. Lu, A. Fernandez, and G. Tryggvason, The effect of bubbles on the wall drag in a turbulent channel flow, Phys. Fluids 17, 095102 (2005).

[38] R. F. Kunz, H. J. Gibeling, M. R. Maxey, G. Tryggvason, A. A. Fontaine, H. L. Petrie, and S. L. Ceccio, Validation of two-fluid Eulerian CFD modeling for microbubble drag reduction across a wide range of Reynolds numbers, J. Fluids Eng.-Trans. ASME 129, 66 (2007).

[39] A. Ferrante and S. E. Elghobashi, On the physical mechanisms of drag reduction in a spatially developing turbulent boundary layer laden with microbubbles, J. Fluid Mech. 503, 345 (2004).

[40] A. Ferrante and S. E. Elghobashi, Reynolds number effect on drag reduction in a microbubble-laden spatially developing turbulent boundary layer, J. Fluid Mech. 543, 93 (2005).

[41] S. Mortazavi and G. Tryggvason, A numerical study of the motion of drops in Poiseuille flow. Part 1. Lateral migration of one drop, J. Fluid Mech. 411, 325 (2000).

[42] J. Magnaudet and I. Eames, The motion of high-Reynolds-number bubbles in inhomogeneous flows, Annu. Rev. Fluid Mech. 32, 659 (2000). 
[43] D. W. Moore, The velocity of rise of distorted gas bubbles in a liquid of small viscosity, J. Fluid Mech. 23, 749 (1965).

[44] R. Adoua, D. Legendre, and J. Magnaudet, Reversal of the lift force on an oblate bubble in a weakly viscous linear shear flow, J. Fluid Mech. 628, 23 (2009).

[45] P. C. Duineveld, The rise velocity and shape of bubbles in pure water at high Reynolds number, J. Fluid Mech. 292, 325 (1995).

[46] V. G. Levich, Bubble motion at high Reynolds numbers, Zh. Eksp. Teor. Fiz. 19, 18 (1949).

[47] D. W. Moore, The boundary layer on a spherical gas bubble, J. Fluid Mech. 16, 161 (1963).

[48] D. Legendre, J. Magnaudet, and G. Mougin, Hydrodynamic interactions between two spherical bubbles rising side by side in a viscous liquid, J. Fluid Mech. 497, 133 (2003).

[49] I. Calmet and J. Magnaudet, Large-eddy simulation of high-Schmidt number mass transfer in a turbulent channel flow, Phys. Fluids 9, 438 (1997).

[50] J. Magnaudet, M. Rivero, and J. Fabre, Accelerated flows past a rigid sphere or a spherical bubble. Part 1. Steady straining flow, J. Fluid Mech. 284, 97 (1995).

[51] F. Candelier, B. Mehlig, and J. Magnaudet, Time-dependent lift and drag on a rigid body in a viscous steady linear flow, J. Fluid Mech. 864, 554 (2019).

[52] The finite-Sr correction does not appear explicitly in Ref. [8]. It was obtained by fitting the results provided in Table 6 of this reference.

[53] R. Kurose and S. Komori, Drag and lift forces on a rotating sphere in a linear shear flow, J. Fluid Mech. 384, 183 (1999).

[54] P. Bagchi and S. Balachandar, Effect of free rotation on the motion of a solid sphere in linear shear flow at moderate Re, Phys. Fluids 14, 2719 (2002).

[55] In Eq. (43a) of Ref. [4], there should be a minus sign in front of the $L_{\mathrm{R}}^{-2}$ term involved in (10b), in line with Eqs. 44(a) and 44(b). Also, the prefactor of the $L_{\mathrm{R}}^{-1} \mathrm{Sr}$ term is in error in Eq. (45b) of the same reference. All prefactors involved in (10b) were recomputed in the framework of the present study.

[56] L. F. Shampine, Vectorized adaptive quadrature in Matlab, J. Comput. Appl. Math. 211, 131 (2008).

[57] In Ref. [6], there is a misprint in Eq. (16a), which defines the fitting function $b(\mathrm{Re})$. The correct expression is that given here in (15a).

[58] G. K. Batchelor, An Introduction to Fluid Dynamics (Cambridge University Press, Cambridge, 1967).

[59] J. D. Sherwood, Steady rise of a small spherical gas bubble along the axis of a cylindrical pipe at high Reynolds number, Eur. J. Mech. B 20, 399 (2001).

[60] A. Tomiyama, H. Tamai, I. Zun, and S. Hosokawa, Transverse migration of single bubbles in simple shear flows, Chem. Eng. Sci. 57, 1849 (2002).

[61] F. Auguste and J. Magnaudet, Path oscillations and enhanced drag of light rising spheres, J. Fluid Mech. 841, 228 (2018).

[62] The slight difference observed for $L_{R}=1.5$ is presumably due to the limited accuracy of (10) when the normalized separation distance becomes of $O(1)$, as lubrication effects not accounted for by the expansion in powers of $L_{\mathrm{R}}^{-1}$ become significant in this limit.

[63] F. Takemura and J. Magnaudet, Lateral migration of a small spherical buoyant particle in a wall-bounded linear shear flow, Phys. Fluids 21, 083303 (2009).

[64] R. Mei, J. F. Klausner, and C. J. Lawrence, A note on the history force on a spherical bubble at finite Reynolds number, Phys. Fluids 6, 418 (1994). 